\title{
8.
}

\section{Allgemeine Auflösung der numerischen Gleichungen.}

(Von Herrn Dr. Encke, Professor, Director der Sternwarte, Secretair der Akademic der Wissenschaften etc. zu Berlin.)

[Diese vortreffliche Abhandlung, durch welche die Auflösung der Aufgabe, dic algebraischen Gleichungen numerisch aufzulösen, gleichsam practisch abgeschlossen wird, befindet sich in dem astronomischen Jahrbuche des Herın Verfassers für 1841 gedruckt. Da sie indessen gewils auch für diejenigen Mathematiker wichtig sein wird, welche sich nicht insbesondere mit Astronomie beschäftigen, oder welchen das genannte astronomische Jahrbuch nicht zu Gesicht kommt, so ist sie, mit Genehmigung ihres Herrn Verfassers, auch in das gegenwärtige Journal aufgenommen worden. D. H.]

Der Lösuug des Problems, welches Lagrange so ausdrückt:

Etant donnée une équation numérique sans aucune notion de la grandeur ni de la nature de ses racines, en trouver les valeurs numéques, exactes s'il est possible, ou aussi approchées qu'on voudra, ist durch Hrn. Prof. Gräffe in Zürich eine neue Seite abgewonnen worden. In seiner Schrift: Die Auflösung der höheren numerischen Gleichungen, als Beantwortung einer vou der Königl. Akad. d. Wiss. zu Berlin aufgestellten Preisfrage, Zürich 1837, zeigt er, dafs, wenn man aus einer gegebenen Gleichung eine andere ableitet, deren Wurzeln sebr hohe Potenzen der Wurzeln der gegebenen Cleichung sind, aus den Coefficienten der letzten Gleichung die reellen Wurzeln und die Moduln der imaginären sich säntlich ergeben. Err zeigt auch den einfachsten Weg zu solchen sehr hohen Potenzen der Wurzelu zu gelangen. Diese Sätze sind in den folgenden Blättern zusammengestellt, und mit dem vervollständigt, was sie noch für die gänzliche Lösung des Problems verınissen liefsen. Nämlich mit der Ermittelung der imaginären Wurzeln selhst, auf einfachem und strengem Wege; mit einer Erleichterung des Verfahrens bei Wurzeln, die nahe zusammenliegen, und auch bei sehr hohen Potenzen sich nicht entscheidend genug trennen würden, und mit den Methoden, die Werthe so weit der Wahrheit näher zu bringen, als man inmer wünschen mag.

Crelle's Journal d. M. Bd. XXII. Hft. 3. 
Die so auf Hrn. Prof. Gräffe's neuem Wege sich ergebende Auflösung empfiehlt sich in sehr hohem Grade durch ihre Allgemeinheit, Strenge und Kürze. Sie ist in so fern direct, als sie keine Versuche irgend welcher Art nöthig macht. Sie ist auf alle noch so hohen Grade der Gleichungen anwendbar, führt nie auf Gleichungen höheren Grades als die gegebene ist, und verlangt bei ilhrem stets unverändert bleibenden Verfahren nie unausführbare Rechnungen. Die Natur der Wurzeln, die Anzahl der imaginären, legt ihr durchaus kein Hindernifs in den Weg; sie giebt immer bestimmte Resultate, über deren Richtigkeit die einfachste Substitution entscheiden lärst. Sie setzt durchaus gar keine Kenntnifs von der Natur der Wurzeln voraus, so wie sie überhaupt aus den einfachsten Eigenschaften der Gleichungen sich herleiten läfst. Für die Kürze derselben spricht endlich der Umstand, dafs die Bestimmung der sämtlichen Wurzeln einer Gleichung vom $7^{\text {ten }}$ Grade bei sechs imaginären Wurzeln, so weit der Wahrheit genähert als Logarithmen von 7 Decimalen es erlauben, in etwa zwei bis drei Stunden gänzlich vollendet sein wird.

* * * *

Die Auflösung der Gleichungen kommt bekanntlich darauf hinaus: die linearen Factoren zu finden, aus deren Multiplication mit einander die Function einer Variabeln entstanden ist, welche für gewisse Werthe dieser Variabeln verschwinden soll. Etwas abweichend von dem gewöhnlichen Sprachgebrauch, werde ich die bekaunte Gröfse in einem solchen linearen Factor, die Wurzel der Gleichung nennen, so dafs wenn ein Factor einer Function von $x$ durch $x+a$ bezeichnet wird, $a$ künftig die Wurzel der Gleichung heifst, welche entsteht, wenn man die Function gleich Null setzt. Man hat bei dieser Benennung den für die numerische Rechnung angenehmen Vortheil, dals eine einfachere Betrachtung der Zeichen eintrilt. Geht man nämlich von positiven Wurzeln aus, wie es am angemessensten ist, so hat man nach dem gewöhnlichen Sprachgebrauch in einer Gleichung, die lauter positive Wurzeln hat, abwechselnde Zeiclıen, während nach der hier angenommenen Benennung lauter positive Zeichen in diesem Falle vorkommen. Der an sich unerhebliche Unterschied wird nur bemerkt, um Mifsverständnisse zu verhüten.

Betrachtet man zuerst den Fall, wo alle Wurzeln reell und unter sich verschieden sind, so ist die Gleichung entstanden aus einem Product 
von der Form

$$
(x+a)(x+b)(x+c)(x+d) \ldots=0 .
$$

Nach bekannten Lehren werden bei der wirklich ausgeführten Multiplication die Coefficienten der vcrschiedenen Potenzen von $x$ gebildet aus den Combinationen (ohne Wiederholung) der Wurzeln zu 1, zu 2, zu 3, so dafs jeder Coefficient die Summe aller älnlichen Combinationen ist. Bezeichnet man also die Summen solcher Combinationen, je nach dem Grade derselben, mit $[a],[a b],[a b c]$ etc., so wird die entwickelte Gleichung

$$
x^{n}+[a] x^{n-1}+[a b] x^{n-2}+[a b c] x^{n-3}+[a b c d] x^{n-4} \ldots=0 .
$$

Aus den Coefficienten einer solchen Gleichung kann man aber nach bekannten Lehren alle symmetrischen Functionen der $\mathbf{W}$ urzeln finden und numerisch berechnen, ohne die $\mathbf{W}$ urzeln selbst zu kennen. Man kann folglich auch vermittelst dieser Coefficienten die Combinationen beliebig hoher Potenzen der Wurzeln zu 1, zu 2, zu 3 bestimmen, oder die Summen $\left[a^{m}\right]$, $\left[a^{m} b^{m}\right],\left[a^{m} b^{m} c^{m}\right]$ etc. Folglich kann man auch die sämtlichen Coefficienten einer Gleichung angeben, deren Wurzeln die $\boldsymbol{m}^{\text {ten }}$ Potenzen der Wurzeln der ursprünglich gegebenen Gleichung sind, nämlich

$$
\boldsymbol{x}^{n}+\left[\boldsymbol{a}^{m}\right] x^{n-1}+\left[\boldsymbol{a}^{m} \boldsymbol{b}^{m}\right] \boldsymbol{x}^{n-2}+\left[\boldsymbol{a}^{m} \boldsymbol{b}^{m} \boldsymbol{c}^{m}\right] \boldsymbol{x}^{n-3}+\ldots .=0 .
$$

Die Grö[se von $m$ kann ganz beliebig, so hoch man will, angenommen werden. Man nehme nun an, um den Gang der Entwickelung leichter zu übersehen, es sei unter den Wurzeln $a$ die grörste, $b$ die nächst grörste, $c$ die folgende etc. oder es sei

$$
a>b, \quad b>c, \quad c>d, \quad d>e \ldots . \text { etc., }
$$

ferner sei $m$ eine sehr hohe Potenz, so wird in

$$
\left[a^{m}\right]=a^{m}+b^{m}+c^{m}+d^{m}+e^{m} \ldots .
$$

für einen gewissen Grad der Näherung, endlich einmal der Fall stattfinden, bei stets vergröfsertem $m$, dafs $e^{m}$ verschwindet oder vernachlässigt werden kann gegen $d^{m}, d^{m}$ gegen $c^{m}$, $c^{m}$ gegen $b^{m}, b^{m}$ gegen $a^{m}$, und also auch die Summe aller Potenzen der kleineren Wurzeln gegen die Potenz der gröfsten. In diesen Falle wird man setzen können

$$
\left[a^{m}\right]=a^{m} \text {. }
$$

Das ähnliche wird in der Summe $\left[a^{m} b^{m}\right]$ stattfinden in Bezug auf das Glied $a^{m} b^{m}$, welches zuletzt nothwendig gegen die Summe aller andern $a^{m} c^{m}$, $a^{m} d^{m}, b^{m} c^{m}$ etc. überwiegen mufs. Es wird folglich ebenfalls dann gesetzt werden können

$$
\left[a^{m} b^{m}\right]=a^{m} b^{m}
$$


und ganz analog bei allen folgenden Glielern, oder die Endgleichung wird bei stets vergröfsertem $m$ endlich einmal die Form anuehmen

$$
x^{n}+a^{m} x^{n-1}+a^{m} b^{m} x^{n-2}+a^{m} b^{m} c^{m} x^{n-3}+a^{m} b^{m} c^{m} d^{m} x^{n-4}+\ldots=0 .
$$

Hat man die Coefficienten dieser Gleichung numerisch, so hat man unmittelbar $a^{m}$; durch Division von $\frac{a^{m} b^{m}}{a^{m}}$ dann auch $b^{m}$; nachher aus dem Bruche $\frac{a^{m} b^{m} c^{m}}{a^{m} b^{m}}$ ebenfalls $c^{m}$, und überhaupt die $m^{\text {ten }}$ Potenzen aller Wurzeln zu gleicher Zeit, aus denen sich die Wurzeln selbst durch Ausziehung der $\boldsymbol{m}^{\text {ten }}$ Wurzel ergeben. Das Kennzeichen, ob für einen bestimmten Grad der Näherung die Grenze erreicht sei, wird man darin finden, dafs wenn man von der Potenz $m$, zu der Potenz $m^{\prime}$ übergeht, also aus der obigen Gleichung die folgende bildet

$$
x^{n}+\left[a^{m^{\prime}}\right] x^{n-1}+\left[\boldsymbol{a}^{m^{\prime}} \boldsymbol{b}^{m^{\prime}}\right] \boldsymbol{x}^{n-2}+\left[\boldsymbol{a}^{m^{\prime}} \boldsymbol{b}^{m^{\prime}} \boldsymbol{c}^{m^{\prime}}\right] \boldsymbol{x}^{n-3}+\ldots=0,
$$

die Coefficienten der gleichen Potenzen von $x$ in beiden Gleichungen sich verhalten wie die $m^{\text {e }}$ Potenz einer Gröfse zu der $m^{\text {ten }}$ derselben, oder wenn man die Logarithmen eines Coefficienten von $x^{n-r}$ in beiden Gleichungen hat, die etwa durch $\lg \alpha_{\iota_{n}}$ in der ersten, $\lg \alpha_{m}$ in der zweiten bezeichuet werden mögen, so unufs für den angenommenen Grad der Näherung

$$
\frac{1}{m} \lg \alpha_{m}=\frac{1}{m^{\prime}} \lg \alpha_{m^{\prime}} \quad \text { oder } \quad \lg \alpha_{m^{\prime}}=\frac{m^{\prime}}{m} \lg \alpha_{m}
$$

sein, und zwar bleibend, da in speciellen Fällen es wohl sein kann, dafs die Summe sämtlicher kleinerer Wurzeln und ihrer Combinationen doch noch erheblich genug ist, um ein ähnliches Verhältnifs hervorzurufen. Indessen wird die Möglichkeit dieser Ausuahme immer verringert werden, je melır $m$ wächst, und wird zuletzt ganz aufhören.

Wollte man die Erhebung zu solchen sehr hohen Potenzen auf die gewöhnliche Art durch Bildung der symmetrischen Functionen bewirken, so würde die Rechuung nicht ausführbar seill. Man erreicht aber dasselbe, wenn man stufenweise erst die Wurzeln zur Potenz $p$ erhebt, und die Gleichung bildet, welche den $a^{p}, b^{p}$ etc. entspricht. Leitet man aus den numerisch berechneten Coefficienten dieser Gleichung die andere ab, welche die $p^{\text {te }}$ Potenz der Wurzeln derselben enthält, so hat man die Gleichung, deren Wurzeln die $\boldsymbol{p} \boldsymbol{p}^{\text {te }}$ Potenz der Wurzeln der ursprünglich gegebenen Gleichung sind, und fährt man so fort, so erhält man nach und nach Gleichungen, deren Wurzeln

$$
a^{p} \quad a^{p^{3}} \quad a^{p^{3}} \text { etc. }
$$


8. Encke, ̈̈ber allgemeine Auflösung der numerisehen Gleichungen.

sind, wo folglich $m$ gleich einer Potenz von $p$ sehr schnell wächst. Schon die kleinsten Zahlen für $\boldsymbol{p}$ werden hier alle Bequemlichkeit gewähren.

Wäre zuerst $\boldsymbol{p}=2$ und die vorgegebene Gleichung:

$$
x^{n}+\alpha_{1} x^{n-1}+\alpha_{2} x^{n-2}+\alpha_{3} x^{n-3} \ldots+\alpha_{n}=0 \text {, }
$$

so schreibe man für $x \ldots \ldots x^{\frac{1}{2}}$. Die linearen Factoren dieser Gleichungen werden dann sein

$$
\left(x^{\frac{1}{2}}+a\right)\left(x^{\frac{1}{2}}+b\right)\left(x^{\frac{1}{2}}+c\right) \ldots=0 .
$$

Schafft man aus ihr alle Wurzelgrölsen weg, so werden die Factoren der neuen Gleichung

$$
\left(x-a^{2}\right)\left(x-b^{2}\right)\left(x-c^{2}\right) \ldots=0,
$$

und um positive Wurzeln zu erhalten (in dem obigen Sinne), ändere man das Zeichen aller Glieder, die einer ungeraden Potenz angehören, oder überhaupt, der zweiten, vierten etc., d. h. einer geraden Ordnungszahl angehörigen, wenn man von der höchsten Potenz von $x$ anfängt und keine übergeht.

Zur Wegschaffung der Wurzelgröfsen kann man davon ausgehen, dafs für $p+q=0$ auch $p^{2}-q^{2}=0$. Wenn man die Gleichung also in zwei solche Theile abtheilt, dals jeder für sich, wenn man ihn in das Quadrat erhebt, von aller Irrationalität frei ist, so ist das Verlangte erreicht. Diese Theile können in jeden Falle sein

und

$$
x^{\frac{n}{2}}+\alpha_{2} x^{\frac{n-2}{2}}+a_{4} x^{\frac{n-4}{2}}+a_{6} x^{\frac{n-6}{2}}
$$

und

$$
a_{1} x^{\frac{n-1}{2}}+a_{3} x^{\frac{n-3}{2}}+a_{5} x^{\frac{n-5}{2}}+a_{7} x^{\frac{n-7}{2}} \text {. }
$$

Ihre Quadrate sind

$$
\left.\left.\begin{array}{rl}
x^{n}+2 \alpha_{2} x^{n-1}+\alpha_{2}^{2} & x^{n-2}+2 \alpha_{2} \alpha_{4} \\
+2 \alpha_{4}
\end{array}\right\} \begin{array}{rl}
x^{n-3} & +\alpha_{4}^{2} \\
& +2 \alpha_{16} \\
& +2 \alpha_{2} \alpha_{6} \\
& +2 a_{8}
\end{array}\right\}
$$

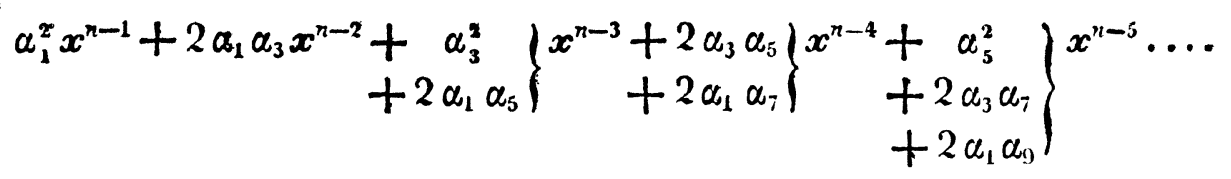

Nimmt man die Differenz dieser Quadrate und ändert die Zeichen wie eben bemerkt, so wird

$$
\left.\left.\left.\left.\begin{array}{c}
x^{n}+\alpha_{1}^{2} \\
-2 \alpha_{2}
\end{array}\right\} \begin{array}{c|c}
x^{n-1}+\alpha_{2}^{2} \\
-2 a_{1} \alpha_{3} \\
+2 \alpha_{4}
\end{array}\right\} \begin{array}{r}
x^{n-2}+\alpha_{3}^{2} \\
-2 \alpha_{2} \alpha_{4} \\
+2 \alpha_{1} \alpha_{5} \\
-2 \alpha_{6}
\end{array}\right\} \begin{array}{r}
x^{n-3}+\alpha_{4}^{2} \\
-2 \alpha_{3} \alpha_{5} \\
+2 \alpha_{2} \alpha_{6} \\
-2 \alpha_{1} \alpha_{7} \\
+2 \alpha_{3}
\end{array}\right\}
$$


die Gleichung sein, deren Wurzeln $a^{2}, b^{2}, c^{2}$ etc. sind. Diese Form giebt eine höchst einfache und übersichtliche Rechnung. Der Coefficient einer Potenz von $x$ in der neuen Gleichung, wird gebildet durch die Verbindung des Quadrats des Coefficienten derselben Potenz in der schon berechueten Gleichung, mit den doppelten Producten je zweier gleich weit zu beiden Seiten von ihm abstehender Coefficienten, die letzteren regelmäfsig mit abwechselnden Zeichen genommen.

Eine ähnliche Ableitung kann man auch für $p=3$ machen. Da jedesmal, was auch $p, q$ und $r$ sein mögen,

$$
(p+q+r)^{3}=p^{3}+q^{3}+r^{3}+3(p+q+r)(p q+q r+p r)-3 p q r
$$

ist, so wird auch immer, wenn $p+q+r=0$,

$$
p^{3}+q^{3}+r^{3}-3 p q r=0 .
$$

Wenn man also in der gegebenen Gleichung statt $x \ldots x^{\frac{1}{3}}$ schreibt, wodurch die linearen Factoren werden $x^{\frac{1}{3}}+a, x^{\frac{1}{3}}+b$ und hier die Wurzelgröfsen wegschaffit, so erhält man die Factoren $x+a^{3}, x+b^{3}, x+c^{3}$, ohne dafs es nöthig wäre, die Zeichen nachher noch zu ändern. Zu dieser Wegschaffung ist es nach der eben angeführten Gleichung nur erforderlich, die Gleichung in drei solche Theile zu theilen, dafs der Cubus jedes einzelnen und das Product aller drei frei von einer Irrationalität ist. Solche Theile können immer sein:

$$
\begin{aligned}
& x^{\frac{n}{3}}+\alpha_{3} x^{\frac{n-3}{3}}+\alpha_{6} x^{\frac{n-6}{3}}+\ldots=A \\
& \alpha_{1} x^{\frac{n-1}{3}}+\alpha_{4} x^{\frac{n-4}{3}}+\alpha_{7} x^{\frac{n-7}{3}} \ldots \ldots=\boldsymbol{B} \\
& \alpha_{2} x^{\frac{n-2}{3}}+\alpha_{5} x^{\frac{n-5}{3}}+\alpha_{8} x^{\frac{n-8}{3}} \ldots \ldots=C \text {. }
\end{aligned}
$$

Denn sie werden

$$
\begin{aligned}
& x^{\frac{n}{3}}\left\{1+\alpha_{3} x^{-1}+\alpha_{6} x^{-2} \ldots .\right\}=\boldsymbol{A} \\
& x^{\frac{n-1}{3}}\left\{\alpha_{1}+\alpha_{4} x^{-1}+\alpha_{7} x^{-2} \ldots .\right\}=\boldsymbol{B} \\
& x^{\frac{n-2}{3}}\left\{\alpha_{2}+\alpha_{5} x^{-1}+\alpha_{8} x^{-2} \ldots .\right\}=\boldsymbol{C},
\end{aligned}
$$

die offenbar, jeder für sich zum Cubus erhoben, und mit einander multiplicirt, frei von einer Irrationalität sind. Bildet man also

$$
A^{3}+B^{3}+C^{3}-3 A B C
$$

so werden die ersten Glieder 


$$
\begin{aligned}
& x^{n}+\left(a_{1}^{3}-3 a_{1} \alpha_{2}+3 \alpha_{3}\right) x^{n-1} \\
& +\left(a_{2}^{3}+3 a_{1}^{2} a_{4}-3 a_{1} \alpha_{5}-3 \alpha_{1} \alpha_{2} \alpha_{3}-3 a_{2} a_{7}+3 a_{3}^{2}+3 a_{6}\right) x^{n-2} \\
& +\left\{\begin{array}{c}
\alpha_{3}^{3}-3 \alpha_{1} \alpha_{8}+3 \alpha_{1}^{2} \alpha_{7}-3 \alpha_{1} \alpha_{2} \alpha_{6}-3 \alpha_{1} \alpha_{3} \alpha_{5}+3 \alpha_{1} \alpha_{4}^{2}+3 \alpha_{2}^{2} \alpha_{5} \\
-3 \alpha_{2} \alpha_{3} \alpha_{4}-3 \alpha_{2} \alpha_{7}-3 \alpha_{4} \alpha_{5}+6 \alpha_{3} \alpha_{6}+3 \alpha_{9}
\end{array}\right\} x^{n-3} \text {. }
\end{aligned}
$$

Der Vortheil der Kürze und Einfachheit ist so entschieden bei dem Falle $\boldsymbol{p}=2$, dafs weder das bedeutend langsamere Fortschreiten der Potenzen 2, 4, 8, 16 etc., verglichen mit 3, 9, 27 etc., ihm Eintrag thut, noch selbst der Unstand, dafs für $\boldsymbol{p}$ gleich einer geraden Zahl, der Unterschied zwischen einer positiven und einer negativen Wurzel gleich anfangs verschwindet, während eine ungerade Potenz ihn bestehen läfst. Wenn man die Wurzel ihrer absoluten Gröfse nach kennt, und nur das Zeichen ungewils ist, so reicht eine einfache Substitution, wobei man die geraden und ungeraden Potenzen von $x$ von einander trennt, sogleich hin, un darüber zu entscheiden. Sonst könnte man auch durch Substitution der nächsten positiven und negativen Grenzen in runden Zahlen um so unbedenklicher darüber sich versichern, als man alle andern Wurzeln gleichzeitig kennen lernt, und folglich die Grenzen stets so nehmen kann, dafs nur die eine Wurzel innerhalb derselben vorhanden ist.

Eine solche Substitution des zuerst gefundenen Werthes wird man doch nicht vermeiden köunen, abgesehen von der Prüfung der Richtigkeit, die sie gewährt, da es niemals rathsam sein wird, gleich anfangs die Grenze der Genauigkeit, bis zu welcher man gehen will, mil einemmale zu umfassen. Die Rechnung mufs mit Logarithmen ausgeführt werden. Aus einem später zu erwähnenden Grunde sind Logarithmen von fünf Decimalen, in jedem Falle, wo man eine grofse Genauigkeit haben will, vorzuziehen. Angenommen daher, was später immer vorausgesetzt werden soll, es werde die erste Rechnung mit Logarithmen von fünf Decimalen und so ausgeführt, dafs man nach Potenzen von 2 fortschreitet, so kann man sowohl im Voraus übersehen, wie weit man gehen, wie viele solcher Rechnungen man machen mufs, als auch das Verfahren, wie der gefundene Werth am bequemsten verbessert wird, angeben.

Die Grenze für alle Wurzeln wird erreicht sein, wenn das Quadrat jedes Coefficienten, so gegen das doppelte Product der ihm zur Seite stehenden überwiegt, dafs das letztere auf die fünfte Decimale keinen Einflufs mehr hat, oder bei Logarithmen von fünf Decimalen kleiner als der $100,000^{\text {te }}$ Theil des ersteren ist. Das gröfste Product, wenn man sich der 
Grenze einmal schon genähert hat, werden immer die beiden nächsten Coefficienten geben. Denn wenn die Reihefolge der Coefficienten

$$
\boldsymbol{a}^{m} \boldsymbol{b}^{m}, \quad \boldsymbol{a}^{m} \boldsymbol{b}^{m} \boldsymbol{c}^{m}, \quad \boldsymbol{a}^{m} \boldsymbol{b}^{m} \boldsymbol{c}^{m} d^{m}, \quad \boldsymbol{a}^{m} \boldsymbol{b}^{m} c^{m} d^{m} e^{m}, \quad \boldsymbol{a}^{m} \boldsymbol{b}^{m} \boldsymbol{c}^{m} d^{m} e^{m} f^{m},
$$

ist, so wird für den mittelsten der Werth in der neuen Gleichnng werden:

$$
a^{2 m} b^{2 m} c^{2 m} d^{2 m}-2 a^{2 m} b^{2 m} c^{2 m} d^{m} e^{m}+2 a^{2 m} b^{2 m} c^{m} d^{m} e^{m} f^{m}
$$

wo das zweite Glied zum dritten sich verhält wie $c^{m}: f^{m}$, dagegen das erste zum zweiten wie $d^{\prime n}: 2 e^{m}$. Ueberhaupt kommit man sehr bald dahin, dafs die folgenden Glieder nach dem zweiten unbeträchtlich werden. Soll aber das zweite gegen das erste verschwinden, so dafs es nicht mehr in Rechnung gebracht werden kann, so mufs

d. $\mathbf{h}$.

$$
100,000 \cdot e^{m}<\frac{1}{2} d^{m} \text { oder }\left(\frac{d}{e}\right)^{m}>200,000
$$

$$
m>\frac{5,30103}{\lg \frac{d}{e}}
$$

Hieraus ergiebt sich für die Werthe

$$
\begin{array}{llr}
\frac{d}{e}=1,1 & m=128=2^{7} \\
\frac{d}{e}=1,01 & m=1227<2^{11} \\
\frac{d}{e}=1,001 & m=12215<2^{14}
\end{array}
$$

und bei einem grölseren $\frac{d}{e}$ natürlich eine um so viel geringere Auzahl vou Operationen. Es folgt hieraus, dafs man in der Regel mit sieben Umformungen völlig ausreicht. In dem ungewöhnlichen Falle von so äufserst nahe liegenden Wurzeln, wie für $\frac{d}{c}=1,01$ oder 1,001, würde man doch nur elf und vierzehn Operationen gebrauchen. Allein es wird später gezeigt werden, dafs Fälle solcher selır nahe liegenden $W$ urzeln, nach der Art der gleichen Wurzeln behandelt werden können, so dafs man die Oporationen gar nicht nöthig hat so weit fortzusetzen, bis die Wurzeln selbst von einander getreunt sind, sondern nur so weit, bis ihr Product sich von den Producten der übrigen Wurzehn mit einander unterscheidet. Bei hänfigen Anwendungen ist mir kein Beispiel vorgekommen, wo, auch im ungünstigsten Falle von sieben Wurzelı, die sämtlich zwischen 1,1 und 1,6 lagen, mehr als acht Operationen nöthig gewesen wären. 
8. Enke, ̈̈ber allgemeine Auflösung der numerischen Gieichungen.

In der Regel wird man bei einer Rechnung mit fünf Decimalen, nach Ausziehung der $m^{\text {ten }}$ Wurzel, den Werth der Wurzel sehr genau erhalten, so dafs die fünfte Decimale immer sicher, und meistentheils selbst die sechste es ist. Dieses scheint daher zu kommen, dafs im Anfange, bei den ersten Operationen, die Coefficienten sich aus mehreren Theilen zusammensetzen, so dafs die Ungewifsheit der letzten Stelle verringert wird, weil selten alle Fehler der letzten Decimale auf eine Seite fallen. Bei der Ausziehung der $\boldsymbol{m}^{\text {ten }}$ Wurzel dividirt man aber auf einmal mit einer grofsen Zahl, und vermindert so die Ungewifsheit der letzten Decimale.

Bei dieser sehr grofsen Aunäherung an die Wahrheit, bis auf den 100,000 ${ }^{\text {sten }}$ Theil des Ganzen, kann man unbedenklich den Taylorschen Lehrsatz oder die Newtonsche Approximationsmethode anwenden; denn die Unsicherheit derselben findet nur dann statt, wenn der Werth, von dem man ausgeht, nicht blofs einer, sondern mehreren Wurzeln selir nahe ist. Nach dem Taylorschen Satze wird für

$$
\begin{gathered}
x=x_{0}+\Delta x_{0} \\
f x=f x_{0}+\frac{d f x_{0}}{d x_{0}} \Delta x_{v}+\ldots
\end{gathered}
$$

Hat $f x$ die Form, in der die Gleichungen immer als gegeben angesehen werden

$$
x_{0}^{n}+a_{1} x_{0}^{n-1}+a_{2} x_{0}^{n-2},
$$

so wird $\frac{d f x_{0}}{d x_{0}}=n x_{0}^{n-1}+(n-1) \alpha_{1} x_{0}^{n-2}+(n-2) a_{2} x_{1}^{n-3}+\ldots$

oder $\quad x_{0} \frac{d f x_{0}}{d x_{0}}=n x_{0}^{n}+(n-1) a_{1} x_{0}^{n-1}+(n-2) a_{2} x_{11}^{n-2}+\ldots$

Hat man also die Substitution von $x_{0}$, dem gefundenen genäherten Werthe, in die Gleichung gemacht, wovon das Resultat mit $\left[x_{0}^{n}\right]$ bezeichnet werden möge, so multiplicirt man jedes Glied mit den Exponenten der Potenz von $x$, die darin vorkommt; das Resultat dieser Operation möge mit $\left[n x_{0}^{n}\right]$ bezeichnet werden; dann wird

$$
\frac{\Delta x_{0}}{x_{0}}=\Delta \lg x_{0}=-\frac{\left[x_{0}^{n}\right]}{\left[n x_{0}^{n}\right]} M,
$$

wo $\boldsymbol{M}$ der Modulus des briggischen Systems ist, dessen logar. $=9,637 \% 843$. Auf diese Weise wird man ohne eiıa gröfsere Mühe, als die Substitution des Werthes von $x_{0}$ in die Gleichung, den Werth von $\lg x$ so genau erhalten, als Logarithmen von 7 Decimalen ilı zu geben vermögen, da die Multiplication mit dèn Exponenten kaum in Betracht kommt. Eine gröfsere 
8. Encke, über allgemeine Auflösung der numerischen Gleichungen:

Genauigkeit wird kaum je verlangt werden, und kann, wenn sie gewünscht wird, auf dieselbe Weise erhalten werden.

Betrachtet man zweitens den Fall, in welchem alle Wurzeln imaginär sind, und unter diesen wiederum keine mit der andern zusammenfallend, so wird es, um mit imaginären Gröfsen nicht in der Rechnung zu thun zu haben, am gerathensten sein, von den trinomischen Factoren auszugehen, in welche sich jede solcho Gleichung zerlegen lassen muls. Sei die allgemeine Form eines solchen Factors

$$
x^{2}+f x+g^{2}
$$

wo $g$ bei imaginären Wurzeln stets reell ist, so sind die beiden linearen Factoren dieser Gröfse hekanntlich von der Form

oder auch, wenn

$$
x+\alpha+\beta \sqrt{ }-1 \text { und } x+\alpha-\beta \sqrt{ }-1
$$

$$
\begin{aligned}
& \frac{\alpha}{\sqrt{\left(\alpha^{2}+\beta^{2}\right)}}=\cos \varphi, \quad \frac{\beta}{\sqrt{\left(\alpha^{2}+\beta^{2}\right)}}=\sin \varphi, \\
& x+g(\cos \varphi+\sin \varphi r-1) \text { und } x+g(\cos \phi-\sin \varphi r-1)
\end{aligned}
$$
so dafs
$\boldsymbol{g}=\boldsymbol{r}\left(\alpha^{2}+\beta^{2}\right)$
und
$f=2 g \cos \varphi$,

folglich für imaginäre Wurzeln oder bei reellem $\varphi$ stets

$$
f<2 g \text {. }
$$

Werden aus solchen Factoren die Factoren hergeleitet, welche die $\mathbf{m}^{\text {ten }}$ Potenzen der Wurzeln enthalten, so werden diese letzteren, wegen

vollständig werden :

$$
(\cos \varphi \pm \sin \varphi r-1)^{m}=\cos m \varphi \pm \sin m \phi \checkmark-1
$$

$$
x+g^{m}(\cos m \varphi+\sin m \varphi r-1), \quad x+g^{m}(\cos m \varphi-\sin m \varphi r-1),
$$

woraus der trinomische Factor entsteht

$$
x^{2}+2 g^{m} \cos m \varphi x+g^{2 m}
$$

oder wenn man ihn bezeichnet durch

$$
x^{2}+f_{1 n} x+g^{2 m}
$$

so wird wiederum bei imaginären Wurzeln

$$
f_{m}=2 g^{m} \cos m \varphi \leqq 2 g^{m},
$$

d. h. $f_{m}$ kann nie gröfser als $2 g^{m}$ werden, abgesehen vom Zeichen. Es kann nämlich $f_{m}=2 g^{m}$ werden, wenn $m \varphi$ ein Vielfaches von $\pi$ ist, und wenn es dieses einmal geworden ist, so wird es bei der Erhebung in das Quadrat oder die höheren Potenzen stets diesen Werth behalten. Imaginäre Wurzeln geben in diesem speciellen Falle dasselbe Resultat, wie gleiche reelle. In allen andern Fällen aber wird $f_{m}$, je nach dem ver- 
schiedenen Werthe von $\cos m \varphi$, bald ab-, bald zunehmen, im Zeichen wechseln, stets aber der absoluten Gröfse nach kleiner als $2 g^{m}$ bleiben.

Hat nun eine Gleichung lauter imaginäre Wurzeln, so wird sie das Product lauter solcher trinomischer Factoren sein, in welchen $f<2 g$ ist. Die Form dieses Productes wird, wenn die einzelnen Factoren sind,

$$
x^{2}+f x+g^{2}, \quad x^{2}+f^{\prime} x+g^{\prime 2}, \quad x^{2}+f^{\prime \prime} x+g^{\prime \prime 2} \ldots . . \text { etc. },
$$
und, wenn die obige Summen-Bezeichnung beibehalten wird,

$$
\begin{aligned}
x^{2 n}+[f] x^{2 n-1} & +\left(\left[g^{2}\right]+\left[f f^{\prime}\right]\right) x^{2 n-2} \\
& +\left(\left[g^{2} f^{\prime}\right]+\left[f f^{\prime} f^{\prime \prime}\right]\right) x^{2 n-3} \\
& +\left(\left[g^{2} g^{\prime 2}\right]+\left[g^{2} f^{\prime} f^{\prime \prime}\right]+\left[f f^{\prime} f^{\prime \prime} f^{\prime \prime \prime}\right]\right) x^{2 n-4} \\
& \vdots \\
& +\left(\left[g^{2} g^{\prime 2} \ldots g^{(n-2)^{2}}\right]+\left[g^{2} g^{\prime 2} \ldots g^{(n-3)^{2}} f^{(n-2)} f^{(n-1)}\right]\right) x^{2} \\
& +\left[g^{2} g^{\prime 2} \ldots g^{(n-2) 2} f^{(n-1)}\right] x \\
& +g^{2} g^{\prime 2} \ldots . g^{(n-1)^{2}}
\end{aligned}
$$

Dafs diese Form die richtige ist, wird man aus den einfachen Gesetzen der Multiplication ableiten können, und dafs namentlich in den letzten Gliedern die $f$ einzeln, oder zu zweien, oder zu dreien mit dem $g$ combinirt vorkommen müssen, eben so wie in den ersten Gliedern, so dafs das zweite und vorletzte, das dritte und drittletzte etc. einander in Bezug auf den Grad der $f$, die mit einander und den $g$ multiplicirt sind, entsprechen, wird man sogleich übersehen, wenn man die trinomischen Factoren so schreibt

$$
g^{2}\left\{1+\frac{f}{g^{2}} x+\frac{1}{g^{2}} x^{2}\right\}, \quad g^{\prime 2}\left\{1+\frac{f^{\prime}}{g^{\prime 2}} x+\frac{1}{g^{\prime 2}} x^{2}\right\} \quad \text { etc. }
$$

und sie dann mit einander multiplicirt.

Werden nun aus einer solchen Gleichung die Gleichungen hergeleitet, deren Wurzeln die $m^{\text {ten }}$ Potenzen der ursprünglichen Wurzeln sind, so geht $f$ über in $f_{m}, f^{\prime}$ in $f_{m}^{\prime}$ etc., $g^{2}$ in $g^{2 m}, g^{\prime 2}$ in $g^{2^{2 m}}$ und die neue Gleichung wird folglich die Form haben:

$$
\begin{aligned}
x^{2 n}+\left[f_{m}\right] x^{2 n-1} & +\left(\left[g^{2 m}\right]+\left[f_{m} f_{m}^{\prime}\right]\right) x^{2 n-2} \\
& +\left(\left[g^{2 m} f_{m}^{\prime}\right]+\left[f_{m} f_{m}^{\prime} f_{m}^{\prime \prime}\right]\right) x^{2 n-3} \\
& +\left(\left[g^{2 m} g^{2 m}\right]+\left[g^{2 m} f_{m}^{\prime} f_{m}^{\prime \prime}\right]+\left[f_{m} f_{m}^{\prime} f_{m}^{\prime \prime} f_{m}^{\prime \prime \prime}\right]\right) x^{2 n-4} \\
\vdots & \vdots \\
& +\left(\left[g^{2 m} g^{2 m} \ldots g^{(n-2)^{2 m}}\right]+\left[g^{2 m} g^{2 m} \ldots g^{(n-3)^{2 m}} f_{m}^{n-2} f_{m}^{n-1}\right]\right) x^{2} \\
& +\left[g^{2 m} g^{\prime 2 m} \ldots . g^{(n-2)^{2 m}} f_{m}^{n-1}\right] x \\
& +g^{2 m} g^{{ }^{2 m}} \ldots . g^{(n-1)^{2 m}}
\end{aligned}
$$


Nimmt man hier wiederum zur leichteri Uebersicht des Ganges an, dafs $g$ der grölste Modul (nach der gewölmlichen Benennung), $g^{\prime}$ der nächstgröfste, $g^{\prime \prime}$ der folgende u. s. w., oder dals

$$
g>g^{\prime}, \quad g^{\prime}>g^{\prime \prime}, \quad g^{\prime \prime}>g^{\prime \prime \prime} \ldots
$$

und kein Modul dem andern gleich ist, und betrachtet man zuerst die Glieder, in welchen die Potenz eine gerade $\mathbf{Z}$ ahl ist, so wird bei ihren Coefficienten das jedesmal vorkommende, von allen $f_{m}$ ganz freie Glied .... $\left[g^{2 m} g^{\prime 2 m} g^{\prime 2 m} \ldots.\right]$, ganz ähnlich wie bei den reellen Wurzeln, bei vergröfsertem $m$ zuletzt übergehen in

$$
\boldsymbol{g}^{2 m} \boldsymbol{g}^{2 m} \boldsymbol{g}^{\prime 2 m} \ldots \text {. }
$$

so dals für $\left[g^{2 m}\right]$ geschrieben werden kann $g^{2 m}$, für $\left[g^{2 m} g^{2^{2 m}}\right] \ldots g^{2 m} g^{\prime 2 m}$ u. s. w. Neben diesen Summen kommen aber noch theils solche Summen vor, in denen melrere $g$ mit mehreren $f$ verbunden sind, oder auch solche, wo nur $f$ darin enthalten sind. In allen diesen Summen müssen die $f$ immer in gerader Zahl vorhanden sein. Substituirt man hier für jedes $f_{m}$ seine äufserste Grenze $2 g^{m}$, so wird das Resultat zuverlässig immer gröfser oder gleich grofs mit dem eigentlichen Werthe, und die Grenze, der sich mit vergröfsertem $m$ diese Summen näheru, wenn man in ihnen jedes $f_{m}$ mit $2 g^{m}$ vertauscht hat, kann nie überschritten werden. Hiernach wird bei dem Coefficienten von $x^{2 n-2}$ die Summe $\left[f_{m} f_{m}^{\prime}\right]$ niemals die Summe von $4\left[g_{m} g^{\prime m}\right]$ überschreiten können, folglich wird auch die Grenze dieser letzten Summe bei vergröfsertem $m$, nämlich die Gröfse $4 g^{m} g^{\prime^{m}}$ selbst der äufserste Grenzwerth für $\left[f_{m} f_{m}^{\prime}\right]$ sein. Von den beiden Theilen aber, aus denen der Coefficient zuletzt allein besteht,

$$
g^{2 m}+4 g^{m} g^{\prime^{m}}
$$

wird auch der zweite zuletzt verschwinden müssen gegen den ersten, sobald

d. h. sobald

$$
g^{m}>4 g^{\prime m}
$$

$$
g>g^{\prime} \stackrel{m}{\vee} 4,
$$

denn in einem solchen Falle wird irgend einmal, wenn $m$ erhöht worden ist zu $m^{p}, g^{m^{p}}$ ganz und gar überwiegen. Die Zahl 4 unter dem Wurzelzeichen ist unabhängig von der Potenz $m$, und bleibt für alle Werthe derselben constant, folglich wird sich $\sqrt[m]{ }^{4}$ der Einheit immer melur und mehr 
nähern, und zuletzt so ganz damit zusammenfallen, dafs die Bedingung $g>g^{\prime} \sqrt{ }^{4}$ übergeht in $g>g^{\prime}$. So z. B. ist für $\boldsymbol{m}=128$

$$
\stackrel{m}{r}^{4}=1,011 \text {. }
$$

Es geht demnach, sobald $g>g^{\prime}$ der Coefficient von $x^{2 n-2}$,

$$
\left[g^{2 m}\right]+\left[f_{m} f_{m}^{\prime}\right] \text { über in } g^{2 m} \text {. }
$$

Ganz dieselben Schlüsse lassen sich bei allen Coefficienten machen, welche mit geraden Potenzen verbunden sind, und man braucht bei ihnen immer nur die Summen, welche lanter $g$ enthalten, zu vergleichen mit den Summen, in welchen zwei $f$ mii den $g$ verbunden sind. Kommen nämlich mehr $f$ als zwei in der Summe vor, so gehören sie nothwendig zu kleineren $g$ als die sind, welche in den Summen vorkommen die nur $g$ enthalten. Im Allgemeinen werden mit vergröfsertem $m$ die Coefficienten der geraden Potenzen von $x$, ganz wie bei den reellen Wurzeln, übergehen in

$$
g^{2 m}, g^{2 m} g^{\prime 2 m}, \quad g^{2 m} g^{\prime 2 m} g^{\prime \prime 2 m} \text {, etc. }
$$

Die Coefficienten der ungeraden Potenzen von $x$ enthalten kein Glied, in welchem nicht wenigstens ein $f_{m}$ vorkäme, und da jedes solche $f_{m}$ schwankende Werthe hat, selbst Null werden kann, oder doch einen sehr kleinen Werth erhalten, so können diese Coefficienten auch bei noch so grofsem $m$ nie einer bestimmten Grenze sich nähern, den Ausnahmefall ausgenommen, wenn $m \emptyset$ ein Vielfaches von $\pi$ ist, welcher, da er mit den gleichen reellen Wurzeln zusammenfällt, später betrachtet werden soll. Bei lauter inaginären Wurzeln und ungleichen Moduln wechselt ein unbestimmtes Glied stets ab mit einem solchen, welches den Werth eines neuen $g^{2 m} \mathrm{zu}$ den vorigeii hinzufügt. Wenn folglich die Grenze, in welcher die Endform stattfindet, erreicht ist, worüber man eben so wie bei den reellen Wurzeln durch den Gang der Rechunng unterrichtet wird, so hat die Gleichung die Form

$$
\begin{gathered}
x^{2 n}+f_{0} x^{2 n-1}+g^{2 m} x^{2 n-2}+f_{0}^{\prime} x^{2 n-3}+g^{2 m} g^{j^{2 m}} x^{2 n-4}+f_{0}^{\prime \prime} x^{2 n-5} \\
+g^{2 m} g^{2 m} g^{\prime \prime^{2 m}} x^{2 n-6} \ldots \ldots=0,
\end{gathered}
$$

wo durch $f_{0}, f_{0}^{\prime}, f_{0}^{\prime \prime}$ der schwankende Werth der Coefficienten bezeichnet wird.

Man findet also ganz auf dieselbe Weise, wie bei den reellen Wurzeln ${ }_{2}$ durch successive Divisionen erst $g^{2 m}$, dann $\frac{g^{2 m} g^{2 m}}{g^{2 m}}=g^{\prime^{2 m}}$ u. s. W.o. So 
dafs jetzt bei bekannten $g$ die zu jedem Modul gehörigen $f$ noch zu bestimmen sind.

Hiezu bietet die gegebene Gleichung selbst weit mehr Bedingungsgleichungen dar, als nöthig sind, so dafs sich a priori übersehen lälst, dafs die verschiedenen $f$ jedesmal linear und ohne $\mathbf{Z}$ weideutigkeit sich bestimmen lassen müssen. Eine Gleichung vom $2 n^{\text {ten }}$ Grade, in der alle $g$ bekannt sind, enthält in ihren $2 n+1$ Gliedern $2 n$ Coefficienten, in denen die unbekannten Gröfsen $f$ an der Zahl $n$ enthalten sind, und aus welchen sie bestimmt werden können. Von diesen Coefficienten ist einer, der letzte, frei von allen $f$; von den noch übrigen $2 n-1$ sind zwei, der zweite und vorletzle, vom ersten Grade in Bezug auf die $f$; zwei, der dritte und drittletzte, vom zweiten Grade und überhaupt sind immer zwei gleich weit vom Anfange und Ende abstehende Coefficienten von gleichem Grade in Bezug auf die $f$, durch alle Grade hindurch, bis zum $(n-1)^{t_{\text {ol }}}$ inclusive; der mittelste alleinstehende aber ist vom $n^{\text {ten }}$ Grade. Man kanu deswegen zur Bestimmung der $f$ so verfahren, dafs man aus

$$
\alpha_{1}=[f] \quad \text { und } \quad a_{2 n-1}=\left[g^{2} g^{\prime 2} \ldots g^{(n-2)^{2}} f^{(n-1)}\right]
$$

zwei $f$ linear als Function der übrigen und bekannter Gröfsen bestimmt. Es mögen dieses etwa $f$ und $f^{\prime}$ sein. Substituirt man diese Werthe in

$$
\begin{aligned}
& \alpha_{2}=\left[g^{2}\right]+\left[f f^{\prime}\right], \\
& \alpha_{2 n-2}=\left[g^{2} g^{\prime 2} \ldots g^{(n-2)^{2}}\right]+\left[g^{2} g^{\prime 2} \ldots g^{(n-3)^{2}} f^{(n-2)} f^{(n-1)}\right],
\end{aligned}
$$

so hat man zwei Gleichungen vom zweiten Grade, aus deren Verbindung sich ein drittes $f$, etwa $f^{\prime \prime}$, linear als Function der übrigen finden läfst. Dieses geschieht unmittelbar durch die Division beider Gleichungen in einander, bis ein Ausdruck übrig bleibt, der nur noch die erste Potenz von $f^{\prime \prime}$ enthält. Die Substitution dieses Werthes in eine der Gleichungen, aus der er hervorging, wird eine Gleichung vom $4^{\text {ten }}$ Grade geben, aus welcher die drei $f f^{\prime} f^{\prime \prime}$ verschwunden sind, und wenn man die Werthe dieser drei Gröfsen in die Coefficienten von $x^{2 n-3}$ und $x^{3}$ substituirt, so hat man zwei neue Gleichungen vom $6^{\text {ten }}$ Grade, die in Verbindung mit der vom $4^{\text {ten }}$ Grade wieder linear zwei neue $f$ als Functionen der übrigen bestimmen lassen müssen. Allein auf diesem Wege wird man doch höchsteus noch ein viertes $f$, etwa $f^{\prime \prime \prime}$, bestimmen kömnen, oder also nur in dem Falle von 8 imaginären Wurzeln Gebrauch davon machen können. Denn die Elimination von $f^{\prime \prime \prime}$ aus einer Gleichung vom $4^{\text {ten }}$ und vom $6^{\text {ten }}$ 
Grade wird mindestens zu einer Gleichung vom $\mathbf{2 4}^{\text {sten }}$ Grade führen, die sich nicht mehr behandeln läfst. In der Praxis wird der Grad noch höher steigen. Denn wenn man sich nicht die Mühe geben will, die symmetrischen Functionen der Wurzeln zu bilden, sondern den Weg der Division, der auch der einzige wirklich anwendbare sein möchte, wählt, so wird mas bei der Verbindung einer Gleichung vom $m^{\text {ten }}$ Grade mit einer vom $n^{\text {ten }}$ in der Regel so viele überflüssige Factoren einführen müssen, dafs die Endgleichung, in welcher die zu eliminirende Gröfse nur noch anf der ersten Potenz sich befindet, in Bezug auf die übrigen darin enthaltenen Unbekannten, vom $(m+n-2)^{\text {ten }}$ Grade ist, also durch die Substitution des aus ihr erhaltenen Werthes nothwendig einen höhern Grad als den $m n^{\text {ien }}$ erreichen läfst, wenn $m$ und $n$ die Potenz 2 ühersteigen. Man wird deshalb höchstens bis zur Bestimmung von vier $f$ diesen Weg einschlagen können.

In der That scheint es aber auch in der Natur der Aufgabe zu liegen, dafs eine gewisse Weitläufigkeit nicht zu vermeiden ist. Denn wenn auch nur $n$ Gröfsen $\boldsymbol{f}$ gesucht werden, so würde doch eine Gleichung vom $n^{\text {ten }}$ Grade allein nicht dem Probleme genügen, wenn man sie auch aufstellen könnte. Man verlangt nämlich nicht blofs die Werthe der verschiedenen $f$ selbst, sondern man verlangt den Werth eines jeden bestimmten $f$, was einem bestimmten $g$ angehört. Sonach möchte es wohl die einfachste Auflösung sein, die sich erwarten läfst, wenn man eine Gleichung angiebt, die, je vachdem man den Werth eines bestimmten $g$ in sie hinein substituirt, auch jedesmal das zugehörige $f$ giebt. Diese Gleichung mufs vom $n^{\text {ten }}$ Grade sein, da in dem Falle, dafs sämtliche Modulı $g$ einander gleich wären, während die Winkel $\phi$ und folglich die $f$ verschieden sind, die $n$ Werthe von $f$ aus den Wurzeln der Gleichung sich ergeben müfsten. Kann man damit eine älnliche Gleichung niederen Grades verbinden, die bei gleicher Substitution des bestimmten $g$ jedesmal als gemeinschaftliche Wurzel mit der ersten Gleichung den verlangten Werth von $\boldsymbol{f}$ hat, so dafs man durch einfache Division den Werth von $f$ linear findet, und läfst sich diese Division nit der gröfsten Bequemlichkeit in jedem Falle ausführen, so scheint die Aufgabe so einfach gelöst zu sein, als die Natur des Gegenstandes es erlaubt.

Solche zwei Gleichungen erlangt man auf die einfachste Weise, wenn man die allgemeine Form der imaginären Wurzeln in die gegebene 
Gleichung hinein substituirt. Ein Werth

$$
x=r(\cos \varphi+\sin \varphi r-1)
$$

giebt, wenn man ihn in die Gleichung

$$
x^{2 n}+a_{1} x^{2 n-1}+\alpha_{2} x^{2 n-2} \ldots .+a_{2 n}=0
$$

setzt, durch Trennung des Imaginären vom Reellen, oder, indem man den zweiten Werth $x=r(\cos \varphi-\sin \varphi \sqrt{ }-1)$ ebenfalls einführt, und die Resultate beider Substitutionen verbindet, zwei Gleichungen:

$$
\begin{aligned}
0=r^{2 n} \cos 2 n \varphi+\alpha_{1} r^{2 n-1} \cos (2 n-1) \varphi & +\alpha_{2} r^{2 n-2} \cos (2 n-2) \emptyset \ldots \\
& +\alpha_{2 n-1} r \cos \varphi+\alpha_{2 n} \\
0=r^{2 n} \sin 2 n \varphi+\alpha_{1} r^{2 n-1} \sin (2 n-1) \varphi & +\alpha_{2} r^{2 n-2} \sin (2 n-2) \varphi \ldots \\
& +\alpha_{2 n-1} r \sin \emptyset .
\end{aligned}
$$

Multiplicirt man die erste mit $\cos n \emptyset$ und die zweite mit $\sin n \phi$ und addirt beide Producte, und multiplicirt man nachher auch die erste mit $\sin n \emptyset$ und die zweite mit $\cos n \emptyset$ und subtrahirt das erste Product vom zweiten, so erhält man die zwei Gleichungen :

$$
\begin{aligned}
0=r^{2 n} \cos n \varphi & +\alpha_{1} r^{2 n-1} \cos (n-1) \varphi+\alpha_{2} r^{2 n-2} \cos (n-2) \emptyset \ldots \\
& +\alpha_{2 n-2} r^{2} \cos (n-2) \varphi+\alpha_{2 n-1} r \cos (n-1) \emptyset+\alpha_{2 n} \cos n \emptyset \\
0=r^{2 n} \sin n \varphi & +\alpha_{1} r^{2 n-1} \sin (n-1) \emptyset+\alpha_{2} r_{2 n-2} \sin (n-2) \varphi \ldots \\
& -\alpha_{2 n-2} r^{2} \sin (n-2) \varphi-\alpha_{2 n-1} r \sin (n-1) \emptyset-\alpha_{2 n} \sin n \varphi .
\end{aligned}
$$

In diesen enthalten immer die gleich weit vom Ende und vom Anfange abstehenden Glieder einerlei Sinus und Cosinus. Vereinigt man diese und setzt man also

$$
\begin{array}{cccc}
1+\alpha_{2 n} \boldsymbol{r}^{-2 n} & =\beta & 1-\alpha_{2 n} \boldsymbol{r}^{-2 n} & =\gamma \\
\alpha_{2}+\alpha_{2 n-1} \boldsymbol{r}^{-(2 n-2)} & =\beta_{1} & \alpha_{1}-\alpha_{2 n-1} \boldsymbol{r}^{-(2 n-2)} & =\gamma_{2} \\
\alpha_{2}+\alpha_{2 n-2} \boldsymbol{r}^{-(2 n-4)} & =\beta_{2} & \alpha_{2}-\alpha_{2 n-2} \boldsymbol{r}^{-(2 n-4)} & =\gamma_{2} \\
\vdots & & \vdots \\
\alpha_{n-1}+\alpha_{n+1} \boldsymbol{r}^{-2} & =\beta_{n-1} & \alpha_{n-1}-\alpha_{n+1} \boldsymbol{r}^{-2} & =\gamma_{n-1} \\
\alpha_{n}+\alpha_{n} & =\beta_{n}, & &
\end{array}
$$

so werden die beiden Gleichungen, wenn man sie mit $\boldsymbol{r}^{2 n}$ dividirt,

$$
\begin{aligned}
& 0=\beta \cos n \varphi+\frac{\beta_{1}}{r} \cos (n-1) \varphi+\frac{\beta_{2}}{r^{2}} \cos (n-2) \varphi \ldots+\frac{\beta_{n-1}}{r^{n-1}} \cos \varphi+\frac{\beta_{n}}{2 r^{n}} \\
& 0=\gamma \sin n \varphi+\frac{\gamma_{1}}{r} \sin (n-1) \varphi+\frac{\gamma_{2}}{r^{2}} \sin (n-2) \emptyset \ldots+\frac{\gamma_{n-1}}{r^{n-1}} \sin \varphi .
\end{aligned}
$$

Es lassen sich aber durch die bekannten Reihen die Cosinus und Sinus des vielfachen Winkels als Functionen der Cosinus und Sinus des einfachen ausdrücken, und für $n$ gleich einer ganzen positiven Zahl ist 
8. Encke, über allgemeine Auflösung der numerischen Gleichungen. 209 allgemein, wenn man alle negativen Potenzen der Cosinus und Sinus des einfachen Winkels weglä[st:

$$
\begin{aligned}
\cos n \emptyset & =2^{n-1} \cos \phi^{n}-\frac{n}{1} 2^{n-3} \cos \phi^{n-2}+\frac{n(n-3)}{1.2} 2^{n-5} \cos \phi^{n-4} \\
& -\frac{n(n-4)(n-5)}{1.2 .3} 2^{n-7} \cos \phi^{n-6}+\frac{n(n-5)(n-6)(n-7)}{1.2 .3 .4} 2^{n-9} \cos \phi^{n-8}-\text { etc. }
\end{aligned}
$$

$\frac{\sin n \varphi}{\sin \varphi}=2^{n-1} \cos \varphi^{n-1}-\frac{n-2}{1} 2^{n-3} \cos \varphi^{n-3}+\frac{(n-3)(n-4)}{1.2} 2^{n-5} \cos \varphi^{n-5}$

$-\frac{(n-4)(n-5)(n-6)}{1.2 .3} 2^{n-7} \cos \phi^{n-7}+\frac{(n-5)(n-6)(n-7)(n-8)}{1.2 .3 .4} 2^{n-9} \cos \phi^{n-9}$ - etc.

Substituirt man diese Reihen in den beideu letzten Gleichungen, so erhält man:

$0=2^{n-1} \beta \cos \varphi^{n}+\frac{\beta_{1}}{r} 2^{n-2} \cos \varphi^{n-1}+\frac{\beta_{2}}{r^{4}} 2^{n-3} \cos \varphi^{n-2}$

$$
\begin{gathered}
-\frac{n}{1} 2^{n-3} \beta \cos \phi^{n-2} \\
+\frac{\beta_{3}}{r^{3}} 2^{n-4} \cos \phi^{n-3}+\frac{\beta_{4}}{r^{4}} 2^{n-5} \cos \phi^{n-4} \\
-\frac{n-1}{1} \frac{\beta_{1}}{r} 2^{n-4} \cos \phi^{n-3}-\frac{n-2}{1} \frac{\beta_{2}}{r^{2}} 2^{n-5} \cos \phi^{n-4} \\
+\frac{n . n-3}{1.2} \beta 2^{n-5} \cos \phi^{n-4}+\ldots \text { etc. }
\end{gathered}
$$

$0=2^{n-1} \gamma \cos \phi^{n-1}+\frac{\gamma_{1}}{r} 2^{n-2} \cos \phi^{n-2}+\frac{\gamma_{2}}{r^{2}} 2^{n-3} \cos \phi^{n-3}$

$$
\begin{gathered}
-\frac{n-2}{1} \gamma 2^{n-3} \cos \phi^{n-3} \\
+\frac{\gamma_{3}}{r^{3}} 2^{n-4} \cos \phi^{n-4}+\frac{\gamma_{4}}{r^{4}} 2^{n-5} \cos \varphi^{n-5} \\
-\frac{n-3}{1} \frac{\gamma_{1}}{r} 2^{n-4} \cos \phi^{n-4}-\frac{(n-4)}{1} \frac{\gamma_{2}}{r^{2}} 2^{n-5} \cos \varphi^{n-5} \\
-\frac{(n-3)(n-4)}{1.2} \gamma 2^{n-5} \cos \phi^{n-5}+\ldots \text { etc. }
\end{gathered}
$$

Man wünscht aber eigentlich nicht $\cos \emptyset$ zu erhalten, sondern die Grörse $f$ des trinomischen Factors der beiden imaginären Wurzeln, also nach der angenommenen Form $x=r(\cos \varphi \pm \sin \varphi r-1)$ die Grölse

$$
-2 r \cos \varphi=t \text {. }
$$

Multiplicirt man deshalb, um $t$ als Wurzel zu bekommen, die Glieder beider Gleichungen nacheinander mit

$$
(-2 r)^{0},(-2 r)^{1},(-2 r)^{2},(-2 r)^{3} \ldots
$$

so werden sich beide Gleichungen durch $2^{n-1}$ dividiren lassen, und die Crelle's Journal d. M. Bd. XXI. IIn. 3. 
Endform wird mit Weglassung aller negativen Potenzen von $t$ sein:

$$
\begin{aligned}
0=\beta t^{n} & -\beta_{1} t^{n-1}+\beta_{2} t^{n-2}-\beta_{3} t^{n-3} \ldots \pm \beta_{n} \\
& -r^{2}\left\{n \beta t^{n-2}-(n-1) \beta_{1} t^{n-3}+(n-2) \beta_{2} t^{n-4}-\ldots\right\} \\
& +r^{4}\left\{\frac{n(n-3)}{1.2} \beta t^{n-4}-\frac{(n-1)(n-4)}{1.2} \beta_{1} t^{n-5}+\frac{(n-2)(n-5)}{1.2} \beta_{2} t^{n-6}-\ldots\right\} \\
& -r^{6}\left\{\frac{n(n-4)(n-5)}{1.2 .3} \beta t^{n-6}-\frac{(n-1)(n-5)(n-6)}{1.2 .3} \beta_{1} t^{n-7} \ldots\right\} \\
& +r^{s}\left\{\frac{n(n-5)(n-6)(n-7)}{1.2 .3 .4} \beta t^{n-8} \ldots\right\}-\text { etc. } \\
0=\gamma t^{n-1} & -\gamma_{1} t^{n-2}+\gamma_{2} t^{n-3}-\gamma_{3} t^{n-4} \ldots \pm \gamma_{n-1} \\
& -r^{2}\left\{(n-2) \gamma t^{n-3}-(n-3) \gamma_{1} t^{n-4}+(n-4) \gamma_{2} t^{n-5} \ldots\right\} \\
& +r^{4}\left\{\frac{(n-3)(n-4)}{1.2} \gamma t^{n-5}-\frac{(n-4)(n-5)}{1.2} \gamma_{1} t^{n-6} \ldots\right\} \\
& \left.-r^{6}\left\{\frac{(n-4)(n-5)(n-6)}{1.2 .3} \gamma t^{n-7}-\frac{(n-5)(n-6)(n-7)}{1.2 .3} \gamma_{1} t^{n-8} \ldots\right\}\right\} \\
& +r^{8}\left\{\frac{(n-5)(n-6)(n-7)(n-8)}{1.2 .3 .4} \gamma t^{n-9} \ldots\right\}-\text { etc. }
\end{aligned}
$$

Wenn in die Werthe der $\beta$ und $\gamma$, und in diese beiden Gleicliungen für $\boldsymbol{r}$ ein bestimmites $g$ substituirt ist, so werden beide Gleiclungen eine gemeinschafliche Wurzel für $t$ geben müssen, welche nichts anders als der Werth des $f$ ist, was zu dem substituirten $g$ gehört. Die sämtlichen Ausdrücke sind höchst einfach. Der Grad der Gleichungen ist nicht höher als unumgänglich nöthig, und die Division läfst sich, wie sogleich gezeigt werden wird, mit der gröfsten Leichtigkeit vermittelst der Logarithmen ausführen, so dafs man ohne Mühe aus dem gemeinschaftlichen linearen Divisor beider Gleichungen den Werth von $f$ ohne Zweideutigkeit erhält. Gehören mehrere $f$ zu demselben Werthe von $g$, wenn nämlich mehrere Paare imaginärer Wurzeln gleiche Moduln haben, so wird der gcmeinschaftliche Divisor ein quadratischer, oder cubischer. Die Wurzeln müssen in diesem Falle stets reell sein und die verschiedenen Werthe für die eiızelnen $f$ geben.

Für die einfacheren gewöhnlichen Fälle sind die Formeln folgende:

Vier imaginäre Wurzeln.

$$
\begin{array}{rlrl}
1+\alpha_{4} r^{-4} & =\beta & 1-\alpha_{4} r^{-4} & =\gamma \\
\alpha_{1}+\alpha_{3} r^{-2} & =\beta_{1} & \alpha_{1}-\alpha_{3} r^{-2}=\gamma_{1} \\
2 \alpha_{2} & =\beta_{2} & \\
0 & =\beta t^{2}-\beta_{1} t+\beta_{2}-2 \beta r^{2} \\
0 & =\gamma t-\gamma_{1} .
\end{array}
$$


8. Encke, über allgemeine Auflösung der numerischen Gleichungen. 211

Hier reicht die letzte Gleichung schon allein aus. Die erste kann als Prüfung der Rechnung gebraucht werden.

$$
\begin{aligned}
& \text { Sechs imayinäre Wurseln. } \\
& 1+a_{6} r^{-6}=\beta \quad 1-a_{6} r^{-6}=\gamma \\
& a_{1}+a_{5} r^{-4}=\beta_{1} \quad \alpha_{1}-a_{5} r^{-4}=\gamma_{1} \\
& \alpha_{2}+a_{4} r^{-2}=\beta_{2} \quad a_{2}-\alpha_{4} r^{-2}=\gamma_{2} \\
& 2 \alpha_{3}=\beta_{3} \\
& 0=\beta t^{3}-\beta_{1} t^{2}+\left(\beta_{2}-3 \beta r^{2}\right) t-\left(\beta_{3}-2 \beta_{1} r^{2}\right) \\
& 0=\gamma t^{2}-\gamma_{1} t+\gamma_{2}-\gamma r^{2} \text {. } \\
& \text { Acht imaginüre Wurzeln. } \\
& 1+a_{8} r^{-8}=\beta \quad 1-a_{8} r^{-8}=\gamma \\
& \alpha_{1}+\alpha_{7} r^{-6}=\beta_{1} \quad \alpha_{1}-\alpha_{7} r^{-6}=\gamma_{2} \\
& a_{2}+a_{i j} r^{-4}=\beta_{2} \quad a_{2}-a_{6} r^{-4}=\gamma_{2} \\
& a_{3}+a_{5} \boldsymbol{r}^{-2}=\beta_{3} \quad \alpha_{3}-a_{5} \boldsymbol{r}^{-2}=\gamma_{3} \\
& 2 \alpha_{4}=\beta_{4} \\
& 0=\beta t^{4}-\beta_{1} t^{3}+\left(\beta_{2}-4 \beta r^{2}\right) t^{2}-\left(\beta_{3}-3 \beta_{1} r^{2}\right) t+\beta_{4}-2 \beta_{2} r^{2}+2 \beta r^{4} \\
& 0=\gamma t^{3}-\gamma_{1} t^{2}-\left(\gamma_{2}-2 \gamma r^{2}\right) t-\left(\gamma_{3}-\gamma_{1} r^{2}\right) \text {. }
\end{aligned}
$$

Es würde keine Mühe machen, die Division in Zeichen wirklich auszuführen und den Ausdruck von $t$ als Function von $r^{2}$ und den $\beta$ und $\gamma$ hinzusetzen. Allein es ist weit bequemer, die Division numerisch zu macheu. Denn vermittelst der Gaufsischen Tafeln, welche aus den Logarithmen zweier Zahlen sogleich durch einmaliges Eingehen den Logarithmen der Summe und Differenz ganz strenge finden lassen; und die besonders für Logarithmen von 5 Decimalen höchst bequem sind (für 7 Decimalen ist mir der Gebrauch dieser Tafeln nicht so bequem vorgekommen, doch kaun es Mangel an Uebung sein), dividirt man solche Gleichungen mit einer Leichtigkeit in einander, die nichts $\mathrm{zu}$ wünschen übrig läfst.

Bei den Gaufsischen Tafeln wird der Logarithme von $a \pm b$ gefunden dadurch, dafs man zu dem Logarithmen der gröfsten Zahl eine aus den Tafeln geuommene hinzulegt oder abzieht. Die Form ist also allgemein

$$
\lg (a \pm b)=\lg a \pm B
$$

wo $B$ mit $\lg a-\lg b$ gefunden wird. Man bestimme nun die Logaritlımen sämtlicher Coefficienten beider Gleichungen, und bringe sie durch $\mathbf{A b}$ ziehen von $\lg \beta$ in der ersten, und $\lg \gamma$ in $\operatorname{der}$ zweiten Gleichung auf 
8. Encke, über allgemeine Auflösung der numerischen Gleichungen.

die Form

$$
\begin{aligned}
& 0=t^{n}-\delta t^{n-1}+\delta^{\prime} t^{n-2}-\delta^{\prime \prime} t^{n-3} \ldots \\
& 0=t^{n-1}-\varepsilon t^{n-2}+\varepsilon^{\prime} t^{n-3}-\varepsilon^{\prime \prime} t^{n-4} \ldots .
\end{aligned}
$$

Hier bedeuten die $\delta$ und $\varepsilon$ die Logarithmen der Coefficienten, denen die Zeichen ebenso wie den Zahlen vorgesetzt werden. Geht man nun mit $\delta-\varepsilon$ oder $\varepsilon-\delta$ in die Gaufsischen Tafeln ein, und ebenso nit $\delta^{\prime}-\varepsilon^{\prime}$ oder $\varepsilon^{\prime}-\delta^{\prime}$ etc., so erhält man die verschiedenen $\boldsymbol{B}$, die gehörig unter $\varepsilon \varepsilon^{\prime} \varepsilon^{\prime \prime}$ gesetzt werden, und nach den Zeichen mit dem gröfseren Logarithmen verbunden geben

$$
0=\zeta^{n-1}+\zeta^{\prime} t^{n-2}+\zeta^{\prime \prime} t^{n-3} \ldots .
$$

Diese Gleichung bringt man wieder durch Abziehen von $\zeta$ auf die Form

$$
0=t^{n-1}+\theta t^{n-2}+\theta^{\prime} t^{n-3} \ldots \text { etc. }
$$

und verbindet sie auf dieselbe Weise mit der Gleichung vom $(n-1)^{\text {ien }}$ Grade. Auf dieselbe Weise fährt man fort, bis man zu dem linearen gemeinschaftlichen Factor kommt, der gleich Null gesetzt, den Werth von $f$ giebt. Das Schema ist also folgendes:

$$
\begin{aligned}
& 0=\beta t^{n}-\beta_{1} t^{n-1}+\left(\beta_{2}-n \beta r^{2}\right) t^{n-2} \ldots \ldots \ldots \ldots \text { etc. } \\
& 0=\gamma t^{n-1}-\gamma_{1} t^{n-2}+\left(\gamma_{2}-(n-2) \gamma r^{2}\right) t^{n-3} \ldots \ldots \text { etc. }
\end{aligned}
$$

Hieraus werden zuerst die Logarithmen statt der Zahlen gesetzt, die Zeichen der Zahlen aber beibehalten, und dann wird nach und nach gebildet

$$
\begin{aligned}
& 0=t^{n}+\delta t^{n-1}+\delta^{\prime} t^{n-2}+\delta^{\prime \prime} t^{n-3} \ldots \\
& 0=t^{n-1}+\varepsilon t^{n-2}+\varepsilon^{\prime} t^{n-3}+\varepsilon^{\prime \prime} t^{n-4} \ldots \\
& \boldsymbol{B} \boldsymbol{B}^{\prime} \quad \boldsymbol{B}^{\prime \prime} \ldots \\
& \theta t^{n-1}+\theta^{\prime} t^{n-2}+\theta^{\prime \prime} t^{n-3} \ldots \\
& 0=t^{n-1}+\zeta t^{n-2}+\zeta^{\prime} t^{n-3}+\zeta^{\prime \prime} t^{n-4} \ldots . \\
& \begin{array}{lll}
B_{1} & B_{1}^{\prime} & B_{1}^{\prime \prime} \ldots
\end{array} \\
& \eta t^{n-2}+\eta^{\prime} t^{n-3}+\eta^{\prime \prime} t^{n-4} \ldots \\
& 0=t^{n-2}+t^{n-3}+\iota^{\prime} t^{n-4}+\iota^{\prime \prime} t^{n-5} \ldots . \\
& \boldsymbol{B}_{2} \quad \boldsymbol{B}_{2}^{\prime} \quad \boldsymbol{B}_{2}^{\prime \prime}, \ldots \\
& u t^{n-2}+u^{\prime} t^{n-3}+u^{\prime \prime} t^{n-4} \ldots \\
& 0=t^{n-2}+\lambda t^{n-3}+\lambda^{\prime} t^{n-4}+\lambda^{\prime \prime} t^{n-5} \ldots .
\end{aligned}
$$

Die einzige Tafel, die man gebraucht bei dieser Division, ist die Gaufsische für Differenz und Summe der Logarithmen, und die HauptAufnerksamkeit wird auf die Zeichen gerichtet werden müssen, um gehörig 
zu addireu oder zu subtrahiren, und dem Resultate sein ihm zukommendes Zeichen zu geben.

Die beiden Gleichungen zwischen $t$ und $r$ haben aber noch eine weitere Bedeutung, die für die Auflösung der Gleichungen im allgemeinen von Wichtigkeit ist. Denn wenn gleich sie aus der Form der imaginären Wurzeln abgeleitet sind, so gelten sie doch für alle trinomische Factoren, auch für die, deren Wurzeln reell sind. Wenn für irgend welchen trinomischen Factor

$$
x^{2}+t x+v
$$

der Werth von $v$ bekannt geworden ist, und man substituirt ihn an die Stelle der $\boldsymbol{r}^{2}$ in $\beta, \gamma$ und in die beiden Gleichungen, so giebt die Division beider in einander den Werth von $t$, der zu $v$ gehört. Man kann nämlich die beiden linearen Factoren von $x^{2}+t x+v$ jedesmal darstellen unter der Form

$$
(x+y \sqrt{ } v)\left(x+\frac{1}{y} \sqrt{ } v\right)
$$

Denn für ein positives $v$, oder $\sqrt{v}=$ einer reellen Grörse $g$, wird

$$
t=g\left(y+\frac{1}{y}\right)
$$

und da $t$ in diesem Falle die Summe beider Wurzeln ist, so werden die Wurzeln selbst $g y$ und $g \frac{1}{y}$, oder wenn man sie nit $a$ und $b$ bezeichnet, so wird

$$
y=\sqrt{\frac{a}{b}}, \quad \frac{1}{y}=\sqrt{\frac{b}{a}}, \quad g=\sqrt{a} b .
$$

Diese Form gilt für reelle Wurzelı, welche, wegen $v$ positiv, gleiches Zeichen haben müssen, so wie für imaginäre, bei welchen letzteren:

$$
y=\sqrt{\frac{\alpha+\beta \sqrt{ }-1}{\alpha-\beta \sqrt{-1}}}=\frac{\alpha+\beta \sqrt{ }-1}{\sqrt{\left(\alpha^{2}+\beta^{2}\right)}}, \quad \frac{1}{y}=\frac{\alpha-\beta \sqrt{ }-1}{\sqrt{\left(\alpha^{2}+\beta^{2}\right)}}, \quad g=\sqrt{ }\left(\alpha^{2}+\beta^{2}\right) .
$$

Wenn aber $v$ negativ, der Fall, wo die Wurzeln stets reell sein müssen, aber verschiedenes Zeichen haben, so wird

$$
y=-r-1 \sqrt{\frac{a}{b}}, \quad \frac{1}{y}=r-1 \sqrt{\frac{b}{a}}, \quad g=r-1 \sqrt{a b},
$$

und folglich

$$
t=a-b,
$$

wie es hier sein mufs. Substituirt man nun in die Gleichung

die beiden Werthe von $x$

$$
x^{2 n}+\alpha_{1} x^{2 n-1}+\alpha_{2} x^{2 n-2} \ldots \ldots+\alpha^{2 n}=0
$$

$$
x=-g y \quad \text { und } \quad x=-\frac{5}{y},
$$


214 8. Encke, über allgemeine Auflösung der numerischen Gleichungen.

so erhält man zwei Gleichungen

$$
\begin{aligned}
g^{2 n} y^{2 n}-\alpha_{1} g^{2 n-1} y^{2 n-1} & +\alpha_{2} g^{2 n-2} y^{2 n-2}-\ldots \\
& +\alpha_{2 n-2} g^{2} y^{2}-\alpha_{2 n-1} g y+a_{2 n}=0 \\
g^{2 n} y^{-2 n}-a_{1} g^{2 n-1} y^{-(2 n-1)} & +a_{2} g^{2 n-2} y^{-(2 n-2)}-\ldots . \\
& +a_{2 n-2} g^{2} y^{-2}-\alpha_{2 n-1} g y^{-1}+a_{2 n}=0 .
\end{aligned}
$$

Man multiplicire die erste mit $g^{-2 n} y^{-n}$, die zweile mit $g^{-2 n} y^{n}$, so werden sie

$$
\begin{aligned}
& y^{n}-\alpha_{1} g^{-1} y^{n-1}+\alpha_{2} g^{-2} y^{n-2}-\ldots \\
& \quad-\alpha_{2 n-2} g^{-(2 n-2)} y^{-(n-2)}-\alpha_{2 n-1} g^{-(2 n-1} y^{-(n-1)}+\alpha_{2 n} g^{-2 n} y^{-n}=0 \\
& y^{-n}-\alpha_{1} g^{-1} y^{-(n-1)}+\alpha_{2} g^{-2} y^{-(n-2)}-\ldots . \\
& \quad+\alpha_{2 n-2} g^{-(2 n-2)} y^{n-2}-\alpha_{2 n-1} g^{-(2 n-1)} y^{n-1}+\alpha_{2 n} g^{-2 n} y^{n}=0 .
\end{aligned}
$$

Legt man diese beiden letzten zusammen, und subtralirt sie von einander, so erhält man die zwei folgenden Gleichungen :

und

$$
\begin{gathered}
\left(y^{n}+y^{-n}\right)-a_{1} g^{-1}\left(y^{n-1}+y^{-(n-1)}\right)+a_{2} g^{-2}\left(y^{n-2}+y^{-(n-2)}\right)-\ldots \\
+a_{2 n-2} g^{-(2 n-2)}\left(y^{n-2}+y^{-(n-2)}\right)-a_{2 n-1} g^{-(2 n-1)}\left(y^{n-1}+y^{-(n-1)}\right) \\
+a_{2 n} g^{-2 n}\left(y^{n}+y^{-n}\right)=0
\end{gathered}
$$

$$
\begin{gathered}
\left(y^{n}-y^{-n}\right)-a_{1} g^{-1}\left(y^{n-1}-y^{-(n-1)}\right)+\alpha_{2} g^{-2}\left(y^{n-2}-y^{-(n-2)}\right)-\ldots \\
-\alpha_{2 n-2} g^{-(2 n-2)}\left(y^{n-2}-y^{-(n-2)}\right)+\alpha_{2 n-1} g^{-(2 n-1)}\left(y^{n-1}-y^{-(n-1)}\right) \\
-\alpha_{2 n} g^{-2 n}\left(y^{n}-y^{-n}\right)=0 .
\end{gathered}
$$

Um hier die Glieder, welche gleich weit ab vom Anfang und Ende stehen, und die einerlei Potenz von $y$ angehören, zu vereinigen, setze man wie oben:

$$
\begin{array}{cccc}
1+\alpha_{2 n} g^{-2 n} & =\beta & 1-\alpha_{2 n} g^{-2 n}=\gamma \\
\alpha_{1}+\alpha_{2 n-1} g^{-(2 n-2)} & =\beta_{1} & \alpha_{1}-\alpha_{2 n-1} g^{-(2 n-2)}=\gamma_{1} \\
\alpha_{2}+\alpha_{2 n-2} g^{-(2 n-4)} & =\beta_{2} & \alpha_{2}-\alpha_{2 n-2} g^{-(2 n-4)}=\gamma_{2} \\
\vdots & & \vdots & \\
\alpha_{n-1}+\alpha_{n+1} g^{-2} & =\beta_{n-1} & \alpha_{n-1}-\alpha_{n+1} g^{-2} & =\gamma_{n-1} \\
2 \alpha_{n} & =\beta_{n}, & &
\end{array}
$$

so erhält man die Form

$$
\begin{aligned}
\beta\left(y^{n}+y^{-n}\right)-\frac{\beta_{1}}{g}\left(y^{n-1}+y^{-(n-1)}\right) & +\frac{\beta_{2}}{g^{2}}\left(y^{n-2}+y^{-(n-2)}\right)-\ldots \\
& \pm \frac{\beta_{n-1}}{g^{n-1}}\left(y+y^{-1}\right) \mp \beta_{n}=0 \\
\gamma\left(y^{n}-y^{-n}\right)-\frac{\gamma_{1}}{g}\left(y^{n-1}-y^{-(n-1)}\right) & +\frac{\gamma_{2}}{g^{2}}\left(y^{n-2}-y^{-(n-2)}\right)-\ldots \\
& \pm \frac{\gamma_{n-1}}{g^{n-1}}\left(y-y^{-1}\right)=0 .
\end{aligned}
$$


8. Enke, ̈̈ber allgemeine Auflösung der numerischen Gleichungen.

Es haben nun aber die hier vorkommenden Functionen die Eigenschaft, wovon man sich durch unmittelbare Rechnung überzeugen kann, dafs:

$$
\begin{aligned}
& y^{n}+y^{-n}=\left(y+y^{-1}\right)\left(y^{n-1}+y^{-(n-1)}\right)-\left(y^{n-2}+y^{-(n-2)}\right) \\
& y^{n}-y^{-n}=\left(y+y^{-1}\right)\left(y^{n-1}-y^{-(n-1)}\right)-\left(y^{n-2}-y^{-(n-2)}\right)
\end{aligned}
$$

für welche letztere Gleichung man auch schreiben kann

$$
\frac{y^{n}-y^{-n}}{y-y^{-1}}=\left(y+y^{-1}\right)\left(\frac{y^{n-1}-y^{-(n-1)}}{y-y^{-1}}\right)-\left(\frac{y^{n-2}-y^{-(n-2)}}{y-y^{-1}}\right)
$$

fängt man also von den einfachsten Functionen dieser Art an, so ist

$$
\begin{aligned}
& y^{0}+y^{-0}=2 \\
& y+y^{-1}=\frac{t}{g} \text { nach dem Obigen } \\
& y^{2}+y^{-2}=\frac{t^{2}}{g^{2}}-2 \\
& y^{3}+y^{-3}=\frac{t^{3}}{g^{3}}-3 \frac{t}{y} \\
& y^{4}+y^{-4}=\frac{t^{4}}{g^{4}}-4 \frac{t^{2}}{y^{2}}+2
\end{aligned}
$$

und allgemein, was sich ebenfalls durch Prüfung bei dem Uebergange von $n$ auf $n+1$ zeigen läfst, mit Weglassung aller negativen Potenzen von $t:$

$$
y^{n}+y^{-x}=\frac{t^{n}}{g^{n}}-n \frac{t^{n-2}}{g^{n-2}}+\frac{n(n-3)}{1.2} \frac{t^{n-4}}{g^{n-4}}-\frac{n(n-4)(n-5)}{1.2 .3} \frac{t^{n-6}}{g^{n-1}} \ldots
$$

eine Reihe, die ganz der obigen Cosinus-Reihe entspricht, da ihre Ableitung auch völlig identisch mit der von der Reihe für $\cos n \emptyset$ ist.

Eben so ist

$$
\begin{aligned}
& \frac{y^{0}-y^{-0}}{y-y^{-1}}=0 \\
& y-y^{-1} \\
& \frac{y-y^{-1}}{y-1} \\
& \frac{y^{2}-y^{-2}}{y-y^{-1}}=\frac{t}{g} \\
& \frac{y^{3}-y^{-3}}{y-y^{-1}}=\frac{t^{2}}{g^{2}}-1 \\
& \frac{y^{4}-y^{-4}}{y-y^{-1}}=\frac{t^{3}}{g^{3}}-2 \frac{t}{g} \\
& \frac{y^{5}-y^{-5}}{y-y^{-1}}=\frac{t^{4}}{g^{4}}-3 \frac{t^{2}}{g^{2}}+1
\end{aligned}
$$


und allgemein mit Weglassung aller negativen Potenzen von $\boldsymbol{t}$ :

$\frac{y^{n}-y^{-n}}{y-y^{-1}}=\frac{t^{n-1}}{g^{n-1}}-(n-2) \frac{t^{n-3}}{g^{n-3}}+\frac{(n-3)(n-4)}{1.2} \frac{t^{n-5}}{g^{n-5}}-\frac{(n-4)(n-5)(n-6)}{1.2 .3} \frac{t^{n-7}}{g^{n-7}}$.

Eine Reihe, die wiederum mit der obigen Reihe für $\frac{\sin n \varphi}{\sin \varphi}$ der Form und Ableitung nach identisch ist.

Substituirt man nun diese Werthe in die obigen Gleichungen, und multiplicirt sie nachher mit $g^{n}$, so erhält man

$$
\begin{aligned}
\beta t^{n} & -\beta_{1} t^{n-1}+\beta_{2} t^{n-2}-\beta_{3} t^{n-3} \ldots \pm \beta_{n} \\
& -g^{2}\left\{\beta n t^{n-2}-\beta_{1}(n-1) t^{n-3}+\beta_{2}(n-2) t^{n-4} \ldots\right\} \\
& +g^{4}\left\{\beta \frac{n \cdot(n-3)}{1.2} t^{n-4}-\beta_{1} \frac{(n-1)(n-4)}{1.2} t^{n-5}+\ldots\right\} \text { etc. }=0, \\
\gamma t^{n-1} & -\gamma_{1} t^{n-2}+\gamma_{2} t^{n-3}-\gamma_{3} t^{n-4} \ldots \pm \pm \gamma_{n-1} \\
& -g^{2}\left\{\gamma(n-2) t^{n-3}-\gamma_{1}(n-3) t^{n-4}+\gamma_{2}(n-4) t^{n-3} \ldots\right\} \\
& +g^{4}\left\{\gamma \frac{(n-3)(n-4)}{1.2} t^{n-5}-\gamma_{1} \frac{(n-4)(n-5)}{1.2} t^{n-6} \ldots\right\} \text { etc. }=0,
\end{aligned}
$$

das litifst ganz die obigen Gleichungen. Es ist klar, dafs hier $g^{2}$ nur statt $v$ eingeführt ist, um $\sqrt{v}=g$ bequemer zu schreiben. Auch kommen in den sämtlichen Formeln nur Potenzen von $g^{2}$ vor. Hieruach lärst sich ganz allgemein folgender Satz aussprechen:

Wenn in einem trinomischen Factor einer Gleichung

$$
x^{2 n}+a_{1} x^{2 n-1}+a_{2} x^{2 n-2} \ldots+\alpha_{2 n}=0,
$$

der von der Form ist $x^{2}+t x+v$, die Grölse $v$ auf irgend welche Art bekannt geworden ist, so findet man das zugehörige $t$, wenn man setzt:

$$
\begin{array}{rlrl}
1+\frac{\alpha_{2 n}}{v^{n}} & =\beta & 1 & -\frac{\alpha_{2 n}}{v^{n}}=\gamma \\
\alpha_{1}+\frac{\alpha_{2 n-1}}{v^{n}=1} & =\beta_{1} & \alpha_{1}-\frac{\alpha_{2 n-1}}{v^{n-1}} & =\gamma_{1} \\
\alpha_{2}+\frac{\alpha_{2 n-2}}{v^{n-2}} & =\beta_{2} & \alpha_{2}-\frac{\alpha_{2 n-2}}{v^{n-2}}=\gamma_{2} \\
\vdots & & & \\
a_{n-1}+\frac{\alpha_{n+1}}{v} & =\beta_{n-1} & \alpha_{n-1}-\frac{\alpha_{n+1}}{v} & =\gamma_{n-1} \\
2 \alpha_{n} & =\beta_{n} & &
\end{array}
$$

und dann die gemeinschaftliche Wurzel der folgenden beiden Gleichungen, in welchen alle negativen Potenzen von $\boldsymbol{t}$ weggelassen werden müssen, auf bekannte Weise bestimmt: 


$$
\begin{aligned}
\beta t^{n} & -\beta_{1} t^{n-1}+\beta_{2} t^{n-2}-\beta_{3} t^{n-3} \ldots \ldots \pm \beta_{n} \\
& -v\left\{\beta n t^{n-2}-\beta_{1}(n-1) t^{n-3}+\beta_{2}(n-2) t^{n-4}-\ldots\right\} \\
& +v^{2}\left\{\beta \frac{n(n-3)}{1.2} t^{n-4}-\beta_{1} \frac{(n-1)(n-4)}{1.2} t^{n-5}-\ldots\right\} \\
& -v^{3}\left\{\beta \frac{n(n-4)(n-5)}{1.2 .3} t^{n-6}-\ldots\right\} \text { etc. }=0, \\
\gamma t^{n-1} & -\gamma_{1} t^{n-2}+\gamma_{2} t^{n-3}-\gamma_{3} t^{n-4} \ldots \pm \gamma_{n} \\
& -v\left\{\gamma(n-2) t^{n-3}-\gamma_{1}(n-3) t^{n-4}+\gamma_{2}(n-4) t^{n-5} \ldots\right\} \\
& +v^{2}\left\{\gamma \frac{(n-3)(n-4)}{1.2} t^{n-5}-\gamma_{1} \frac{(n-4)(n-5)}{1.2} t^{n-6}+\ldots\right\} \\
& -v^{3}\left\{\gamma \frac{(n-4)(n-5)(n-6)}{1.2 .3} t^{n-7}-\ldots\right\} \text { etc. }=0 .
\end{aligned}
$$

Aus diesem Satze folgt, um solches beiläufig zu erwähnen, eine Auflösung der Gleichungen des vierten Grades, deren Ableitung von den andern Auflösungen etwas verschieden ist. Wenn die Gleichung des vierten Grades heifst:

$$
x^{4}+\alpha_{1} x^{3}+a_{2} x^{2}+\alpha_{3} x+\alpha_{4}=0,
$$

so werden die Hülfsgröfsen $\beta$ und $\gamma$ gefunden durch:

$$
\begin{array}{rlrl}
1+\frac{\alpha_{4}}{v^{2}} & =\beta & 1-\frac{\alpha_{4}}{v^{2}}=\gamma \\
\alpha_{1}+\frac{\alpha_{3}}{v} & =\beta_{1} & \alpha_{1}-\frac{\alpha_{3}}{v}=\gamma_{1} \\
2 a_{4} & =\beta_{2} & &
\end{array}
$$

und die Bedingungsgleichungen zwischen jedem $v$ und dem zugehörigen $t$ sind:

$$
\begin{gathered}
\beta t^{2}-\beta_{1} t+\beta_{2}-2 \beta v=0 \\
y t-\gamma_{1}=0 .
\end{gathered}
$$

Die letztere lineare Gleichung giebt, wenn man die Werthe von $\gamma$ und $\gamma_{t}$ substituirt,

$$
t=\frac{\gamma_{1}}{\gamma}=\frac{\alpha_{1} v^{2}-\alpha_{3} v}{v^{2}-\alpha_{4}} .
$$

Es ist folglich die Gleichung entstanden aus dem Producte der beiden Factoren :

$$
\left(x^{2}+\frac{\alpha_{1} v^{2}-\alpha_{3} v}{v^{2}-\alpha_{4}} x+v\right)\left(x^{2}+\frac{\alpha_{1} v^{\prime 2}-\alpha_{3} v^{\prime}}{v^{\prime 2}-\alpha_{4}} x+v^{\prime}\right)=0 .
$$

Da hieraus folgt $v v^{\prime}=\alpha_{4}$, so lassen sich, wenn man diesen Werth substituirt, die Factoren auch schreiben:

$$
\left(x^{2}+\frac{\alpha_{1} v-\alpha_{3}}{v-v^{\prime}} x+v\right)\left(x^{2}+\frac{\alpha_{1} v^{\prime}-\alpha_{3}}{v^{\prime}-v} x+v^{\prime}\right)=0,
$$

Crelle's Jubrnal d. M. Bd. XXII. Hft. 3. 
deren wirkliche Multiplication giebt:

$$
x^{4}+\alpha_{1} x^{3}+\left\{v+v^{\prime}+\left(\frac{\alpha_{1} v-\alpha_{3}}{v-v^{\prime}}\right)\left(\frac{\alpha_{1} v^{\prime}-\alpha_{3}}{v^{\prime}-v}\right)\right\} x^{2}+\alpha_{3} x+v v^{\prime}=0 .
$$

Man hat also zur Bestimmung von $v$ und $v^{\prime}$ die beiden Gleichungen:

$$
\begin{gathered}
v v^{\prime}=\alpha_{4} \\
v+v^{\prime}+\left(\frac{\alpha_{1} v-\alpha_{3}}{v-v^{\prime}}\right)\left(\frac{\alpha_{1} v^{\prime}-\alpha_{3}}{v^{\prime}-v}\right)=\alpha_{2} .
\end{gathered}
$$

Führt man die Multiplication wirklich aus in der letzten Gleichung, so läfst sich Alles durch $v+v^{\prime}$ und $v v^{\prime}$ ausdrücken, denn es wird

$$
v+v^{\prime}+\frac{\alpha_{1}^{2} v v^{\prime}-\alpha_{1} \alpha_{3}\left(v+v^{\prime}\right)+\alpha_{3}^{2}}{4 v v^{\prime}-\left(v+v^{\prime}\right)^{2}}=\alpha_{2} \text {. }
$$

Setzt man also für $v v^{\prime}$ seinen Werth $\alpha_{*}$ und bezeichnet

so hat man die cubische Gleichung

$$
v+v^{\prime}=y,
$$

$$
y^{3}-\alpha_{2} y^{2}+\left(\alpha_{1} \alpha_{3}-4 \alpha_{4}\right) y-\left(\alpha_{3}^{2}-4 \alpha_{2} \alpha_{4}+\alpha_{1}^{2} \alpha_{4}\right)=0,
$$

aus deren Auflösung man $y$ findet. Ist dieses gefunden, so wird:

$$
\begin{aligned}
& v=\frac{1}{2}\left\{y+\checkmark\left(y^{2}-4 a_{4}\right)\right. \\
& v^{\prime}=\frac{1}{2}\left\{y-\sqrt{ }\left(y^{2}-4 a_{4}\right)\right.
\end{aligned}
$$

und dann $t$ und $t^{\prime}$ :

$$
\begin{aligned}
& t=\frac{1}{2}\left\{\alpha_{1}+\frac{\alpha_{1} y-2 \alpha_{3}}{\sqrt{ }\left(y^{2}-4 \alpha_{4}\right)}\right\} \\
& t^{\prime}=\frac{1}{2}\left\{\alpha_{1}-\frac{\alpha_{1} y-2 \alpha_{3}}{\sqrt{ }\left(y^{2}-4 \alpha_{4}\right)}\right\} .
\end{aligned}
$$

Die cubische Gleichung giebt für $y$ mindestens einen reellen Werth. Hat sie nur eine reelle $W$ urzel, so wird für diese jedesmal $y^{2}-4 \alpha_{\text {, }}$ eine positive Gröfse sein müssen, weil $v$ und $v^{\prime}$ jedesmal reell gewählt werden können. Dieses wird der Fall sein, wenn die ursprüngliche Gleichung 2 reelle und 2 imaginäre Wurzeln hat. Man nimmt den reellen Werth von $y$ und hat alles übrige ohne imaginäre Grölsen bestimmt. Hat die cubische Gleichung drei reelle Wurzeln, so ist entweder für alle drei $y^{2}-4 a_{4}$ eine positive Gröfse, der Fall, wenn alle Wurzeln der ursprünglichen Gleichung reell sind, wo es Sache der freien Wahl bleibt, welche Wurzel man nehmen will. Oder es ist nur eine der drei reellen Wurzeln, so grofs, dafs $y^{2}-4 a_{4}$ positiv wird, der Fall, wo alle Wurzeln der ursprünglichen Gleichungen imaginär sind. Eine solche mufs es jedesmal geben, weil $v$ und $v^{\prime}$ immer auf eine Weise reell gemacht werden können, und wenn man sie wählt, so ist die Rechnung wieder frei von imaginären Gröfsen. 
Sobald $v$ und $t$ gefunden sind, so werden die einfachen Factoren des trinomischen Factors am leichtesten auf folgende Art bestimmt, wobei drei Fälle zu unterscheiden sind:

$$
\begin{aligned}
& \text { 1) } v \text { positiv } t<2 \sqrt{v} \\
& \frac{t}{2 \sqrt{ } v}=\cos \varphi ; \quad x^{2}+t x+v=(x+\{\cos \varphi+\sin \varphi \vee-1\} \vee v) \\
& (x+\{\cos \varphi-\sin \varphi \sqrt{ }-1\} \vee v), \\
& \text { 2) } v \text { positiv } t>2 \sqrt{v} \\
& \frac{2 \sqrt{ } y}{t}=\sin \varphi ; \quad x^{2}+t x+v=\left(x+\operatorname{cotg} \frac{1}{2} \varphi \sqrt{ } v\right)\left(x+\operatorname{tg} \frac{1}{2} \varphi \sqrt{ } v\right), \\
& \text { 3) } v \text { negativ } \\
& \frac{2 \sqrt{v}}{t}=\operatorname{tg} \varphi ; \quad x^{2}+t x-v=\left(x+\operatorname{cotg} \frac{1}{2} \varphi \sqrt{ } v\right)\left(x-\operatorname{tg} \frac{1}{2} \varphi \vee v\right) .
\end{aligned}
$$

Es wird hier bei $\sqrt{ } v$ überall abgesehen von dem Zeichen, was $v$ vor sich hat, und nur seine absolute Gröfse in Betracht gezogen.

Bei der Anwendung dieses allgemeinen Salzes macht man den Grad der Gleichung immer zu einer geraden Zahl; wenn es nöthig sein sollte, durch Hinzufügung eines Factors $(x+0)$ oder durch Multiplication der Gleichung mit $x$, wobei $a_{2 n}$ dann $=$ Null wird.

Aufserdem kann bei der Benutzung der oben entwickelten Art, die $v$ durch lauter gerade Potenzen der Wurzeln zu bestimmen, der $\mathbf{Z}$ weifel entstehen, ob in den trinomischen Factoren $v$ positiv oder negativ zu nehmen ist. Will man diesem $\mathbf{Z}$ weifel ganz ausweichen, so bestimme man nicht die trinomischen Factoren der gegebenen Gleichung selbst, sondern die trinomischen Factoren der Gleichung, deren Wurzeln die Quadrate der ursprünglichen Wurzeln sind, oder der ersten unter den abgeleiteten. Diese Gleichung wird nämlich zu Wurzeln haben 1) die Quadrate der reellen Wurzeln der gegebenen Gleichung, die als Quadrate ihrer Natur nach positiv sind, und stets ein positives $v$ geben müssen; 2) die Quadrate der vollständigeu imaginären Wurzeln der gegebenen Gleichung, die den trinomischen Factor $x^{2}+2 g^{2} \cos 2 \phi x+g^{4}$ geben, und also ebenfalls ein positives $v$; 3 ) die Quadrate der unvollstäudigen imaginären Wurzelu unter den gegebenen, $x+g \vee-1, x-g \checkmark-1$, wenn solche vorhanden sind, in denen $\phi=90^{\prime \prime}$ oder überhaupt von der Form $\left(n+\frac{1}{2}\right) \pi$, diese werden den trinomischen Factor $x^{2}-2 g^{2} x+g^{4}$, also ebenfalls ein positives $v$ geben; auch kann nie der Fall eintreten, dafs die beiden einzelnen Factoreu $\left(x-g^{2}\right)\left(x-g^{2}\right)$ getrennt würden, und sich so 
mit anderen zu einem negativen $v$ verbänden, da die $v$ stets ihrer Gröfse nach geordnet erscheinen, und folglich zwei gleich grofse lineare Factoren sich nothwendig zu einem trinomischen Factor vereinigen müssen. Wenn man auf diese Weise die trinomischen Factoren der ersten abgeleiteten Gleichung, welche etwa durch

$$
x^{2}+t_{2} x+v_{2}
$$

bezeichnet werden mögen, gefunden hat, wo $v_{2}$ nothwendig stets positiv sein mufs, so hat man für die Factoren der gegebenen GIeichung

$$
v= \pm \sqrt{ } v_{2}, \quad t=\sqrt{ }\left(t_{2} \pm 2 \sqrt{ } v_{2}\right),
$$

wo die Vorzeichen zusammengehören, und wobei man auch noch den Fall, dafs $t$ positiv oder negativ sein kann, in Betracht ziehen mufs. Bei reellen Wurzehn wird sich dieses leicht entscheiden, und bei imaginären kamn man auch immer direct die Factoren der gegebenen Gleichung selhst bestimmen, da $v$ bei diesen immer positiv ist.

Ueberhaupt ist diese Ungewifsheit über das Zeichen von $v$ von keinem practischen Nachtheil. Denn der Fall, wo man bei reellen Wurzeln es vorziehen mufs, die trinomischen Factoren statt der einzelnen Wurzeln selbst zu bestimmen, tritt nur ein, wenn zwei Wurzeln gleich, oder so nahe einander gleich sind, dals sie erst sehr spät sich von einander trennen lassen würden. Unter gleichen Wurzeln werden alle die verstanden, welche der absoluten Gröfse nach ohne Rücksicht auf das Zeichen einander gleich sind oder nahe kommen. Ein solcher Fall ist an sich schon sehr selten; wenn er aber eintritt, so giebt es ein fast immer unfehlbares Kennzeichen, das oder die $v$, die zu reellen Wurzeln gehören, von denen zu unterscheiden, die von imaginären gebildet werden. Denn da alle reellen Wurzeln gleich in der ersten abgeleiteten Gleichung nur positive Wurzeln geben, so kann irgend ein negatives Zeichen überhaupt nur dann in irgend einer der abgeleiteten Gleichungen vorkommen, wenn die erste Gleichung imaginäre Wurzeln hat; und bei den verschiedenen Stufen, die $m$ und mit ihm $\cos m \varphi$ durchgeht, wird auch fast immer in einer der abgeleiteten Gleichungen in diesen Falle einmal ein Minuszeichen erscheinen. Dieser Zeichenwechsel wird einem oder mehreren Coefficienten, in denen er zuerst sich gezeigt hat, wiederum in den meisten Fällen eigen bleiben, und kann besonders bei den höheren Potenzen der Wurzeln als ein sicheres Kennzeichen angesehen werden, dafs der Coefficient, welcher zuerst nach einem solchen Minuszeichen eine bestimmte Grenze erreicht, 
den Modul einer imaginären Wurzel enthält. Man kann daher mit völliger Sicherheit schliefsen, dafs wenn vor einem Gliede, wodurch ein $v$ bestimmt wird, ein anderes vorhergeht, welches bei den höheren Potenzen irgend einmal einen Zeichenwechsel dargeboten hat, das aus diesem Gliede erhaltene $v$ nothwendig posiliv ist, und wird nur bei den $v$, denen nie ein Zeichenwechsel vorangegangen ist, über das Zeichen ungewifs sein können, und also auch nur in diesen seltenen Falle zu dem vorgeschlagenen Mittel zu greifen brauchen.

Endlich wird es in jedem Falle zweckmärsig sein, die beiden linearen Gleichungen in Bezug auf $f$, die aus dem Coefficienten $a_{1}$ und $a_{2 n-1}$ hervorgehen, mit zu benutzen, so dafs man, vermittelst der zuletzt abgeleiteten Gleichungen, immer zwei $f$ weniger als überhaupt erforderlich sind, bestimmt.

IIat man auf diese Weise die erste Näherung für die Werthe von $t$ und $v$, ebenfalls vermittelst der Logarithmen von 5 Decimalen erhalten, so ist es eben so leicht wie bei den reellen Wurzeln, die genaueren Werthe zu finden. Die Gleichung

$$
f x=f x_{0}+\frac{d f x_{0}}{d x_{0}} \Delta x_{0}=\left[x_{0}^{n}\right]+\left[n x_{0}^{n}\right] \frac{\Delta x_{0}}{x_{0}}
$$

findet auch hier statt. Hat der trinomische Factor imaginäre Wurzeln, wo folglich

$$
x_{0}=-g_{0}\left(\cos \varphi_{0}+\sin \varphi_{0} \checkmark-1\right), \quad x_{0}^{\prime}=-g_{0}\left(\cos \varphi_{1}-\sin \varphi_{1} r-1\right),
$$

so erbält man durch Substitution von $\left(-g_{0}\right)^{n} \cos n \varphi_{1}$ für jedes $x_{1 !}^{n}$, und $\left(-g_{i}\right)^{n} \sin n \varphi_{1}$ für jedes $x_{0}^{n}$, den Werth von

und

$$
f x_{0}=\left[\left(-g_{0}\right)^{n} \cos n \varphi_{0}\right]+\left[\left(-g_{0}\right)^{n} \sin n \varphi_{1,}\right] \curlyvee-1
$$

$$
f x_{0}^{\prime}=\left[\left(-g_{0}\right)^{n} \cos n \varphi_{n}\right]-\left[\left(-g_{11}\right)^{n} \sin n \varphi_{0}\right] \curlyvee-1 .
$$

Auf gleiche Weise wird

$$
\left[n x^{n}\right]=\left[n\left(-g_{10}\right)^{n} \cos n \varphi_{11}\right]+\left[n\left(-g_{10}\right)^{n} \sin n \phi_{11}\right] \vee-1
$$

und $\quad\left[n x_{0}^{\prime n}\right]=\left[n\left(-g_{0}\right)^{n} \cos n \varphi_{0}\right]-\left[v\left(-g_{0}\right)^{n} \sin n \varphi_{0}\right] \checkmark-1$.

Endlich wird wegen

$$
\text { Ind } \quad \begin{aligned}
\lg x_{0} & =\lg \left(-g_{0}\right)+\lg \left(\cos \varphi_{0}+\sin \varphi_{0} \checkmark-1\right) \\
& =\lg \left(-g_{0}\right)+\phi_{10} \checkmark-1 \\
\lg x & =\lg (-g)+\phi \checkmark-1 \\
& \text { und eben so } \quad \Delta \lg x_{0}=\Delta \lg x_{0}^{\prime}=\Delta \lg g_{0}-\Delta \varphi_{0} \checkmark-1
\end{aligned}
$$


Setzt man also, was immer erlaubt ist,

$$
\begin{array}{ll}
{\left[\left(-g_{0}\right)^{n} \cos n \varphi_{0}\right]=\boldsymbol{P} \cos \boldsymbol{Q}} & {\left[n\left(-g_{0}\right)^{n} \cos n \varphi_{1]}\right]=\rho \cos \psi} \\
{\left[\left(-g_{0}\right)^{n} \sin n \varphi_{0}\right]=\boldsymbol{P} \sin \boldsymbol{Q}} & {\left[n\left(-g_{0}\right)^{n} \sin n \varphi_{11}\right]=\rho \sin \psi,}
\end{array}
$$

so hat man die zwei Gleichungen

$0=\boldsymbol{P} \cos Q+\boldsymbol{P} \sin Q \checkmark-1+\{\rho \cos \psi+\rho \sin \psi \sqrt{ }-1\}\left\{\Delta \lg g_{0}+\Delta \varphi_{0} \checkmark-1\right\}$

$0=P \cos Q-P \sin Q \checkmark-1+\{\rho \cos \psi-\rho \sin \psi \checkmark-1\}\left\{\Delta \lg g_{0}-\Delta \varphi_{0} \checkmark-1\right\}$, aus welchen man durch Verbindung vermittelst Addition und Substraction erbält

$$
\begin{aligned}
& 0=\boldsymbol{P} \cos \boldsymbol{Q}+\rho \cos \psi \Delta \lg g_{0}-\rho \sin \psi \Delta \varphi_{0} \\
& 0=\boldsymbol{P} \sin \boldsymbol{Q}+\rho \sin \psi \Delta \lg g_{10}+\rho \cos \psi \Delta \varphi_{0}
\end{aligned}
$$

oder nach gehöriger Elimination

$$
\Delta \log g_{0}=-\frac{P \cos (Q-\psi)}{\rho} M, \quad \Delta \varphi_{0}=-\frac{P \sin (Q-\psi)}{\rho},
$$

wo der Factor $\boldsymbol{M}$ wie oben den Modulus des briggischen Systems bezeichnet. Aus beiden kann man, wenn man es vorzieht, ableiten

oder

$$
\begin{aligned}
& \Delta f_{0}=-\frac{2 P g_{0} \cos \left(Q-\psi+\varphi_{0}\right)}{\rho} \\
& \Delta \lg f_{0}=-\frac{P \cos \left(Q-\psi+\varphi_{0}\right)}{\rho \cos \varphi_{0}} M .
\end{aligned}
$$

Die Rechnung kommt sonach in Wesentlichen auf die Substitution von den beiden Werthen von $x^{n}=(-g)^{n} \cos n \varphi$ und $x^{n}=(-g)^{n} \sin n \varphi$ hinaus, da die Ermittelung von $n(-g)^{n} \cos n \varphi$ und $n(-g)^{n} \sin n \varphi$, oder die Multiplication jedes Gliedes mit dem Exponenten der Potenz von $x$, welche in ihm vorkommt, kaum in Anschlag zu bringen ist.

Sind beide Wurzeln reell, so substituirt man jede einzelne in die Gleichung, und bestimunt ihre Correction, wie oben gezeigt ward, oder wenn man es vorzieht, so kann man die Correction von $v$ und $t$ suchen. Die Substitution der Wurzeln giebt, wenn $\sqrt{ } \boldsymbol{v}=g$,

$$
\left[\left(-g_{0} y_{0}\right)^{n}\right]=\boldsymbol{A}, \quad\left[\left(-g_{0} y_{0}^{-1}\right)^{n}\right]=\boldsymbol{B},
$$

weil die beiden Werthe von $x$ sind $-g_{0} y_{0}$ und $-\frac{g_{0}}{y}$, und daraus folgen die Differentialquotienten in Bezug auf den Logarithmen der Wurzeln Man bat folglich

$$
\left[n\left(-g_{11} y_{0}\right)^{n}\right]=p, \quad\left[n\left(-g_{0} y_{0}^{-1}\right)^{n}\right]=q .
$$

woraus

$$
\begin{aligned}
& 0=A+p\left\{\Delta \lg g_{0}+\Delta \lg y_{0}\right\} \\
& 0=B+q\left\{\Delta \lg g_{0}-\Delta \lg y_{0}\right\},
\end{aligned}
$$


8. Encke, über allgemeine Auflösung der numerischen Gleichungen.

$$
\begin{aligned}
& \frac{1}{M} \Delta \lg g_{0}=-\frac{1}{2}\left\{\frac{A}{p}+\frac{B}{q}\right\} \\
& \frac{1}{M I} \Delta \lg y_{0}=-\frac{1}{2}\left\{\frac{A}{p}-\frac{B}{q}\right\}
\end{aligned}
$$

und dann wegen $\Delta \lg t_{0}=\Delta \lg g_{0}+\Delta \lg \left(y_{0}+\frac{1}{y_{0}}\right)$ für Wurzeln, die gleiche Zeichen haben, folgen wird:

$$
\begin{aligned}
\frac{1}{M} \Delta \lg v_{0} & =-\left\{\frac{A}{p}+\frac{B}{q}\right\} \\
\frac{1}{M I} \Delta \lg t_{0} & =-\frac{1}{2}\left(\frac{A}{p}+\frac{B}{q}\right)-\frac{1}{2} \cos \varphi\left\{\frac{A}{p}-\frac{B}{q}\right\} \\
& =-\left\{\frac{A}{p} \cos \frac{1}{2} \phi^{2}+\frac{B}{q} \sin \frac{1}{2} \phi^{2}\right\}
\end{aligned}
$$

und für $\Delta \lg t_{0}=\Delta \lg g_{0}+\Delta \log \left(y_{0}-\frac{1}{y_{0}}\right)$, für Wurzeln, die ungleiche Zeichen haben,

$$
\begin{aligned}
\frac{1}{M} \Delta \lg v_{0} & =-\left\{\frac{A}{p}+\frac{B}{q}\right\} \\
\frac{1}{M} \Delta \lg t_{0} & =-\frac{1}{2}\left\{\frac{A}{p}+\frac{B}{q}\right\}-\frac{1}{2} \sec \varphi\left\{\frac{A}{p}-\frac{B}{q}\right\} \\
& =-\frac{1}{\cos \varphi}\left\{\frac{A}{p} \cos \frac{1}{2} \phi^{2}-\frac{B}{q} \sin \frac{1}{2} \phi^{2}\right\} .
\end{aligned}
$$

In diesem letzten Satze ist durch die Ermittelung der trinomischen Factoren die Auflösung der Aufgabe vollständig enthalten, und es wird nun keine Schwierigkeit haben, in dem allgemeinen Falle, wo imaginäre und reelle Wurzeln zugleich vorkommen, den Gang der Operationen und die Form des Endresultats zu übersehen. Man kann dazu entweder die beiden oben gegebenen Formen für reelle $W$ urzeln und für imaginäre mit einander multipliciren, wodurch man erhalten wird

$$
\begin{aligned}
& \boldsymbol{x}^{2 n}+\left\{\left[\boldsymbol{a}^{m}\right]+\left[\boldsymbol{f}_{m}\right]\right\} \boldsymbol{x}^{2 n-1}+\left\{\left[\boldsymbol{a}^{m} \boldsymbol{b}^{m}\right]+\left[\boldsymbol{a}^{m} \boldsymbol{f}_{m}\right]+\left[\boldsymbol{g}^{2 m}\right]\right\} \boldsymbol{x}^{2 n-2} \\
& +\left\{\left[\boldsymbol{a}^{m} \boldsymbol{b}^{m} \boldsymbol{c}^{m}\right]+\left[\boldsymbol{a}^{m} \boldsymbol{b}^{m} f_{m}\right]+\left[\boldsymbol{a}^{m} \boldsymbol{g}^{2 m}\right]+\left[\boldsymbol{g}^{2 m} \boldsymbol{f}_{m}^{\prime}\right]\right\} \boldsymbol{x}^{2 n-3} \\
& +\left\{\left[\boldsymbol{a}^{m} \boldsymbol{b}^{m} \boldsymbol{c}^{m} \boldsymbol{d}^{m}\right]+\left[\boldsymbol{a}^{m} \boldsymbol{b}^{m} \boldsymbol{c}^{m} \boldsymbol{f}_{m}\right]+\left[\boldsymbol{a}^{m} \boldsymbol{b}^{m} \boldsymbol{g}^{2 m}\right]+\left[\boldsymbol{a}^{m} \boldsymbol{g}^{2 m} \boldsymbol{f}_{m}^{\prime}\right]\right. \\
& \left.+\left[\boldsymbol{g}^{2 m} \boldsymbol{g}^{2 m}\right]\right\} \boldsymbol{x}^{2 n-} \ldots . . \text { etc. }=0 .
\end{aligned}
$$

Noch einfacher betrachtet man aber jede Gleichung, nachdem man nöthigenfalls ihren Grad durch Multiplication mit $x$ zu einer geraden Zahl gemacht hat, als ein Product der trinomischen Factoren

$$
\left(x^{2}+t x+v\right)\left(x^{2}+t^{\prime} x+v^{\prime}\right)\left(x^{2}+t^{\prime \prime} x+v^{\prime \prime}\right) \ldots=0 \text {. }
$$

Wenn dann durch Erhebung der Wurzeln zur $m^{\text {ten }}$ Potenz die Form erhalten ist: 


$$
x^{2 n}+C_{1} x^{2 n-1}+C_{2} x^{2 n-2}+C_{3} x^{2 n-3}+C_{4} x^{2 n-4} \ldots .+C_{2 n}=0,
$$

so findet in Allgemeinen, unter der Annahme, dafs $v>v^{\prime}, v^{\prime}>v^{\prime \prime}$, $\boldsymbol{v}^{\prime \prime}>\boldsymbol{v}^{\prime \prime \prime}, \ldots v^{(n-1)}>v^{(n)}$, und jede reelle Wurzel, welche zu einem $v$ gehört, gröfser ist als jede zu einem kleineren $v$ gehörige Wurzel, oder die Quadratwurzel aus einem kleineren $v$, das Verhalten statt, dals nach der Gröfse der $v$ geordnet:

$$
\begin{aligned}
& \boldsymbol{C}_{2}=\boldsymbol{v}^{m} \\
& \boldsymbol{C}_{4}=\boldsymbol{v}^{m} \boldsymbol{v}^{\prime m} \\
& \boldsymbol{C}_{6}=\boldsymbol{v}^{m} \boldsymbol{v}^{\prime m} \boldsymbol{v}^{\prime \prime^{m}} \\
& \vdots \\
& \boldsymbol{C}_{2 r+2}=\boldsymbol{v}^{m} \boldsymbol{v}^{\prime m} \boldsymbol{v}^{\prime \prime^{m}} \ldots \boldsymbol{v}^{\mathrm{r}^{m}} .
\end{aligned}
$$

Die Coefficienten $\boldsymbol{C}_{1}, \boldsymbol{C}_{3}, \boldsymbol{C}_{5}$, überhaupt die von der Form $\boldsymbol{C}_{2 r+1}$ sind dagegen Functionen der verschiedenen $t$; wenn irgend ein $\boldsymbol{v}^{(r)}$ zu einem trinomischen Factor gehört, dessen Wurzeln reell siud, so wird $\boldsymbol{C}_{2 r+1}$ die Form haben

$$
C_{2 r+1}=v^{m} v^{\prime^{\prime m}} \ldots v^{(r-1)^{m}} a_{r}^{m}
$$

wo $a_{r}$ die gröfste reelle Wurzel ist, die $\mathrm{zu} \boldsymbol{v}_{r}$ gehört; das letztere hat dann den Werth $a_{r} b_{r}$ und man findet durch Division

$$
\frac{C_{2 r+1}}{C_{2 r}}=a_{r}^{m}, \quad \frac{C_{2 r+2}}{C_{2 r+1}}=b_{r}^{m} \text {. }
$$

Wenn dagegen $v^{(r)}$ zu einem trinomischen Factor gehört, dessen Wurzelı imaginär sind, so nähert sich $\boldsymbol{C}_{2 r+1}$ nicht continuirlich einer bestimmten Grenze, sondern hat stets Werthe, die niemals den Werth von

$$
2 v^{m} v^{\prime m} v^{\prime / m} \ldots v^{(r-1)^{m}} v^{(r)^{\frac{1}{2} m}}
$$

überschreiten können. Der Coefficient $\boldsymbol{C}_{2 r+1}$ behält folglich immer schwankende, häufig mit dem Minuszeichen behaftete Werthe, welches letztere, wenn es sich in irgend einer der abgeleiteten Gleichungen zeigt, ein unfehlbares Kennzeichen ist, dafs die gegebene Gleichung imaginäre Wurzeln hat. Hat man durch Division von

$$
\frac{C_{2 r+2}}{C_{2 r}}=v^{r}
$$

die $v^{r}$ gefunden, sie mögen zu imaginären Wurzelı gehören oder zu reellen, so benutzt man zur Bestimmung der $t$, wenn nicht mehr als 4 imagiuäre Wurzelu vorhanden sind oder zwei $t$ gesucht werden, die linearen Gleichungen, die sich für die $t$ aus den Coefficienten $\alpha_{1}$ und $\alpha_{2 n-1}$ der gegebenen Gleichung finden. Werden mehrere $t$ verlangt, so substituirt man 
die Werthe der verschiedenen $v$ in die oben entwickelten Gleichungen vom $n^{\text {ten }}$ und $(n-1)^{\text {ten }}$ Grade, und erhält durch die Aufsuchung ihres gemeinschaftlichen Divisors den Werth des zu jedem $v$ gehörigen $t$.

Im Falle endlich, dafs ein $v^{(r)}$ zu völlig gleichen Wurzeln gehört, so hat der Coefficient $\boldsymbol{C}_{2 r+1}$ den Werth

$$
\boldsymbol{C}_{2 r+1}=2 \boldsymbol{v}^{m} \boldsymbol{v}^{\prime m} \ldots \boldsymbol{v}^{r-1)^{m}} \boldsymbol{v}^{(r)^{\frac{3}{2} m}}
$$

denselben, welcher bei imaginären Wurzeln die äufserste Grenze bildet.

Ausnahmen von dieser allgemeinen Regel treten nur ein, wenn die obigen Bedingungen nicht alle erfült sind, wenn also z. B. gleiche reelle Wurzeln oder der Modul eines imaginären Wurzelpaares zwischen zwei oder mehreren reellen Wurzeln liegt, die der Grölse der $v$ nach zu einem und demselben $v$ gehören. In diesem Falle ordnen sich die Glieder, die zu solchen Ausnahmen gehören, immer so, wie die Reihefolge der Coefficienten in den einfachen Factoren, welche diese Ausnahme bilden, sich darstellt. Wenn also 3 gleiche reelle Wurzeln vorhanden wären, die mit der nächststehenden ungleichen reellen Wurzel ein Product zweier trinomischer Factoren bilden werden, so ist wegen $(x+a)^{3}(x+b)=x^{4}+(3 a+b) x^{3}+\left(3 a^{2}+3 a b\right) x^{2}+\left(3 a^{2} b+a^{3}\right) x+a^{3} b$, für $b<a$ die Aufeinanderfolge der $C$

$$
\begin{aligned}
& \boldsymbol{C}_{2 r}=\boldsymbol{v}^{m} \boldsymbol{v}^{\prime m} \ldots \boldsymbol{v}^{(r-1)^{m}} \\
& \boldsymbol{C}_{r+1}=3 \boldsymbol{v}^{\prime n} \boldsymbol{v}^{\prime m} \ldots \boldsymbol{v}^{(r-1)^{m}} a^{m} \\
& \boldsymbol{C}_{2 r+2}=3 \boldsymbol{v}^{m} \boldsymbol{v}^{\prime m} \ldots \boldsymbol{v}^{(r-1)^{m}} \boldsymbol{a}^{2 m} \\
& \boldsymbol{C}_{2 r+3}=\boldsymbol{v}^{m} \boldsymbol{v}^{\prime m} \ldots \boldsymbol{v}^{(r-1)^{m}} \boldsymbol{a}^{3 m} \\
& \boldsymbol{C}_{2++}=\boldsymbol{v}^{m} \boldsymbol{v}^{\prime m} \ldots \boldsymbol{v}^{(r-1)^{m}} \boldsymbol{a}^{3 m} \boldsymbol{b}^{m}=\boldsymbol{v}^{m} \boldsymbol{v}^{\prime m} \ldots \boldsymbol{v}^{(r-1)^{m}} \boldsymbol{v}^{(r)^{m}} \boldsymbol{v}^{(r+1)^{m}} .
\end{aligned}
$$

Und ganz ähnlich werden die übrigen Coefficienten der verschiedenen Potenzen eines Binoms sich in Fällen von mehreren gleichen $W$ urzeln zeigen. Ebenso übersielit man leicht die Reihenfolge, wenn $b>a$ wäre.

Wenn eine reelle Wurzel gleich dem Modul einer imaginären wäre, und also wiederum mit der nächstangrenzenden reellen $W$ urzel verbunden, ein Coppelter trinomischer Factor sich bildete, so geht die Form

$$
\left(x^{2}+f x+g^{2}\right)(x+g)(x+a)
$$

$=x^{4}+(a+f+g) x^{3}+\left(a g+a f+f g+g^{2}\right) x^{2}+\left(a f g+a g^{2}+g^{3}\right) x+a g^{3}$, im Falle $a<g$ wäre, weil $f_{m}$ sich dem $2 g^{m}$ möglicherweise nähern, und auf keinen Fall für immer dagegen als verschwindend betrachtet werden kann, über in: 


$$
x^{4}+* x^{3}+* x^{2}+g^{3 m} x+a^{m} g^{3 m}
$$

und folglich wird die Reihefolge der Coefficienten werden

$$
\begin{aligned}
& \boldsymbol{C}_{2 r}=\boldsymbol{v}^{m} \boldsymbol{v}^{\prime m} \ldots \boldsymbol{v}^{(r-1)^{m}} \\
& \boldsymbol{C}_{2 r+1}=\text { unbestimmt und schwankend } \\
& \boldsymbol{C}_{2 r+2}=\text { unbestimmt und schwankend } \\
& \boldsymbol{C}_{2 r+3}=\boldsymbol{v}^{m} \boldsymbol{v}^{\prime m} \ldots \boldsymbol{v}^{(r-1)^{m}} \boldsymbol{g}^{(r)^{3 m}} \\
& \boldsymbol{C}_{2 r+4}=\boldsymbol{v}^{m} \boldsymbol{v}^{\prime m} \ldots \boldsymbol{v}^{(r-1)^{m}} \boldsymbol{g}^{\left(r^{3 / m}\right.} \boldsymbol{a}^{m}=\boldsymbol{v}^{m} \boldsymbol{v}^{\prime m} \ldots \boldsymbol{v}^{(r-1)^{m}} \boldsymbol{v}^{(r)^{m}} \boldsymbol{v}^{(r+1)^{m}}
\end{aligned}
$$

und ganz ähnlich in allen andern Fällen. Gleiche Moduln bei mehreren Paaren imaginärer Wurzeln werden drei aufeinander folgende unbestimmte Glieder geben, wenn zwei Moduln einander gleich sind, 5 aufeinander folgende unbestimmte Glieder, wenn drei Moduln gleich sind u. s. w. Es wird hiernach keine Schwierigkeit haben, sich in allen Fällen den Gang erklären zu können, und die Resultate daraus herzuleiten.

Es mufs hiebei beachtet werden, dafs wenn man immer zu geraden Potenzen erhebt, die Gleichheit der Wurzeln von der absoluten Gröfse ohne Rücksicht auf das Zeiclen zu verstehen ist, und dafs eben deswegen das Zeichen der $v$, welche nicht zu imaginären Wurzeln gehören, zweideutig ist, wenn man auf die ursprüngliche Gleichung zurückgeht. Die Zweideutigkeit hört auf, wenn man die Gleichung auflöst, welche den Quadraten der Wurzeln entspricht. In ihr sind alle $v$ positiv. Dasselbe gilt von den Zeichen der einfacben reellen Wurzeln, über welche durch unmittelbare Substitution oder auf anderm Wege stets zu entscheiden ist.

Diese Ausnahmefälle von gleichen Wurzeln und Moduln erfordern aber aufserdem noch, bei der Verbesserung der zuerstgefundenen Näherungswerthe, eine besondere Berücksichtigung. Denn wenn man nicht aus der Natur der Aufgabe, welche durch eine Gleichung ausgedrückt wird, weifs, dafs in aller Strenge gleiche Wurzel vorhanden sein müssen, so giebt die erste Näherung, wenn sie auf gleiche Wurzeln und Moduln führt, doch nichts anderes zu erkennen, als dafs diese Gleichung innerhalb der Grenzen dieser ersten Näherung stattfindet. Sie kann aber nicht berechtigten, bei der Verbesserung der gefundenen Werthe diese Gleichlieit beizubehalten und vorauszusetzen. Es müssen daher die verbesserten Werthe durch eine Methode gefunden werden, welche über diesen Punct entscheidet, abgesehen davon, dafs die oben angeführten Formeln für die Verbesserung der ersten Werthe, die Ungleichheit sämtlicher Wurzeln 
8. Encke, über allgemeine Auflösung der numerischen Gleichungen.

und selbst eine merkliche Ungleichheit voraussetzen, welche mindestens das Doppelte der etwanigen Verbesserung beträgt, und für die practische Brauchbarkeit noch stärker sein mufs.

Zur Erhaltung einer solchen Verbesserungsmethode setze man wie oben

$$
f x=(x+a)(x+b)(x+c)(x+d) \ldots
$$

und bezeichne aufserdem das Product

$$
\left(1+\frac{1}{x+a} y\right)\left(1+\frac{1}{x+b} y\right)\left(1+\frac{1}{x+c} y\right)\left(1+\frac{1}{x+d} y\right) \ldots
$$

durch die Form

so dals

$$
1+\varepsilon_{1} y+\varepsilon_{2} y^{2}+\varepsilon_{3} y^{3}+\varepsilon_{4} y^{4}+\ldots
$$

$\varepsilon_{1}=\frac{1}{x+a}+\frac{1}{x+b}+\frac{1}{x+c}+\frac{1}{x+d} \cdots \ldots . .=\left[\frac{1}{x+a}\right]$

$\varepsilon_{2}=\frac{1}{(x+a)(x+b)}+\frac{1}{(x+a)(x+c)}+\frac{1}{(x+b)(x+c)}=\left[\frac{1}{(x+a)(x+b)}\right]$

$\varepsilon_{3}=\frac{1}{(x+a)(x+b)(x+c)}+\frac{1}{(x+a)(x+b)(x+d)} \cdots=\left[\frac{1}{(x+a)(x+b)(x+c)}\right]$ u. s. w.

oder $\varepsilon_{m}$ die Summe der Combinationen der $m^{\text {ten }}$ Ordnung ohne Wiederholung vou den $n$ Elementen $\frac{1}{x+a}, \frac{1}{x+b}$, etc. bezeichnet. Man wird nun ohue Mühe finden, dafs jedesmal

$$
\begin{aligned}
\frac{d f x}{d x} & =\varepsilon_{1} f x \\
\frac{1}{1.2} \frac{d^{2} f x}{d} \frac{f x}{x^{2}} & =\varepsilon_{2} f x \\
\frac{1}{1.2 .3} \frac{d^{3} f x}{d \frac{x^{3}}{2}} & =\varepsilon_{3} f x \\
\vdots & \\
\frac{1}{1.2 .3 \ldots m} \frac{d^{m} f x}{d x^{m}} & =\varepsilon_{m} f x
\end{aligned}
$$

sein wird. Es enthält nämlich $\varepsilon_{m} f x$ die Combinationen der $(n-m)^{\text {ten }}$ Ordnung von $n$ Elementen $(x+a),(x+b)$, deren es

$$
\frac{n(n-1)(n-2) \ldots \ldots(n-m+1)}{1.2 .3 \ldots \ldots m}
$$

verschiedene giebt. Jedes einzelne dieser Glieder differentiirt, giebt $(n-\boldsymbol{m})$ Werthe, in welchen immer $(n-m-1)$ solcher Elemente verbunden sind, zusammen sind also in $\frac{d\left(\varepsilon_{m} f x\right)}{d x}$ 


$$
\frac{n(n-1)(n-2) \ldots .(n-m)}{1.2 .3 \ldots \ldots m}
$$

Glieder, deren jedes $(n-m-1)$ Factoren enthält, und da in dieser Summe nur Combinationen von $\operatorname{der}(n-m-1)^{\text {ten }}$ Ordnung enthalten sein können, und aach alle symmetrisch darin enthalten sein müssen, solcher Combinationen aber nur

$$
\frac{n(n-1)(n-2) \ldots \ldots(n-m)}{1.2 .3 \ldots \ldots(m+1)}
$$

möglich sind, so mufs sich jede Combination $(m+1)$ mal wiederholeu. Oder es ist

$$
\frac{d\left(\varepsilon_{m} f x\right)}{d x}=(m+1) \varepsilon_{m+1} f x,
$$

woraus der allgemeine Ausdruck folgt. Wendet man nun auf $f x$ den Taylorschen Lehrsatz an und setzt man

so wird

$$
x=x^{0}+\Delta x^{0}
$$

$$
f x=f x^{0}+\frac{d f x^{0}}{d x^{0}} \Delta x^{0}+\frac{1}{1.2} \frac{d^{2} f x^{0}}{d x^{0^{2}}} \Delta x^{()^{2}}+\ldots
$$

Bezeichnet man also durch $\varepsilon_{m}^{0}$ die $\varepsilon_{m}$, welche "den Factoren $\left(x^{0}+a\right)$, $\left(x^{0}+b\right),\left(x^{0}+c\right)$ etc. entsprechen, und substituirt, so wird

$$
\left.f x=f x^{0}\left\{1+\varepsilon_{1}^{0} \Delta x^{0}+\varepsilon_{2}^{0} \Delta x^{v^{2}}+\varepsilon_{3}^{0} \Delta x^{0^{3}} \ldots\right\}\right\}
$$

welche Reihe Glied für Glied der Taylorschen Reihe entspricht, so dafs das Resultat aus den $m$ ersten Gliedern dieser Reihe vollkommen identisch ist mit dem Resultat aus den $m$ ersten Gliedern der Taylorschen Reihe. Es soll nun $f x$ Null werden für gewisse Werthe von $x$, also mufs, was auch $x^{0}$ für ein Werth sein mag, da nach der Form von $f x$ die Taylorsche Reihe, wenn sie ganz zu Ende geführt wird, stets den strengen Werth giebt, ein Werth von $\Delta x^{0}$ gefunden werden, der der Gleichung

$$
0=1+\varepsilon_{1}^{0} \Delta x^{0}+\varepsilon_{2}^{0} \Delta x^{\left(0^{2}\right.}+\varepsilon_{3}^{0} \Delta x^{0^{3}} \ldots
$$

entspricht, und jedes Resultat, was aus dieser Form bei einer gewissen Anzahl Glieder gefunden wird, muls eben so aus der gleichen Anzahl Glieder der Taylorschen Reilie hervorgehen.

Sei jetzt $x^{0}$ ein Werth, der einer negativen Wurzel sehr nahe kommt, etwa $x^{0}=-a^{0}$, so wird $\Delta x^{0}=a^{0}-a$ eine kleine Grölse der ersten Ordnung, deren verschiedene Potenzen nur dann in der Gleichung

$$
0=1+\varepsilon_{1}^{0} \Delta x^{0}+\varepsilon_{2}^{0} \Delta x^{()^{2}}+\varepsilon_{3}^{0} \Delta x^{0^{3}} \ldots
$$

das erste Glied ...1 ... aufheben können, wenn sie mit Factoren multipli- 
cirt werden, deren Nenner ebenfalls kleine Grölsen der ersten Ordnung zu den verschiedenen Potenzen von $\Delta x^{0}$ erhoben enthalten, während die Zähler Gröfsen der $0^{\text {ten }}$ Ordnung sind. Ein solcher Factor wird in

$$
\varepsilon_{1}^{0}=\frac{1}{x^{0}+a}+\frac{1}{x^{0}+b}+\ldots .=\frac{1}{a-a^{0}}+\frac{1}{b-a^{0}}+\ldots
$$

nur das erste Glied sein, wenn die übrigen Wurzeln so unterschieden von $a$ sind, dafs $b-a^{0}, c-a^{\prime \prime}$ nicht als kleine Gröfsen der ersten Ordnung betrachtet werden können. In diesem Falle werden auch die folgenden $\varepsilon_{2}^{0}, \varepsilon_{3}^{0} \ldots$ keine Nenner enthalten, welche als Gröfsen der zweiten und dritten etc. Ordnung die Kleinheit von $\Delta x^{\prime \prime^{2}}, \Delta x^{0^{3}} \ldots$ etc. aufheben, und eine nierkliche Gröfse hervorbringen könnten, weil die stattfindenden Combinationen ohne Wiederholung sind. Man findet folglich ohne merklichen Fehler $\Delta x^{(1)}$ aus

oder

$$
0=1+\varepsilon_{1}^{0} \Delta x^{0}=1+\frac{1}{a-a^{0}} \Delta x^{0}
$$

Vermittelst des ersten Differentials von $f x$ wird man daher bei lauter ungleichen Wurzeln und hinlänglicher Näherung an eine derselben, einen genaueren Werth finden, worin die Anwendbarkeit und Beschränkung der Newtonschen Approximationsmethode ausgesprochen ist.

Wenn aber aufser $a$ noch eine zweite $W$ urzel $b$ dem $a^{0}$ so nahe liegt, dafs $b-a^{0}$ ebenfalls eine kleine Gröfse erster Ordnung ist, so wird nicht allein

$$
\varepsilon_{1}^{0}=\frac{1}{a-a^{0}}+\frac{1}{b-a^{0}},
$$

sondern es kommt nun auch in $\varepsilon_{2}^{0}$ ein Glied vor, welches mit $\Delta x^{0^{2}}$ verbunden ebenfalls eine Gröfse $0^{\text {ter }}$ Ordnung giebt, und nicht übergangen werden darf. Nämlich ohne merklichen Fehler wird

Die Gleichung

$$
\varepsilon_{2}^{0}=\frac{1}{\left(a-a^{0}\right)\left(b-a^{0}\right)} \text {. }
$$

zerlegt sich dann in die Factoren $0=\left(1+\frac{\Delta x^{0}}{a-a^{0}}\right)\left(1+\frac{\Delta x^{0}}{b-a^{0}}\right)$.

Oder: Wenn zwei Wurzeln vorhanden sind, die einander sehr nahe sind, so berechnet man $\frac{d f x^{0}}{d x^{0}}$ und $\frac{1}{1.2} \frac{d^{2} f x^{0}}{d x^{0^{2}}}$, und findet aus der Auflösung der quadratischen Gleichung zwei Wurzeln, welche zu dem Nähe- 
rungswerthe - $a^{0}$ hinzugefügt, die wahren Werthe geben, die man sucht. Die folgenden $\varepsilon_{3}^{0}$, $\varepsilon_{4}^{\prime \prime}$ etc. haben hier weiier keinen Einflufs. In der Regel werden, wenn $a^{0}$ und $b^{0}$ reell sind, beide Wurzeln dieser quadratischen Gleichung reell sein; sind sie gleich, so sind die Hauptwurzeln innerhalb der Grenzen der neuen Näherung wiederum als gleich anzunelımen. Sind sie aber imaginär, so hatte die Hauptgleichung zwei imaginäre Wurzeln von der Form $a+\beta \sqrt{ }-1$ und $a-\beta \sqrt{ }-1$. Indessen mufs in diesem Falle $\beta$ eine kleine Gröfse der ersten Ordnung sein, weil in $\varepsilon_{1}$ die beiden Glieder für imaginäre Wurzeln

sich vereinigen in

$$
\frac{1}{x+a+\beta \sqrt{ }-1}+\frac{1}{x+a-\beta \sqrt{-1}}
$$

$$
\frac{2(x+a)}{(x+a)^{2}+\beta^{2}}=\frac{1}{x+a+\frac{\beta^{2}}{x+a}}
$$

und also nur dann eine kleine Gröfse der ersten Ordnung im Nenner geben können, wenn $\beta$ von derselben Orduung wie $a-a^{0}$ ist. Auf die absolute Gröfse von $\frac{d f x^{0}}{d x^{0}}$ und $\frac{d^{2} f x^{0}}{d x^{0^{2}}}$ kommt es dabei nicht an. Es kann der Fall eintreten, dafs $\frac{d f x^{0}}{d x^{0}}=$ Null wird, sowohl bei reellen als imaginären Wurzeln. Es ist aber unmöglich, dafs alle Differentialquotienten, welche nöthig sind, zugleich Null werden, weil in diesem Falle die Aufgabe eine unbestimmte würde.

Hiemit ist der Weg für alle ferneren Fälle angezeigt. Wenn bei der ersten Näherung $m$ nahe gleiche Wurzeln gefunden sind, so geht mau bis zu $\varepsilon_{m}^{0}$ fort oder bis $\mathrm{zu} \frac{d^{m} f x^{0}}{d x^{0^{m}}}$, und löst die Gleichung vom $m^{\text {ten }}$ Grade auf. Diese Auflösung, die niemals in solchen Ausnahmefällen zu vermeiden sein wird, muls immer zum Ziele führen, und wird es um so leichter, als durch den eingeführten Näherungswerth $x^{\prime}$ die $\Delta x^{\prime}$, wenn sie verschieden sind, ein sehr merkliches Verhältnifs zu einander haben müssen, und die Schnelligkeit der Auflösung von diesem Verhältnifs der Wurzeln zu einander abhängt, wie schon oben bemerkt ward. Auch ist es ein Vorzug dieser Auflösungsmethode, dals man von allen Wurzeln sehr genäherte Werthe findet, und folglich über die mögliche Anzahl einander sehr nahe liegender gar nicht in $Z \mathbf{Z w e i f e l}$ sein kank. 
Für die numerische Rechnung ist es bequemer, zu setzen

$$
x=x^{0}+x^{0} \frac{\Delta x^{0}}{x^{0}}
$$

und die Taylorsche Reihe zu schreiben:

$$
f x=f x+x^{0} \frac{d f x^{0}}{d x^{0}} \cdot \frac{\Delta x^{0}}{x^{0}}+\frac{1}{2} x^{0^{2}} \frac{d^{2} f x^{0}}{d x^{0}}\left(\frac{\Delta x^{0}}{x^{0}}\right)^{2}+\ldots
$$

Es werden nämlich die Werthe

$f x=\left[x^{0^{n}}\right], \quad x^{\prime)} \frac{d f x^{0}}{d x^{0}}=\left[n x^{0^{\prime n}}\right], \quad x^{()^{2}} \frac{d^{2} f x^{0}}{d x^{()^{2}}}=\left[n(n-1) x^{()^{n}}\right]$, etc.

aus dem ersten $f x^{0}$ durch einfache Multiplication jedes Gledes mit $n$ (der Potenz von $x^{\prime \prime}$, die darin vorkommt), mit $(n-1)$ u. s. w. gefunden. Der Werth $\frac{\Delta x^{0}}{x^{0}}$, den man findet, wird dann fast immer gleich $\Delta \log x^{0}$ gesetzt werden können, mit Rücksicht auf den Modulus des briggischen Systems.

Dieses Verfahren gilt allgemein für reelle und imaginäre Wurzeln. Indessen ist es vielleicht nicht überflüssig, in Bezug auf die letzteren eine nähere Entwickelung hinzuzufügen. Wenn zwei Paare imaginärer Wurzeln von der Form $\alpha \pm \beta \gamma-1$ und $\alpha^{\prime} \pm \beta^{\prime} \gamma-1$ einander so nahe liegen, dafs die erste Näherung gleiche imaginäre Wurzeln von der Form $\alpha^{\prime \prime} \pm \beta^{\prime \prime} \checkmark-1$ gegeben hat, so braucht hier nur der Fall betrachtet zu werden, wo $\beta^{\prime \prime}$ eine merkliche Grüfse hat, weil der andere, wenn $\beta^{0}$ sehr klein ist, sich aus der Substitution der reellen Wurzel $x^{0}=-\alpha^{0}$ ergeben würde, wie oben bei zwei gleichen reellen Wurzelı angefülırt ist. In diesem Falle wird $\varepsilon_{1}^{0}$ den Werth haben, wenn $x^{\prime \prime}=-\alpha^{0}-\beta^{\prime \prime} \checkmark-1$ substituirt ist:

$$
\frac{1}{\alpha-\alpha^{0}+\left(\beta-\beta^{0}\right) \sqrt{-1}}+\frac{1}{\alpha^{\prime}-\alpha^{0}+\left(\beta^{\prime}-\beta^{0}\right) \sqrt{1}=1},
$$

denn die Verbindung von $-\alpha^{0}-\beta^{0} \checkmark-1$ mit $\alpha-\beta \gamma-1$ und $\alpha^{\prime}-\beta^{\prime} \gamma-1$ wird in dem Nenuer die sehr merklichen Gröfsen $-\left(\beta+\beta^{\prime \prime}\right) \checkmark-1$ und $-\left(\beta^{\prime}+\beta^{\prime \prime}\right) r-1$ einführen, die sich nicht gegenseilig vernichten. Dasselbe wird in $\varepsilon_{2}^{\prime \prime}$ stattfinden, welches den Werth erhält:

$$
\frac{1}{\left(\alpha-\alpha^{0}+\left(\beta-\beta^{0}\right) \sqrt{-1}\right)\left(\alpha^{\prime}-\alpha^{0}+\left(\beta^{\prime}-\beta^{0}\right) \sqrt{-1}\right)} \text {. }
$$

Die spätern Werthe von $\varepsilon_{3}^{0}$, $\varepsilon_{4}^{0}$ haben keinen Einflufs. Man wird also aus der Gleichung

$$
0=f x^{\prime \prime}+x^{\prime \prime} \frac{d / x^{0}}{d x^{0}} \cdot \frac{\Delta x^{0}}{x^{0}}+\frac{x^{\prime \prime 2}}{1.2} \cdot \frac{d^{2} f x^{0}}{d x^{\prime \prime 2}}\left(\frac{\Delta x^{0}}{x^{0}}\right)^{2},
$$

wenn für $x^{\prime \prime} \operatorname{der}$ Werth $-\alpha^{\prime \prime}-\beta^{\prime \prime} \curlyvee-1$ gesetzt ist, die beiden Wurzeln 
für $\Delta x^{0}$ erhalten

$$
\left\{\Delta x^{0}+\alpha-\alpha^{0}+\left(\beta-\beta^{0}\right) r-1\right\}\left\{\Delta x^{0}+\alpha^{\prime}-\alpha^{0}+\left(\beta^{\prime}-\beta^{0}\right) r-1\right\}
$$

und auf dieselbe Weise, wenn $-\alpha^{0}+\beta^{0} \sqrt{-1}$ substituirt ist, die Wurzeln

$$
\left\{\Delta x^{0}+\alpha-\alpha^{0}-\left(\beta-\beta^{\prime \prime}\right) r-1\right\}\left\{\Delta x^{0}+\alpha^{\prime}-\alpha^{0}-\left(\beta^{\prime}-\beta^{\prime \prime}\right) r-1\right\}
$$

und die Verbindung beider Gleichungen vom zweiten Grade in Bezug auf $\Delta x^{0}$, die aus beiden Substitutionen hervorgehen, wird eine Gleichung vom vierten Grade geben, deren vier Wurzeln sein werden

$$
\left\{\Delta x^{0}+\alpha-\alpha^{0} \pm\left(\beta-\beta^{0}\right) r-1\right\}\left\{\Delta x^{0}+\alpha^{\prime}-\alpha^{0} \pm\left(\beta^{\prime}-\beta^{0}\right) r-1\right\} \text {. }
$$

Sind deshalb die $W$ urzeln wirklich ungleich, so mufs man durch unmittelbare Substitution entscheiden, welches Wurzel-Paar zu $\alpha^{0}+\beta^{0} \checkmark-1$, und welches zu $\alpha^{0}-\beta^{0} r-1$ gehört. Ebenso wird man bei drei nahe gleichen Paaren imaginärer Wurzeln auf eine Gleichung vom $6^{\text {ten }}$ Grade geführt, bei vieren auf eine vom achten u. s. w.

Die Form dieser Gleichungen wird sich am leichtesten übersehen Jassen, wenn man setzt

und damit

$$
a^{\prime \prime}=r^{()} \cos \varphi^{\prime \prime} \quad \beta^{\prime \prime}=r^{\prime \prime} \sin \varphi^{0}
$$

$$
\begin{aligned}
& {\left[\left(-\boldsymbol{r}^{\prime \prime}\right)^{n} \cos n \Phi^{\prime}\right] \quad=\boldsymbol{P} \cos \boldsymbol{Q} \quad\left[\left(-\boldsymbol{r}^{\prime}\right)^{n} \sin n \boldsymbol{\varphi}^{\prime}\right] \quad=\boldsymbol{P} \sin \boldsymbol{Q}} \\
& {\left[n\left(-r^{\prime}\right)^{n} \cos n \phi^{\prime \prime}\right]=\rho \cos \psi \quad\left[\left(n\left(-r^{\prime \prime}\right)^{n} \sin n \varphi^{\prime \prime}\right]=\rho \sin \psi\right.} \\
& {\left[n(n-1)\left(-r^{\prime}\right)^{n} \cos n \phi^{\prime \prime}\right]=\rho^{\prime} \cos \psi^{\prime} \quad\left[n(n-1)\left(-r^{0}\right)^{n} \sin n \varphi^{\prime \prime}\right]=\rho^{\prime} \sin \psi^{\prime}} \\
& {\left[n(n-1)(n-2)\left(-r^{\prime \prime}\right)^{n} \cos n \phi^{\prime \prime}\right]=\rho^{\prime \prime} \cos \psi^{\prime \prime}} \\
& {\left[n(n-1)(n-2)\left(-r^{\prime \prime}\right)^{n} \sin n \phi^{\prime \prime}\right]=\rho^{\prime \prime} \sin \psi^{\prime \prime} \text { etc. }}
\end{aligned}
$$

Es wird dann die Gleichung aus der Substitution von $x^{\prime \prime}=-\alpha^{\prime \prime}-\beta^{\prime \prime} \checkmark-1$ :

$$
\begin{aligned}
n & =\boldsymbol{P} \cos \boldsymbol{Q}+\rho \cos \psi \frac{\Delta x^{0}}{x^{0}}+\frac{1}{2} \rho^{\prime} \cos \psi^{\prime}\left(\frac{\Delta x^{0}}{x^{0}}\right)^{2}+\frac{1}{6} \rho^{\prime \prime} \cos \psi^{\prime \prime}\left(\frac{\Delta x^{0}}{x^{0}}\right)^{3} \ldots \\
& +\left\{\boldsymbol{P} \sin \boldsymbol{Q}+\rho \sin \psi \frac{\Delta x^{0}}{x^{0}}+\frac{1}{2} \rho^{\prime} \sin \psi^{\prime}\left(\frac{\Delta x^{0}}{x^{0}}\right)^{2}+\frac{1}{6} \rho^{\prime \prime} \sin \psi^{\prime \prime}\left(\frac{\Delta x^{0}}{x^{0}}\right)^{3} \ldots\right\} r-1
\end{aligned}
$$

oder, wenn man die $r-1$ wegschafft,

$$
\begin{aligned}
0 & =\left\{\boldsymbol{P} \cos \boldsymbol{Q}+\rho \cos \psi \frac{\Delta x^{0}}{x^{0}}+\frac{1}{2} \rho^{\prime} \cos \psi^{\prime}\left(\frac{\Delta x^{0}}{x^{0}}\right)^{2}+\frac{1}{6} \rho^{\prime \prime} \cos \psi^{\prime \prime}\left(\frac{\Delta x^{0}}{x^{0}}\right)^{3} \ldots\right\}^{2} \\
& +\left\{\boldsymbol{P} \sin \boldsymbol{Q}+\rho \sin \psi \frac{\Delta x^{0}}{x^{0}}+\frac{1}{2} \rho^{\prime} \sin \psi^{\prime}\left(\frac{\Delta x^{0}}{x^{0}}\right)^{2}+\frac{1}{6} \rho^{\prime \prime} \sin \psi^{\prime \prime}\left(\frac{\Delta x^{0}}{x^{0}}\right)^{3} \ldots\right\}^{2},
\end{aligned}
$$

welche letztere Gleichung ebenfalls für die Substitution von $x^{0}=-\alpha^{\prime \prime}+\beta^{\prime \prime} \vee-1$ gilt, so dafs man immer nur auf eine Gleichung von demselben Grade mit der Anzahl der imaginären Wurzeln geführt wird.

In den einfacheren Fällen, wo zwei Paare oder drei Paare imaginärer Wurzeln gleich sind, kommt man indessen nocb eiufacher zum Ziel, 
und ohne alle $\mathbf{Z}$ weideutigkeit, wenn man bei der ersten aus der Substitution von $x^{0}=-\alpha^{0}-\beta^{\prime} r-1$ allein bervorgehenden Gleichung stehen bleibt, und diese Gleichung auflöst. Man bekommt dann unmittelbar die Werthe von $\alpha-\alpha^{0}+\left(\beta-\beta^{0}\right) r-1$ und von $\alpha^{\prime}-\alpha^{0}+\left(\beta^{\prime}-\beta^{\prime \prime}\right) r-1$. Diese Gleichungen unterscheiden sich freilich von den gewöhnlichen dadurch, dars ihre Coefficienten imaginäre Gröfsen sind, aber für die Fälle, für welche man Gleichungen direct auflösen kann, also bis zum $4^{\text {ten }}$ Grade, oder bis zu vier Paaren nahe gleicher imaginärer Wurzeln, wird man diese Auflösung fast mit derselben Leichtigkeit wie bei reellen Coefficienten machen. Es mögen hier noch die einfachen Formeln für die Auflösung einer solchen quadratischen Gleichungen mit imaginären Coefficienten folgen.

Die imaginären Coefficienten lassen sich leicht dividiren und multipliciren, wenn sie auf die Form $\lambda \cos \mu+\lambda \sin \mu \sqrt{-1}$ gebracht sind, weil

$$
\frac{\lambda \cos \mu \pm \lambda \sin \mu V-1}{\lambda^{\prime} \cos \mu^{\prime} \pm \lambda^{\prime} \sin \mu^{\prime} \sqrt{ }-1}=\frac{\lambda}{\lambda^{\prime}}\left\{\cos \left(\mu-\mu^{\prime}\right) \pm \sin \left(\mu-\mu^{\prime}\right) \gamma-1\right\} \text {. }
$$

Man bringt also die gegebene Gleichung

$$
0=\boldsymbol{P}(\cos Q+\sin Q \checkmark-1)+\rho(\cos \psi+\sin \psi r-1) \frac{\Delta x^{0}}{x^{0}}
$$

zuerst auf die Form

$$
+\frac{1}{2} \rho^{\prime}\left(\cos \psi^{\prime}+\sin \psi^{\prime} r-1\right)\left(\frac{\Delta x^{0}}{x^{0}}\right)^{2}
$$

woraus

$$
\begin{aligned}
\left(\frac{\Delta x^{0}}{x^{0}}\right)^{2} & +\frac{2 \varrho}{\varrho^{1}}\left(\cos \left(\psi-\psi^{\prime}\right)+\sin \left(\psi-\psi^{\prime}\right) \checkmark-1\right) \frac{\Delta x^{0}}{x^{0}} \\
& +\frac{2 P}{\varrho^{1}}\left(\cos \left(\varphi-\psi^{\prime}\right)+\sin \left(\boldsymbol{Q}-\psi^{\prime}\right) \checkmark-1\right)=0
\end{aligned}
$$

$$
\begin{aligned}
\frac{\Delta x^{0}}{x^{0}} & =-\frac{\rho\left(\cos \left(\psi-\psi^{\prime}\right)+\sin \right.}{\varrho^{\prime}} \frac{\left.\left(\psi-\psi^{\prime}\right) V-1\right)}{} \\
& \pm \sqrt{\rho^{2}\left(\cos 2\left(\psi-\psi^{\prime}\right)+\sin 2\left(\psi-\psi^{\prime}\right) V-1\right)-2 P \varrho^{\prime}\left(\cos \left(Q-\psi^{\prime}\right)+\sin \left(Q-\psi^{\prime}\right) \gamma-1\right)}
\end{aligned}
$$

Setzt man jetzt, was immer erlaubt ist,

so wird

$$
\begin{aligned}
& \delta^{2} \cos 2 \gamma=\rho^{2} \cos 2\left(\psi-\psi^{\prime}\right)-2 \boldsymbol{P} \rho^{\prime} \cos \left(\boldsymbol{Q}-\psi^{\prime}\right) \\
& \delta^{2} \sin 2 \gamma=\rho^{2} \sin 2\left(\psi-\psi^{\prime}\right)-2 \boldsymbol{P} \rho^{\prime} \sin \left(\boldsymbol{Q}-\psi^{\prime}\right),
\end{aligned}
$$

$$
\frac{\Delta x^{0}}{x^{0}}=-\frac{\rho\left(\cos \left(\psi-\psi^{\prime}\right)+\sin \left(\psi-\psi^{\prime}\right) V-1\right)}{\rho} \pm \frac{\delta(\cos \gamma+\sin \gamma \gamma-1)}{\rho^{\prime}} .
$$

Nun ist

$$
\frac{\Delta x^{0}}{x^{0}}=\frac{\alpha-\alpha^{0}+\left(\beta-\beta^{0}\right) \sqrt{ }-1}{\alpha^{0}+\beta^{0} \sqrt{-1}} .
$$

Crelle's Journal d. M. Bd. XXII. Hft. 3. 
Setzt man also

so wird

$$
\begin{aligned}
& \alpha-\alpha^{0}=l \cos L \quad \beta-\beta^{0}=l \sin L \\
& \alpha^{0}=r^{0} \cos \varphi \quad \beta^{0} \quad=r^{0} \sin \varphi^{\prime \prime} \text {, }
\end{aligned}
$$

$$
\frac{\Delta x^{0}}{x^{0}}=\frac{l}{r^{0}} \cos \left(L-\varphi^{0}\right)+\frac{l}{r^{0}} \sin \left(L-\varphi^{0}\right) r-1,
$$

folglich wird man erhalten

$$
\begin{aligned}
\frac{l}{r^{0}} \cos \left(\boldsymbol{L}-\varphi^{\prime}\right) & =-\frac{\varrho}{\varrho^{\prime}} \cos \left(\psi-\psi^{\prime}\right) \pm \frac{\delta}{\varrho^{\prime}} \cos \gamma \\
\frac{l}{r^{0}} \sin \left(\boldsymbol{L}-\varphi^{0}\right) & =-\frac{\varrho}{\varrho^{\prime}} \sin \left(\psi-\psi^{\prime}\right) \pm \frac{\delta}{\varrho^{\prime}} \sin \gamma
\end{aligned}
$$

Wenn man den wahren Werth von $x$ durch

ausdrückt, so wird

$$
-r \cos \varphi-r \sin \varphi r-1
$$

$$
\begin{aligned}
\boldsymbol{r}^{0} \cos \phi^{0}+l \cos \boldsymbol{L} & =\boldsymbol{r} \cos \varphi \\
\boldsymbol{r}^{0} \sin \phi^{0}+l \sin \boldsymbol{L} & =r \sin \varphi \\
\boldsymbol{r}^{0}+l \cos \left(\boldsymbol{L}-\phi^{\prime}\right) & =r \cos \left(\varphi-\varphi^{\prime \prime}\right) \\
l \sin \left(\boldsymbol{L}-\varphi^{\prime}\right) & =r \sin \left(\varphi-\phi^{\prime \prime}\right)
\end{aligned}
$$

oder

so daís

$$
\begin{aligned}
& \frac{r}{r^{0}} \cos \left(\varphi-\varphi^{\prime}\right)=1-\frac{\varrho}{\varrho^{\prime}} \cos \left(\psi-\psi^{\prime}\right) \pm \frac{\delta}{\varrho^{\prime}} \cos \gamma \\
& \frac{r}{r^{0}} \sin \left(\varphi-\Phi^{\prime}\right)=-\frac{\varrho}{\varrho^{\prime}} \sin \left(\psi-\psi^{\prime}\right) \pm \frac{\delta}{\varrho^{\prime}} \sin \gamma,
\end{aligned}
$$

wobei die doppelten Vorzeichen so zu nehmen sind, dafs die obern zusammengehören und auch die untern.

Die sämtlichen Formeln für zwei Paare gleicher imaginärer Wurzeln sind also folgende:

Man bestimmt aus den Substitutionen von $\left(-r^{0}\right)^{n} \cos n \varphi$ für $x^{\iota^{n}}$ und $\left(-\boldsymbol{r}^{0}\right)^{n} \sin n \varphi$ für $x^{0^{n}}$ die Werthe $\boldsymbol{P}, \boldsymbol{Q}, \rho, \psi, \rho^{\prime}, \psi^{\prime}$. Dann setzt man

$$
\begin{aligned}
& \delta^{2} \cos 2 \gamma=\rho^{2} \cos 2\left(\psi-\psi^{\prime}\right)-2 \boldsymbol{P} \rho^{\prime} \cos \left(\boldsymbol{Q}-\psi^{\prime}\right) \\
& \delta^{2} \sin 2 \gamma=\rho^{2} \sin 2\left(\psi-\psi^{\prime}\right)-2 \boldsymbol{P} \rho^{\prime} \sin \left(\boldsymbol{Q}-\psi^{\prime}\right)
\end{aligned}
$$

und hat sofort:

$$
\begin{aligned}
& \frac{r}{r^{\circ}} \cos \left(\varphi-\varphi^{0}\right)=1-\frac{\varrho}{\varrho^{\prime}} \cos \left(\psi-\psi^{\prime}\right) \pm \frac{\delta}{\varrho^{\prime}} \cos \gamma \\
& \frac{r}{r^{0}} \sin \left(\varphi-\varphi^{0}\right)=-\frac{\rho}{\varrho^{\prime}} \sin \left(\psi-\psi^{\prime}\right) \pm \frac{\delta}{\varrho^{\prime}} \sin \gamma
\end{aligned}
$$

Für den ersten Werth kann man bei der Kleinheit von $\varphi-\phi^{0}$ meistentheils $\frac{r}{r^{0}}$ setzen, für den zweiten, weil $\frac{r}{r^{0}}$ nahe eins, den Werth $\emptyset-\emptyset^{\prime \prime}$. 
Man wird dann in den meisten Fällen schreiben können:

$$
\begin{aligned}
\frac{1}{M} \Delta \lg r^{0} & =-\frac{\rho}{\rho^{\prime}} \cos \left(\psi-\psi^{\prime}\right) \pm \frac{\delta}{\rho^{\prime}} \cos \gamma \\
\Delta \varphi^{0} & =-\frac{\rho}{\rho^{\prime}} \sin \left(\psi-\psi^{\prime}\right) \pm \frac{\delta}{\rho^{\prime}} \sin \gamma .
\end{aligned}
$$

In diesen Ausdrücken sind sowohl die für gleiche Wurzeln enthalten, für welche $\boldsymbol{Q}=\psi=\psi^{\prime}=0$, als auch lärst sich aus ihnen die oben gegebene Bestimmung der verbesserten Werthe bei ungleichen Wurzeln herleiten. Es ist wegen des doppelten Vorzeichens \pm gleichgültig, welchen Werth von $\gamma$ man aus $2 \gamma$ wählt, da es freisteht, sowohl $\gamma$ als $180+\gamma$ zu nehmen. Für drei Paare gleicher imaginärer Wurzeln wird die Cardanische Formel sich ebenfalls ganz bequem gebrauchen lassen.

In dem hier Gegebenen ist die Auflösung der Aufgabe vollständig enthalten. Es kann kein Fall vorkommen, der sich nicht in aller Strenge durch die angegebenen Ausdrücke lösen liefse. Ungleiche Wurzeln, reell oder inaginär, trennen sich von selbst, und bei gleichen oder nahe gleichen Wurzeln sind leichte und strenge Nittel gegeben, von einem gemeinschaftlichen Näherungswerthe aus, entweder der völligen Gleichheit der Wurzeln sich zu versichern, oder die einzelnen Wurzeln selbst zu berechnen. Bei einem practischen Gegenstande wird es jetzt noch ein Interesse haben, an einigen Beispielen den Gang der Rechnung kennen zu lernen.

Als erstes Beispiel möge die Gleichung siebenten Grades dienen, wodurch nach Gaufs die Puncte auf einer gegebenen Abscissenlinie bestimmt werden, welche für die mechanische Quadratur das möglichst vortheilhafte Resultat geben, wenn man überhaupt nicht melr als sieben Ordinaten anwenden will. Diese Gleichung heifst

$$
x^{7}-\frac{7}{2} x^{6}+\frac{63}{13} x^{5}-\frac{175}{52} x^{4}+\frac{175}{143} x^{3}-\frac{63}{286} x^{2}+\frac{7}{429} x-\frac{1}{3+32}=0 .
$$

Sucht man hier zuerst die Logarithmen der Coefficienten auf, welchen die Zeichen ebenso wie den Zahlen vorgesetzt werden, so wird die Gleichung: $x^{7}-0,5440680 x^{6}+0,6853971 x^{5}-0,5270347 x^{4}+0,0877020 x^{3}-9,3429745 x^{2}$

$$
+8,2126407 x-6,4644527=0 .
$$

Es ist für die Rechnung angenehmer, keine Logarithmen von Brüchen zu haben, weil man bei der Erhebung zu sehr hohen Potenzen immer dann beachten mufs, ob -10 oder -20 , oder ein anderes Vielfaches von 10 von der Characteristik abgezogen werden mufs. Man lege deshalb zu 30 * 
dem Logarithmen aller Coefficienten ein Vielfaches einer solchen Zahl hinzu, welche die Brüche wegschafft, und zwar zu dem Coefficienten von $x^{n-r}$ das $r$ fache dieser $Z$ ahl, so wird man alle Wurzeln mit einem bestimmten Factor multiplicirt erhalten. Kann man dabei bewirken, dafs $\alpha_{n}=1$ wird $^{6}$ so wird die Rechnung etwas bequemer. Hier wird der Zweck erreicht, wenn man die Vielfachen von $\frac{10-6,4644527}{7}=0,5050 \% 82$ hinzulegt. Die Gleichung wird dann $x^{7}-1,0491462 x^{6}+1,6955535 x^{5}-2,0422693 x^{4}+2,1080148 x^{3}-1,8683655 x^{2}$ $+1,2431099 x-0,0000001=0$.

Bei dem Anfange der Rechnung zeigt sich hier sogleich, dars man mehr als fünf Decimalen bei den ersten Erhebungen zu höhern Potenzen anwenden mufs. Denn es wird z. B.

$$
\begin{aligned}
& \lg a_{2}^{2}=+3,39111 \\
& \lg -2 \alpha_{1} \alpha_{3}=-3,39245 \\
& \lg +2 \alpha_{4}=+2,40904
\end{aligned}
$$

Wenn aber die Differenz zweier Logarithmen so klein wie hier ist, nur 0,00134, so bewirkt nach den Gaufsischen Tafeln die Unsicherheit einer einzigen Einheit in der letzten, fünften Stelle schon einen Fehler von 330 Einheiten in dem Logarithmen der Differenz, so dafs sich das Resultat gar nicht verbürgen lälst. Man wird also die beiden ersten Erhebungen hier mit 7 Decimalstellen ausführen müssen, so wie es überhaupt vortheilhaft ist, die ersten Erhebungen möglichst genau zu machen. Die Fehler in denselben pflanzen sich zu beiden Seiten in vergröfsertem Maafsstabe fort, besonders bei Gleichungen, wie diese, mit lauter reellen Wurzeln, in denen immer Subtractionen vorkommen. Man findet so:

\section{Potenz der Wurzeln $=2^{1}$}

$x^{7}+1,4180048 x^{6}+2,3959870 x^{5}+3,0189949 x^{4}+3,2738401 x^{3}+3,0739348 x^{2}$ $+2,2004297 x+0,0000002=0$.

Potenz der Wurzeln $=2^{2}$

$x^{7}+2,2735725 x^{6}+4,0410890 x^{5}+5,3386202 x^{4}+6,0534451 x^{3}+5,9093643 x^{2}$ $+4,3578860 x+0,0000004=0$.

Von hier an kann man mit fünf Decimalen rechnen, weil sich doch, des eben bemerkten Umstandes wegen, auch bei sieben Decimalen die letzten Stellen nicht verbürgen lassen werden. Die kleinen nöthigen Hülfs- 
8. Encke, über allgemeine Auflösung der numerischen Gleichungen.

mittel, bei den grofsen Characteristiken eine Anzahl Einheiten erst wegzunelimen, und nach gemachter Addition und Subtraction wieder zuzulegen, wird jeder Rechner sich selbst machen. Schon von jetzt an sind die Producte der entfernter stehenden Coefficienten wenig merklich.

Potenz der Wurzeln $=2^{3}$

$x^{7}+4,12268 x^{6}+7,61495 x^{5}+10,36176 x^{4}+11,96640 x^{3}+11,78333 x^{2}$

$+8,71441 x+0,00000=0$.

Potenz der Wurzeln $=2^{4}$

$x^{7}+7,97094 x^{6}+15,03723 x^{5}+20,65592 x^{4}+23,91840 x^{3}+23,56553 x^{2}$

$+17,42882 x+0,00000=0$.

Potenz der Wurzeln $=2^{5}$

$x^{7}+15,81746 x^{6}+30,04231 x^{5}+41,30800 x^{4}+47,83679 x^{3}+47,13106 x^{2}$

$+34,85 \% 64 x+0,00000=0$.

Potenz der Wurzeln $=2^{6}$

$x^{7}+31,61214 x^{6}+60,08366 x^{5}+82,61598 x^{4}+95,67358 x^{3}+94,26212 x^{2}$

$+69,71528 x+0,00000=0$.

Potenz der Wurzeln $=2^{7}$

$x^{7}+63,22365 x^{6}+120,16732 x^{5}+165,23196 x^{4}+191,34716 x^{3}+188,52424 x^{2}$

$+139,43056 x+0,00000=0$.

Hier wird die Rechnung geschlossen. Bei allen andern Coefficienten sind schon die neuen Werthe die reinen Quadrate der früheren, und auch bei $\alpha_{1}$ wird $\alpha_{1}^{2}-2 \alpha_{2}$ nicht mehr von $\alpha_{1}^{2}$ sich unterscheiden. Es sind folglich alle Wurzeln reell, und wenn man die Logarithmen nach einander subtrahirt, so hat man

$$
\begin{array}{ll}
\lg a^{128}=63,22365 & \lg \boldsymbol{a}=\mathbf{0 , 4 9 3 9 3 5} \\
\lg \boldsymbol{b}^{128}=56,94367 & \lg \boldsymbol{b}=0,444872 \\
\lg \boldsymbol{c}^{128}=45,06464 & \lg \boldsymbol{c}=\mathbf{0 , 3 5 2 0 6 7} \\
\lg d^{128}=26,11520 & \lg \boldsymbol{d}=\mathbf{0 , 2 0 4 0 2 5} \\
\lg \boldsymbol{e}^{128}=125,17708-128,0 & \lg \boldsymbol{e}=\mathbf{9 , 9 7 7 9 4 6} \\
\lg f^{128}=78,90632-128,0 & \lg \boldsymbol{f}=\mathbf{9 , 6 1 6 4 5 6} \\
\lg g^{128}=116,56944-256,0 & \lg \boldsymbol{g}=8,910699
\end{array}
$$

Zieht man von diesen Wurzeln den oben hinzugefügten Logarithmen 0,5050782 ab, so hat man die wahren Wurzeln der gegebenen Gleichung 


$$
\begin{aligned}
& \lg a=9,98886 \\
& \lg b=9,93979 \\
& \lg c=9,84699 \\
& \lg d=9,69895 \\
& \lg e=9,47287 \\
& \lg f=9,11138 \\
& \lg g=8,40562
\end{aligned}
$$

Werden die negativen Werthe dieser Wurzeln, um ihre Zeichen kennen zu lernen und sie zu verbessern, in die Gleichung substituirt, so zeigt sich, dafs sie keinen Fehler geben, der auch noch bei einer Rechnung mit sieben Decimalen bemerkt werden könnte. So z. B. findet man für $\lg b=9,9397900$

$$
\begin{array}{rr}
x^{7}=+0,3789047 & 7 x^{7}=+2,6523329 \\
\alpha_{1} x^{6}=-1,5233790 & 6 \alpha_{1} x^{6}=-9,1402740 \\
\alpha_{2} x^{5}=+2,4229648 & 5 \alpha_{2} x^{5}=+12,1148240 \\
\alpha_{3} x^{4}=-1,9328347 & 4 \alpha_{3} x^{4}=-7,7313388 \\
\alpha_{4} x^{3}=+0,8073689 & 3 \alpha_{4} x^{3}=+2,4221067 \\
\alpha_{5} x^{2}=-0,1669377 & 2 \alpha_{5} x^{2}=-0,3338754 \\
\alpha_{6} x=+0,0142047 & \alpha_{6} x= \pm 0,0142047 \\
\alpha_{7}=-0,0002914 & x^{0} \frac{d f x^{0}}{d x^{0}}=-0,0020199 \\
f x^{0}=\overline{+0,0000003} &
\end{array}
$$

Es würde folglich

$$
\frac{1}{M} \Delta \lg x^{0}=\frac{-0,0000003}{-0,0020199},
$$

wenn überhaupt der Werth von $f x^{0}$ verbürgt werden könnte, was hier keinesweges der Fall ist, da 3 die achte bedeutende Ziffer ist in den Zallen, aus denen $f x^{0}$ gebildet wird, während schon die siebente ungewifs sein muls. Will man also hier die Logarithmen der Wurzeln verbessern, so mufs man bei der Substitution Logarithmen von 10 Decimalen anwenden oder unmittelbar substituiren, eine Mühe, die hier unnöthig scheint, da Gaufs die Wurzeln bis auf 16 Decimalen gegeben hat, so dafs man den strengen Werth mit dem gefundenen genäherten vergleichen kann. Die Logarithmen der wahren Wurzeln und die Unterschiede von der eben gefundenen ersten Näherung sind: 
8. Encke, über allgemeine Auflösung der numerischen Gleichungen.

\begin{tabular}{|c|c|c|}
\hline $\lg a=9,98881$ & Unterschied & 0,00005 \\
\hline$b=9,93990$ & $"$ & 0,00011 \\
\hline$c=9,84691$ & " & 0,00008 \\
\hline$d=9,69897$ & " & 0,00002 \\
\hline$e=9,47287$ & " & 0,00000 \\
\hline$f=9,11138$ & $\eta$ & 0,00000 \\
\hline$g=8,40562$ & $\eta$ & 0,00000 \\
\hline
\end{tabular}

Die Wurzeln (nach meiner Benennung) sind alle negativ. Obgleich diese Unterschiede für die erste Näherung sämtlich höchst unbedeutend sind, der grölste $={ }_{400 \sigma}^{1}$ des Ganzen, so sieht man doch, dafs sie im Grunde blofs von dem bemerkten Umstande bei $\alpha_{2}$ in der ersten Potenzirung herrühren. Ich habe dieses Beispiel als das ungünstigste unter den mir bekannten bei der ersten Näherung gewählt, wie überhaupt die Lage von sieben reellen Wurzeln, sämtlich zwischen 9 und 1, bei jeder Methode, wegen der Nothwendigkeit, mit gröfserer Genauigkeit, als man sonst bei den ersten Versuchen zu thun pflegt, zu substituiren, die Lösung erschwert haben würde. Die Vergröfserung der Wurzeln allein würde bei keiner Methode wesentlich zur Erleichterung beigetragen haben. Hätte man übrigens, wozu aber kein Grund vorhanden war, dem oben gefundenen Werthe von $f x^{0}$ trauen wollen, so würde man erhalten haben

$$
\Delta \lg \text { brigg. } x^{0}=+0,000065 \text {, }
$$

wodurch man der Wahrheit näher gekommen wäre. Ein Zeichen, dafs die Uebereinstimmung der ersten Näherung nicht blofs zufällig war.

Als zweites Beispiel kann die höchste Gleichung dienen, welche Fourier in seinem vortrefflichen Werke pag. 111 unter den Beispielen aufführt:

$$
\begin{gathered}
x^{7}-2 x^{5}-3 x^{3}+4 x^{2}-5 x+6=0 \\
\text { Potenz der Wurzeln }=2^{1} \\
x^{7}+4 x^{6}-2 x^{5}-2 x^{4}+29 x^{3}-14 x^{2}-23 x+36=0 \\
\text { Potenz der Wurzeln }=2^{2} \\
x^{7}+20 x^{6}+78 x^{5}+54 x^{4}+589 x^{3}+1386 x^{2}+1537 x+1296=0 ; \\
\text { oder, um von jetzt an Logarithmen zu gebrauchen : } \\
x^{7}+1,30103 x^{6}+1,89209 x^{5}+1,73239 x^{4}+2,77012 x^{3}+3,14176 x^{2} \\
+3,18667 x+3,11261=0 .
\end{gathered}
$$




$$
\begin{gathered}
\text { Potenz der Wurzeln }=2^{3} \\
x^{7}+2,38739 x^{6}+3, \text { \%0\%7 } 4 x^{5}-4,56350 x^{4}+5,58565 x^{3}+5,39859 x^{2} \\
-6,08996 x+6,22522=0 .
\end{gathered}
$$

Die Minuszeichen in der zweiten Gleichung zeigten schon an, dafs imaginäre Wurzeln vorhanden seien. In der letzten Gleichung kann man aus dem Orte der Minuszeichen mit Sicherheit schliefsen, dafs der Coefficient von $x^{3}$ einen Modul, und das bekannte Glied einen zweiten Modul geben wird.

Potenz der Wurzeln $=2^{4}$

$$
\begin{aligned}
x^{7}+4,69313 x^{6}+7,64995 x^{5}-9,39198 x^{4} & +11,18557 x^{3}+11,94810 x^{2} \\
& +11,82750 x+12,45044=0 .
\end{aligned}
$$

Potenz der Wurzeln $=2^{5}$

$x^{7}+9,3 \% 002 x^{6}+15,34998 x^{5}-18,87658 x^{4}+22,44623 x^{3}+23,75387 x^{2}$

$-24,65849 x+24,90088=0$.

Potenz der Wurzeln $=2^{6}$

$x^{7}+18,73971 x^{6}+30,70300 x^{5}-37,83535 x^{4}+44,89718 x^{3}+47,7603 \% x^{2}$

$+49,0688 \% x+49,801 \% 6=0$.

Potenz der Wurzeln $=2^{7}$

$x^{7}+37,47942 x^{6}+61,40601 x^{5}-75,51594 x^{4}+89, ; 9441 x^{3}+95,49582 x^{2}$

$+97,80854 x+99,60352=0$.

Potenz der Wurzeln $=2^{8}$

$x^{7}+74,95884 x^{6}+122,81202 x^{5}-151,32153 x^{4}+179,58882 x^{3}+190,99129 x^{2}$

$+195,21132 x+199,20 \% 04=0$.

Hier schliefst die Rechnung; da alle Coefficienten, mit Ausnahme zweier, zu imaginären Wurzeln gehöriger, welche durch die Minuszeichen angedeutet waren, reine Quadrate sind und im Fortgange der Rechnung keine Veränderung würden erleiden können. Zieht man jeden vorhergehenden Coefficienten von dem folgenden ab, so geben die Verbindungen der Coefficienten von

$$
\begin{array}{lll}
x^{7} \text { und } x^{6} & \lg a^{256}=74,95884 & \lg a=0,292808 \\
x^{6} \text { und } x^{5} & \lg b^{256}=47,85318 & \lg b=0,186927 \\
x^{5} \text { und } x^{4} & \text { unbestimmt } & \\
x^{5} \text { und } x^{3} & \lg g^{512}=56,77680 & \lg g^{2}=0,221785 \\
x^{3} \text { und } x^{2} & \lg c^{256}=11,40247 & \lg c=0,044541 \\
x^{2} \text { und } x & \text { unbestimmt } & \\
x^{2} \text { und } x^{0} & \lg g^{1512}=8,21575 & \lg g^{\prime 2}=0,032093 .
\end{array}
$$


Da hier nur zwei Paare imaginärer Wurzeln sind, so kann man die beiden in Bezug auf $f$ linearen Gleichungen, die sich aus $\alpha_{1}$ und $\alpha_{6}$ ergeben, anwenden. Hiezu ist es aber erforderlich, erst des Zeichens von $a$, b, c sich zu versichern. Eine beiläufige Substitution zeigt, dafs a positiv ist, $b$ negativ und $c$ negativ. Denn es geben z. B. bei $c$, wenn man $\operatorname{Ig} x=-0,044541$ (oder negativ) substituirt, die ungeraden Potenzen $\boldsymbol{x}^{7}$, $x^{5}, x^{3}, x$ den Werth der aus ihnen erhaltenen Summe

$$
=+10,9106
$$

und die geraden Potenzen $x^{4}, x^{2}$ und $x^{1}$ den Werth der aus ihnen erhaltenen Summe $=+10,9106$.

Es mufs folglich ein positiver Werth von $x$ substituirt werden, oder $c$ negativ genommen. Die beiden linearen Gleichungen sind

$$
\begin{gathered}
a+b+c+f+f^{\prime}=\alpha_{1}=0 \\
g^{2} g^{\prime 2}(a b+a c+b c)+a b c g^{2} f^{\prime}+a b c g^{\prime 2} f=a_{6}=-5,
\end{gathered}
$$

welche in Zahlen ausgedrückt werden

$$
\begin{aligned}
f+f^{\prime} & =+0,68341 \\
3,60055 f+5,57264 f^{\prime} & =+1,25927
\end{aligned}
$$

woraus man erhält$$
f=+1,29258 \quad f^{\prime}=-0,60917 .
$$

Die erste Näherung giebt daher die Gleichung als das Product der Factoren

$$
\begin{array}{r}
(x+1,96249)(x-1,53790)(x-1,10800)\left(x^{2}+1,29258 x+1,66642\right) \times \\
\left(x^{2}-0,60917 x+1,07669\right) .
\end{array}
$$

Will man diese Werthe verbessern, z. B. den ersten der beiden trinomischen Factoren, so findet sich für ihn

$$
\lg r^{0}=0,11089 \quad \phi^{\prime}=59^{0} 5 \%^{\prime} 25^{\prime \prime} 3 .
$$

Der leichteren Rechuung wegen nehme mau aber $\varphi^{\prime \prime}$ in runden Zehnern von Secunden, etwa

Man erhält damit

$$
\varphi^{\prime}=59^{\circ} 57^{\prime} 20^{\prime \prime} \text {. }
$$

$$
\begin{aligned}
& -\boldsymbol{r}^{\left({ }^{\prime} 7\right.} \cos 7 \varphi^{\prime \prime}=-3,0148035 \\
& -a_{2} r^{()^{3}} \cos 5 \varphi^{\prime \prime}=+3,5605688 \\
& -a_{1} r^{()^{3}} \cos 3 \phi^{0}=-6,4534218 \\
& +a_{5} r^{()^{2}} \cos 2 \varphi^{\prime \prime}=-3,3238460 \\
& -a_{4 i} r^{\prime \prime} \cos \phi^{\prime \prime}=+3,2310655 \\
& \text { bek. Glied } \ldots . .=+6,0 \\
& {\left[\left(-r^{(1)}\right)^{n} \cos n \Phi^{0}\right]=\overline{+0,0000630}}
\end{aligned}
$$$$
\text { Crelle's Juurnal d. M. Bd. XXII. Hif. } 3 .
$$

$$
\begin{aligned}
& -\tau \boldsymbol{r}^{11^{7}} \cos 7 \varphi^{0}=-21,1036245 \\
& -5 a_{2} \boldsymbol{r}^{\prime \prime} \cos 5 \varphi^{\circ}=+17,8028440 \\
& -3 a_{4} r^{r^{3}} \cos 3 \varphi^{0}=-19,3602654 \\
& +2 a_{5} \boldsymbol{r}^{\left(i^{2}\right.} \cos 2 \varphi^{0}=-6,6476920 \\
& -a_{6} r^{0} \cos \varphi^{\prime \prime}=+3,2315655 \\
& {\left[n\left(-r^{\prime \prime}\right)^{n} \cos n \phi^{\prime}\right]=-\overline{26,0771724}}
\end{aligned}
$$


242 8. Encke, über allgemeine Auflösung der numerischen Gleichungen.

ferner

$$
\begin{aligned}
& -\boldsymbol{r}^{()^{7}} \sin 7 \phi^{0}=-5,1569226 \\
& -\alpha_{2} r^{()^{5}} \sin 5 \phi^{0}=-6,2226992 \\
& -\alpha_{4} r^{()^{2}} \sin 3 \phi^{0}=+0,0150177 \\
& +\alpha_{5} \boldsymbol{r}^{()^{2}} \sin 2 \phi^{0}=+5,7777520 \\
& -a_{6} r^{\prime} \sin \phi^{\prime \prime}=+5,58 \% 2230 \\
& {\left[\left(-r^{\prime \prime}\right)^{n} \sin n \emptyset^{\prime \prime}\right]=+0,0003709}
\end{aligned}
$$

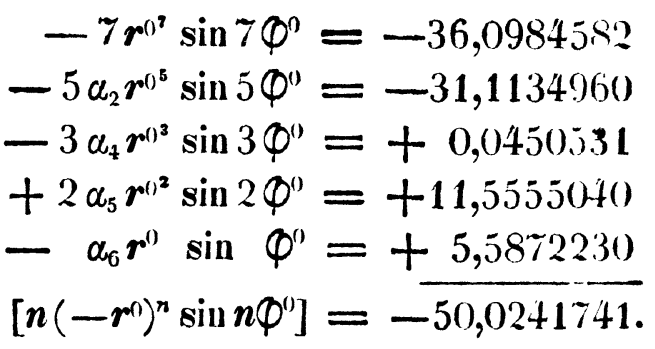

Hieraus folgt dann weiter:

und damit

$$
\begin{aligned}
\lg \boldsymbol{P} & =6,575433 \\
\boldsymbol{Q} & =80^{\circ} 21^{\prime} 35^{\prime \prime}, 7
\end{aligned}
$$

$\lg \rho=1,751380$

$$
\psi=242^{\circ} 28^{\prime} 2^{\prime \prime}, 5
$$

$$
\begin{aligned}
& \Delta \log r^{0}=+0,0000027.50 \quad \Delta \phi^{0}=+0,42 \\
& \text { so dafs } \log g=0,110892 \% .56 \\
& \emptyset=59^{\circ} 57^{\prime} 20^{\prime \prime} 42 \text {. }
\end{aligned}
$$

Auf ähnliche Art wird $g^{\prime}$ und $\varphi^{\prime}$ genauer gefunden und für die reellen Wurzeln hat man z. B. für $a$

$$
\lg a^{0}=0,292808
$$

$$
\begin{aligned}
& -a^{\prime 7}=-112,112997 \\
& -\alpha_{2} a^{\gamma^{5}}=+58,219704 \\
& -\alpha_{4} a^{(2}=+22,6 \% 4894 \\
& -a_{5} a^{(2}=+15,405508 \\
& -a_{4 i} a^{0}=+9,812460
\end{aligned}
$$$$
\text { bek. Glied }=+6,0
$$

$$
\left[\left(-a^{0}\right)^{n}\right]=-\overline{0,000+31,}
$$

$$
\begin{aligned}
-7 a^{()^{3}} & =-784,790979 \\
-5 \alpha_{2} a^{()^{8}} & =+291,098520 \\
-3 \alpha_{4} a^{\prime)^{3}} & =+68,024682 \\
+2 \alpha_{5} a^{()^{2}} & =+30,811016 \\
-\alpha_{6} a^{0} & = \pm \quad 9,812460 \\
{\left[n\left(-{ }^{0}\right) a^{n}\right] } & =-385,044301
\end{aligned}
$$

woraus

$$
\Delta \lg a^{0}=-0,0000005
$$

$$
\lg \boldsymbol{a}=0,2928075 \text {. }
$$

Die Verbesserung jedes eimzelnèn Werthes, isolirt für sich, giebt zuletzt die Factoren der Gleichung:

$$
\begin{gathered}
\left\{x^{2}-0,6092132 x+1,0766801\right\}\left\{x^{2}+1,2926302 x+1,6664238\right\} \times \\
\{x+1,9624901\}\{x-1,5378905\}\{x-1,1080166\},
\end{gathered}
$$

welche, da jeder für sich gefunden ist, die Prüfung der Rechnung gewähreu, dals sein muls:

$$
a+b+c+f+f^{\prime}=\alpha_{1}=0 \text {. }
$$

Die Werthe der ersten Näherung sind in diesem Beispiel so nahe der Wahrheit, wie man es für fünfstellige Logarithmen kaum erwarten durfte. 
8. Encke, über allgemeine Auflösung der numerischen Gleichungen. 243

Als drittes Beispiel einer Gleichung mit mehr als vier imaginären Wurzeln kann die Gleichung dienen

$$
\begin{gathered}
x^{7}+3 x^{4}+6=0 \\
\text { Potenz der Wurzeln }=2^{1} \\
x^{7}+9 x^{4}+36 x^{2}+36=0 . \\
\text { Potens der Wurzeln }=2^{2} \\
x^{7}+81 x^{4}-648 x^{3}+1944 x^{2}-2592 x+1296=0 . \\
\text { Potenz der Wurseln }=2^{3}
\end{gathered}
$$

$x^{7}-1296 x^{5}+11745 x^{4}+104976 x^{3}+629856 x^{2}+1679616 x+1679616=0$. Von hier an Logarithmen:

\section{Potens der Wurzeln $=2^{4}$}

$$
\begin{aligned}
x^{7}+3,41364 x^{6} & +6,27636 x^{5}+8,60926 x^{4}-9,91005 x^{3} \\
& +10,92186 x^{2}+11,84836 x+12,45042=0 .
\end{aligned}
$$

Potenz der Wurzeln $=2^{5}$

$$
\begin{aligned}
x^{7}+6,46828 x^{6} & +12,16012 x^{5}+17,29346 x^{4}+17,89851 x^{3} \\
& +22,31679 x^{2}+22,41671 x+24,90084=0 .
\end{aligned}
$$

Potenz der Wurzeln $=2^{\mathbf{6}}$

$$
\begin{aligned}
x^{7}+12,75961 x^{6} & +23,97079 x^{5}+34,58689 x^{4}-39,91125 x^{3} \\
+ & 44,63668 x^{2}-47,51776 x+49,80168=0 .
\end{aligned}
$$

Potenz der Wurzeln $=2^{7}$

$$
\begin{aligned}
x^{7}+25,49393 x^{6} & +47,63345 x^{5}+69,17378 x^{4}+79,51832 x^{3} \\
& +89,26074 x^{2}+94,72953 x+99,60336=0 . \\
& \text { Polenz der Wurzeln }=2^{8} \\
x^{7}+50,98747 x^{6} & +94,96298 x^{5}+138,34756 x^{4}+158,73567 x^{3} \\
& +178,52144 x^{2}+189,15081 x+199,20672=0 .
\end{aligned}
$$

Hier wird die Rechnung geschlossen, weil die Coefficienten von $x^{6}, x^{4}$, $x^{2}$ und das bekannte Glied reine Quadrate bleiben. Die negativen Coefficienten von $x^{3}$ und $x$ in der $6^{\text {ten }}$ abgeleiteten Gleichung, zeigen, dals die Coefficienten, die auf sie folgen, zwei Moduln bestimmen werden. Auch der Coefficient von $\boldsymbol{x}^{5}$ ist in der dritten abgeleiteten Gleichung negativ. Es ist deshalb selir wahrscheinlich, dafs auch der Coefficient von $x^{4}$ einen 
8. Encke, über allgemeine Auflösung der numerischen Gleichungen.

Modul bestimmt. Die erste Wurzel ist reell. Aus der successiven Division erhält man

$$
\begin{aligned}
& \lg a^{256}=50,98747 \quad \lg a=0,199170 \\
& \lg g^{512}=87,36009 \quad \lg g^{2}=0,341250 \\
& \lg g^{\prime 512}=40,17388 \quad \lg g^{\prime 2}=0,156929 \\
& \lg g^{\prime / 512}=20,68528 \quad \lg g^{1 / 2}=0,080802 \text {. }
\end{aligned}
$$

Un die zu den verschiedenen $g$ gehörigen $f \mathbf{z u}$ finden, wird die Gleichung zu einer vom achten Grade gemacht. Es ist dann $a_{1}=0, \quad a_{2}=0, \quad a_{3}=3, \quad a_{1}=0, \quad a_{5}=0, \quad a_{6}=0, \quad a_{7}=6, \quad a_{3}=0$, womit die $\beta$ und $\gamma$ werden

$$
\begin{aligned}
& \beta=1, \quad \beta_{1}=6 r^{-6}, \quad \beta_{2}=0, \quad \beta_{3}=3, \quad \beta_{4}=0, \\
& \gamma=1, \quad \gamma_{1}=-6 r^{-6}, \quad \gamma_{2}=0, \quad \gamma_{3}=3 \text {. }
\end{aligned}
$$

Die beiden Gleichungen für $t$ werden also

$$
\begin{aligned}
& 0=t^{4}-6 r^{-6} t^{3}-4 r^{2} t^{2}+\left(18 r^{-4}-3\right) t+2 r^{4} \\
& 0=t^{3}+6 r^{-6} t^{2}-2 r^{2} t-\left(6 r^{-4}+3\right) .
\end{aligned}
$$

Wird hier zuerst $\lg r^{2}=0,34125$ substituirt, und die Coefficienten logarithmisch mit vorgesetztem Zeichen geschrieben, so stellt sich die Division beider Gleichungen in einander so :

$$
\begin{aligned}
& 0=t^{4}-9, ; 5440 t^{3}-0,94331 t^{2}+9,86872 t+0,98353 \\
& 0=t^{3}+9,75440 t^{2}-0,64228 t-0,62802 \\
& \lg \boldsymbol{B} \ldots \ldots .0,30103 \quad 0,30103 \quad 0,06969 \\
& -0,05543 t^{3}-0,04228 t^{2}+0,69771 t+0,98353 \\
& 0=t^{3}+0,58685 t^{2}-0,64288 t-0,92810 \\
& \lg \boldsymbol{B}^{\prime} \ldots \ldots 0,06909 \quad \text { o } \quad 0,30198 \\
& -0,51776 t^{2} \ldots \ldots \ldots t+0,62612 \\
& 0=t^{2} \ldots \ldots \ldots t-0,10836 \\
& \lg \boldsymbol{B}^{\prime \prime} \ldots \ldots 0,00000 \quad 0,15026 \\
& +0,58685 t^{2}-0,49202 t-0,92810 \\
& 0=t^{2}-9,9051 \% t-0,34125 \\
& \lg \boldsymbol{B}^{\prime \prime \prime} \ldots \ldots \ldots 0,00000 \quad 0,38189 \\
& +9,90517 t+9,95936 \\
& 0=t+0,05419 \text {. }
\end{aligned}
$$

Die Uebereinstimmung dieses Ietzten Werthes von $\boldsymbol{t}$ mit einer Wurzel der Gleichung $0=t^{2}-0,10836$ zeigt, dals die Annahme eines posi- 
tiven $\boldsymbol{r}^{2}$ richtig ist. Bei der Einfachheit der Coefficienten in den beiden Gleichungen, welche durch $\alpha_{1}=\alpha_{2}=\alpha_{4}=\alpha_{5}=\alpha_{6}=\alpha_{8}=0$ herbeigeführt ist, kann man auch die Gleichungen unmittelbar in einander dividiren, wodurch man erhält :

$$
0=t+\frac{3 r^{12}}{r^{14}+18 r^{4}-36} .
$$

Substituirt man hier $\lg g^{\prime 2}=0,15693$ und $\lg g^{\prime 2}=0,080 \& 0$, so erhält man die logarithmischen Werthe:

$$
0=f^{\prime}+0,28435 \quad 0=f^{\prime \prime}-0,16895 \text {. }
$$

Es sind folglich die Factoren aus der ersten Näherung:

$$
\begin{aligned}
0= & (x+1,58186)\left(x^{2}-1,13289 x+2,19405\right) \\
& \left(x^{2}-1,92464 d x+1,43527\right)\left(x^{2}+1,47553 x+1,20447\right) .
\end{aligned}
$$

Die durch eine Rechnung mit 7 Decimalen gefundenen verbesserten Werthe sind :

$$
\begin{aligned}
0= & (x+1,5818592)\left(x^{2}-1,1328854 x+2,1940798\right) \\
& \left(x^{2}-1,9246556 x+1,4352554\right)\left(x^{2}+1,4756817 x+1,2044862\right) .
\end{aligned}
$$

Als letztes Beispiel endlich einer Trennung gleicher, oder nahe gleicher, reeller oder imaginärer Wurzeln möge die Gleichung dienen:

$$
0=x^{4}+4,002 x^{3}+14,01801 x^{2}+20,03802 x+25,07005 \text {. }
$$

Bei jeder ersten Näherung wird man geneigt sein, die rechle Seite der Gleichung für ein vollständiges Quadrat zu halten. Denn es ist

$$
\begin{aligned}
\left\{x^{2}+2,001 x+5,007\right\}^{2}=x^{4}+4,002 x^{3}+14,018001 x^{2} & +20,038014 x \\
& +25,0 \% 0049
\end{aligned}
$$

welcher Werth sich von der rechten Seite der Gleichung nur in der fünften Decimalstelle erst unterscheidet. Es würde sonach eine sehr weitläufige Rechnung werden, wenn man durch Potenzirung der Wurzeln die einzelnen Wurzeln oder Moduln trennen wohte. Angenommen daher, man babe nach 6 oder 7 Operationen die Ueberzeugung gewonnen, dafs hier zwei einander sehr nahe stehende trinomische Factoren stattinden, so würde man als erste Näherung annehmen

und daraus

$$
g^{2}=g^{\prime 2}=5,007 \quad f=f^{\prime}=2,001
$$

$$
\lg r^{0}=0,3497888 \quad \varphi^{0}=63^{\circ} 26^{\prime} 26^{\prime \prime} 4 \text {. }
$$

Der leichteren Rechnung wegen nehme man

$$
\lg \boldsymbol{r}^{0}=0,3498000 \quad \varphi^{\prime \prime}=63^{\prime \prime} 26^{\prime} 20^{\prime \prime} \text {, }
$$


246 8. Encke, über allgemeine Auflösung der numerischen Gleichungen.

so giebt die Substitution bei Logarithmen von 10 Decimalen, die hier nöthig sind:

$$
\begin{aligned}
& +\boldsymbol{r}^{\prime 1^{4}} \cos 4 \phi^{\circ}=-7,0137170573 \quad 4 \boldsymbol{r}^{0^{4}} \cos 4 \phi^{\circ}=-28,0548682292 \\
& -a_{1} r^{\prime 2} \cos 3 \phi^{0}=+44,1162372122-3 a_{1} \boldsymbol{r}^{()^{2}} \cos 3 \phi^{0}=+132,3487116366 \\
& +a_{2} r^{\prime 2} \cos 2 \phi^{\prime \prime}=-42,1228012449+2 a_{2} r^{\prime 2} \cos 2 \phi^{\prime \prime}=-84,2456024898 \\
& -a_{3} r^{\prime \prime} \cos \phi^{0}=-20,0498010266-a_{3} r^{0} \cos \phi^{\prime \prime}=-20,0498010266 ;
\end{aligned}
$$

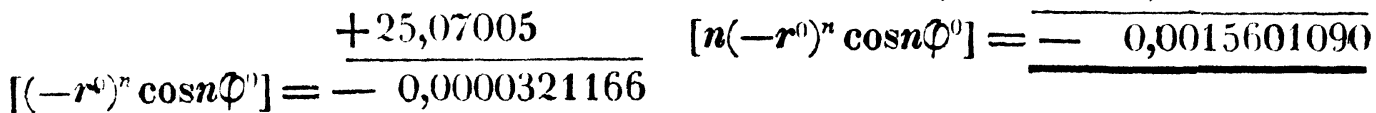

$$
\begin{aligned}
& +r^{r^{4}} \sin 4 \varphi^{\prime \prime}=-24,0 \% 166096 \% 0 \quad 4 r^{(14} \sin 4 \varphi^{\prime \prime}=-96,2866,438680 \\
& -\alpha_{1} \boldsymbol{r}^{r^{3}} \sin 3 \phi^{\prime \prime}=+8,0305364993-3 \alpha_{1} \boldsymbol{r}^{\prime)^{2}} \sin 3 \varphi^{\prime \prime}=+24,09160949 \% 9 \\
& +\alpha_{2} \boldsymbol{r}^{\prime^{2}} \sin 2 \Phi^{\prime \prime}=+56,1476453709+2 \alpha_{2} \boldsymbol{r}^{\prime)^{2}} \sin 2 \phi^{\prime \prime}=+112,2952907418 \\
& -\alpha_{3} r^{(j} \sin \phi^{\prime \prime}=-40,1064968325-\alpha_{3} r^{\prime \prime} \sin \phi^{\prime \prime}=-40,1064968325 \\
& {\left[\left(-r^{\prime \prime}\right)^{n} \sin n \phi^{\prime \prime}\right]=\overline{+0,0000240 \% 07}\left[n\left(-r^{\prime \prime}\right)^{n} \sin n \phi^{\prime \prime}\right]=\overline{-0,0062404608}}
\end{aligned}
$$

Wegen der Gleichlieit der Wurzeln murs hier noch hinzugefügt werden

$$
\begin{aligned}
& 3.4 \boldsymbol{r}^{n^{4}} \cos 4 \phi^{\prime \prime}=-84,1646047 \\
& -2.3 \alpha_{1} \boldsymbol{r}^{(1)^{3}} \cos 3 \phi^{0}=+264,6974233 \\
& +1.2 a_{2} \boldsymbol{r}^{\prime 1^{2}} \cos 2 \phi^{0}=-84,2456025 \\
& \left.\left[n(n-1)\left(-r^{\prime \prime}\right)^{n}\right] \cos n \phi^{\prime \prime}\right]= \pm 96,2872161 \\
& 3.4 r^{\prime \prime} \sin 4 \Phi^{\prime \prime}=-288,8599316 \\
& -2.3 \alpha_{1} r^{(1)^{2}} \sin 3 \phi^{\prime \prime}=+48,1832190 \\
& +1.2 a_{2} r^{\prime 2} \sin 2 \phi^{\prime \prime}=+122,295290 \% \\
& \left.\left[n(n-1)\left(-r^{0}\right)^{n}\right] \sin n \Phi^{\prime}\right]=-128,3814219 .
\end{aligned}
$$

Aus diesen Werthen ergiebt sich

woraus weiter folgt

$$
\begin{array}{ll}
\lg \boldsymbol{P}=5,603531 & \boldsymbol{Q}=143^{\circ} \quad 8^{\prime} 57^{\prime \prime} 0 \\
\lg \rho=7,808380 & \psi=2555749,7 \\
\lg \rho^{\prime}=2,205414 & \psi^{\prime}=3065213,0,
\end{array}
$$

und dann

$$
\lg \delta=9,054660 \quad \gamma=8^{0} 3^{\prime} 29^{\prime \prime}, 35
$$

$$
\begin{aligned}
\frac{r}{r^{\circ}} & =1-0,0000252 \% 6 \pm 0,000699739 \\
\phi-\varphi^{\prime \prime} & =+6,417 \quad \pm 20,434 .
\end{aligned}
$$

Man hat folglich folgende zusammengehörige Werthe

$$
\begin{array}{ll}
\lg g=0,3500928 & \phi=63^{\circ} 26^{\prime} 46^{\prime \prime}, 85 \\
\lg g^{\prime}=0,3494850 & \varphi^{\prime}=63^{\circ} 26^{\prime} 5^{\prime \prime}, 98
\end{array}
$$


und wenn man hieraus die Factoren der Gleichung bildet, so erhält man:

$(x+1,0010021+2,0030005 \checkmark-1)(x+1,0010021-2,0030005 \checkmark-1)$ $(x+0,9999979+2,0000009 \checkmark-1)(x+0,9999979-2,0000009 \checkmark-1)$.

Die Factoren, aus denen sie wirklich gebildet ist, sind

$$
(x+1,1001 \pm 2,003 \sqrt{ }-1)(x+1,000 \pm 2,000 \checkmark-1)
$$

und die kleinen Unterschiede der berechneten Werthe rühren nur davon her, wie die obigen Zahlen ausweisen, dafs auch mit Logarithmen von 10 Decimalen die letzten 3 Decimalstellen in $f x^{\prime)}$ sich nicht mehr verbürgen lassen. Es sind nämlich die wahren Werthe

$$
\begin{array}{ll}
\lg g=0,3500926 & \emptyset=63^{\prime \prime} 26^{\prime} 47^{\prime \prime},() 1 \\
\lg g^{\prime}=0,3444850 & \varphi^{\prime}=63^{\prime \prime} 26^{\prime} \quad 5,42
\end{array}
$$

von welchen die Winkel um 0,"16, die Logarithmen der Moduln um 2 Einheiten der letzten Decimale bei dem ersten abweichend, bei dem zweiten völlig übereinstimmend gefunden sind.

Wollte man die früher erwähnte Auflösung der biquadratischen Gleichung hier anwenden, so wird die cubische Gleichung zur Bestimmung von $y$

$$
y^{3}-14,01801 y^{2}-20,08804396 y+602,6845798014=0,
$$

deren drei reelle $W$ urzeln sind:

$$
(y-10,01401)(y-10,014)(y+6,01)=0,
$$

denn es ist, wenn man sie der Reihe nach mit $a, b, c$ bezeichnet,

$$
\begin{array}{cl}
a+b+c & =-14,01801 \\
a b & =+100,28029614 \\
a c & =-60,18420010 \\
b c & =-60,18414000 \\
a b+a c+b c & =-20,088043960 \\
a b c & =+602,6845798014 .
\end{array}
$$

Der einzige Werth von $y=+10,01401$ macht $\checkmark\left(y^{2}-4 a_{4}\right)$ zu einer reellen Gröfse nämlich $=0,01401$. Es wird folglich

und wegen

$$
\begin{aligned}
& v=\frac{1}{2}\{+10,(01401+0,01401\}=+5,01401 \\
& v^{\prime}=\frac{1}{2}\{+10,(01401-0,01401\}=+5,00000
\end{aligned}
$$

$$
\begin{gathered}
a_{1} y-2 \alpha_{3}=+0,00002802 \\
t=\frac{1}{2}\{4,002+0,(0) 2\}=+2,002 \\
t^{\prime}=\frac{1}{2}\{4,002-0,002\}=+2,000,
\end{gathered}
$$

so dafs die trinomischen Factoren werden 
248 8. Encke, über allgemeine Auflösung der numerischen Gleichungen.

$$
\left\{x^{2}+2,002 x+5,01401\right\}\left\{x^{2}+2 x+5\right\}=0,
$$

wie sie ursprünglich vorausgesetzt waren. Der Werth $y=+10,014$ würde gegeben haben

und der Werth $y=-6,01$

$$
\begin{aligned}
& v=5,00 \tau+0,001 \sqrt{-1} \\
& v^{\prime}=5,00 \tau-0,001 \sqrt{-1} \\
& t=2,001+0,003 \sqrt{-1} \\
& t^{\prime}=2,001-0,003 \sqrt{-1}
\end{aligned}
$$

$$
\begin{aligned}
& v=-3,005+4,005 \checkmark-1 \\
& v^{\prime}=-3,005-4,005 \checkmark-1 \\
& t=+2,001+4,003 \checkmark-1 \\
& t^{\prime}=+2,001-4,003 \checkmark-1
\end{aligned}
$$

